University of Tennessee Health Science Center

UTHSC Digital Commons

$12-2008$

\title{
A Kinematics-Based Testing Protocol to Study the Mechanics of the Human Lumbar Spine
}

Nephi A. Zufelt

University of Tennessee Health Science Center

Follow this and additional works at: https://dc.uthsc.edu/dissertations

Part of the Investigative Techniques Commons

\section{Recommended Citation}

Zufelt, Nephi A. , "A Kinematics-Based Testing Protocol to Study the Mechanics of the Human Lumbar Spine" (2008). Theses and Dissertations (ETD). Paper 365. http://dx.doi.org/10.21007/ etd.cghs.2008.0388.

This Thesis is brought to you for free and open access by the College of Graduate Health Sciences at UTHSC Digital Commons. It has been accepted for inclusion in Theses and Dissertations (ETD) by an authorized administrator of UTHSC Digital Commons. For more information, please contact jwelch30@uthsc.edu. 


\title{
A Kinematics-Based Testing Protocol to Study the Mechanics of the Human Lumbar Spine
}

\begin{abstract}
The objective of this work was to develop a new kinematics-based testing protocol to quantify the axial and shear force components and rotational moment properties of the human cadaveric lumbar motion segment unit (MSU) in response to specific kinematic inputs. Modern, non-fusion spinal devices claim to treat degenerative disc disease better than traditional fusion surgery. Though there have been many biomechanical studies completed on these devices, there is still a debate over their efficacy. Conventional testing methods provide insight into the rotational properties of the MSU but lack the sensitivity or capacity to quantify lumbar MSU's mechanical properties including shear and axial force components. There is a need for a new testing protocol capable of measuring the segmental properties of the lumbar MSU and to study the influence of non-fusion spinal devices on them.

Seven human cadaveric lumbar MSUs were mounted in a custom designed spine robot and flexed or extended about six unique points of rotation located $1 / 4,1 / 2$, and $3 / 4$ of the anterior-posterior depth of the intervertebral disc and at similar points located $5 \mathrm{~mm}$ below the endplate of the subjacent body. The MSUs were rotated until a target bending moment of $8 \mathrm{Nm}$ was reached. Measurements of the shear force along the disc plane, axial force normal to the disc plane, bending moment, and sagittal rotation were used to determine the effects of different prescribed kinematic conditions on MSU mechanics.
\end{abstract}

MSU rotations differed significantly between each rotational point. As the point of rotation moved from anterior to posterior, and from the disc midline to the end plate of the subjacent body, rotation increased. For flexion, significant differences in shear and axial forces occurred between rotational points at $1 / 4$ and $3 / 4$ depth of disc. During extension, shear and axial forces were significantly different between all rotational points along the disc plane.

The results of this study show that the new kinematics-based testing protocol described herein has the capacity to detect significant differences in the segmental rotation and shear and axial forces for small perturbations in the MSU location of the center of rotation for both flexion and extension.

\section{Document Type}

Thesis

Degree Name

Master of Science (MS)

Program

Biomedical Engineering and Imaging

Research Advisor

Denis J. DiAngelo, Ph.D.

\section{Keywords}

MSU mechanics, biomechanical testing, spine robot, kinematics, shear and axial force components

\section{Subject Categories}

Analytical, Diagnostic and Therapeutic Techniques and Equipment | Investigative Techniques | Medicine and Health Sciences 


\title{
A KINEMATICS-BASED TESTING PROTOCOL TO STUDY THE MECHANICS OF THE HUMAN LUMBAR SPINE
}

\author{
A Thesis \\ Presented for \\ The Graduate Studies Council \\ The University of Tennessee \\ Health Science Center
}

\begin{abstract}
In Partial Fulfillment
Of the Requirements for the Degree

Master of Science

In the Joint Graduate Program in Biomedical Engineering and Imaging

From The University of Tennessee

and

The University of Memphis
\end{abstract}

By

Nephi A. Zufelt

December, 2008 
Copyright (C) 2008 by Nephi A. Zufelt

All rights reserved 


\section{Dedication}

This thesis is dedicated to my wonderful wife

Elizabeth Michelle Zufelt

for her support, encouragement, and unceasing optimism and confidence in my ability to accomplish this goal, and to my daughter

Zoe Elizabeth Zufelt

for her beautiful smile that makes every day a good one. 


\section{Acknowledgements}

I would first like to thank my research advisor Dr. Denis DiAngelo for his support of this work. His direction and friendship throughout this experience has been invaluable to me. Dr. Brian Kelly also deserves a space in this section for his extensive guidance on the spine robot and all of the hours that he spent helping me get it up and running. My other graduate committee members, Dr. Yen and Dr. Kasser, have also given feedback and recommendations for my research which has been much appreciated. My lab partner Elizabeth Sander has been a great help throughout. This has been a total team effort and I would not have been able to finish without her hard work.

In the end, all of my academic success has been the collective effort of many different people. My parents Bo and Laura Zufelt instilled in me the importance of getting a good education and they have supported me in my endeavors from the very beginning. There have been many others along the way and I would just like to give a collective thank you to all of them.

Finally, I could not have made it to this point in my career without the support and encouragement of my wife, Elizabeth. She was always there to tell me that I could make it and to remind me to get my studies done. 


\begin{abstract}
The objective of this work was to develop a new kinematics-based testing protocol to quantify the axial and shear force components and rotational moment properties of the human cadaveric lumbar motion segment unit (MSU) in response to specific kinematic inputs. Modern, non-fusion spinal devices claim to treat degenerative disc disease better than traditional fusion surgery. Though there have been many biomechanical studies completed on these devices, there is still a debate over their efficacy. Conventional testing methods provide insight into the rotational properties of the MSU but lack the sensitivity or capacity to quantify lumbar MSU's mechanical properties including shear and axial force components. There is a need for a new testing protocol capable of measuring the segmental properties of the lumbar MSU and to study the influence of non-fusion spinal devices on them.
\end{abstract}

Seven human cadaveric lumbar MSUs were mounted in a custom designed spine robot and flexed or extended about six unique points of rotation located $1 / 4,1 / 2$, and $3 / 4$ of the anterior-posterior depth of the intervertebral disc and at similar points located $5 \mathrm{~mm}$ below the endplate of the subjacent body. The MSUs were rotated until a target bending moment of $8 \mathrm{Nm}$ was reached. Measurements of the shear force along the disc plane, axial force normal to the disc plane, bending moment, and sagittal rotation were used to determine the effects of different prescribed kinematic conditions on MSU mechanics.

MSU rotations differed significantly between each rotational point. As the point of rotation moved from anterior to posterior, and from the disc midline to the end plate of the subjacent body, rotation increased. For flexion, significant differences in shear and axial forces occurred between rotational points at $1 / 4$ and $3 / 4$ depth of disc. During extension, shear and axial forces were significantly different between all rotational points along the disc plane.

The results of this study show that the new kinematics-based testing protocol described herein has the capacity to detect significant differences in the segmental rotation and shear and axial forces for small perturbations in the MSU location of the center of rotation for both flexion and extension. 


\section{Table of Contents}

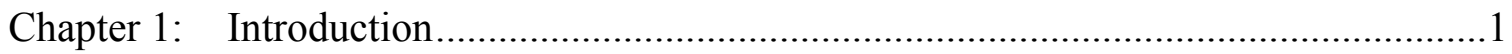

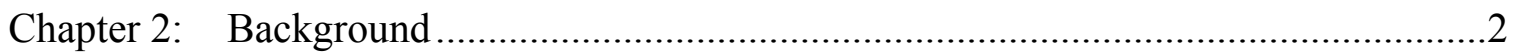

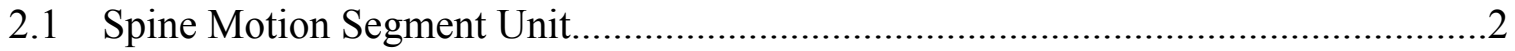

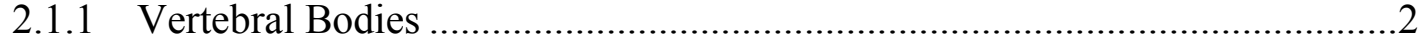

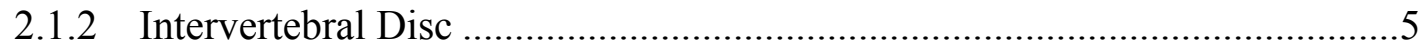

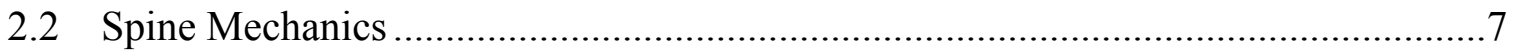

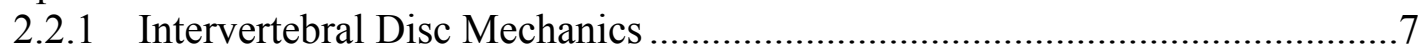

2.2.2 Basic Spine Mechanics ......................................................................

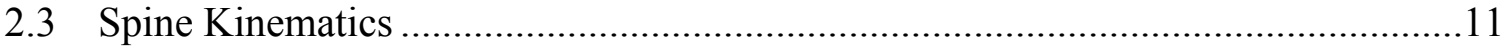

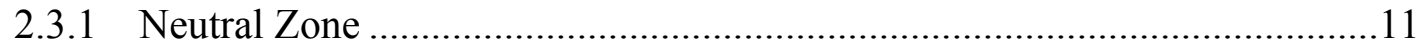

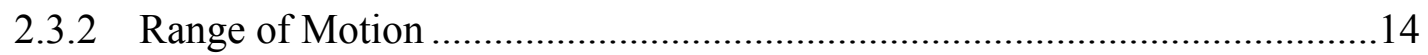

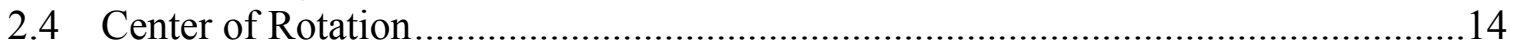

2.4.1 Calculating the Center of Rotation ..........................................................14

2.4.2 Location of the Center of Rotation of the Spine ..........................................16

2.5 Conventional Spine Biomechanical Testing Protocols..............................................17

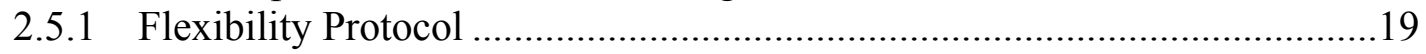

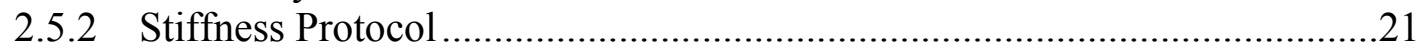

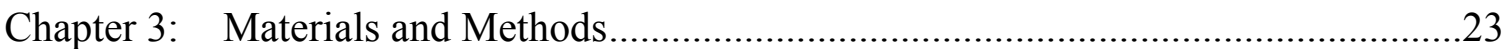

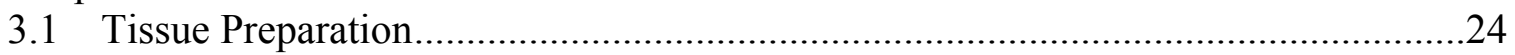

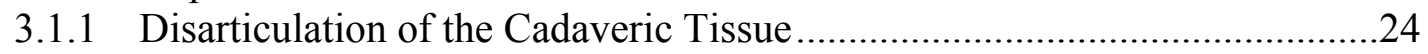

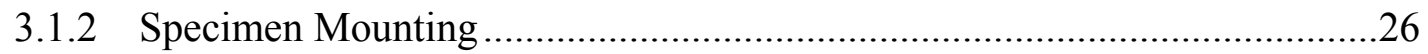

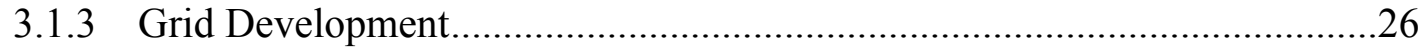

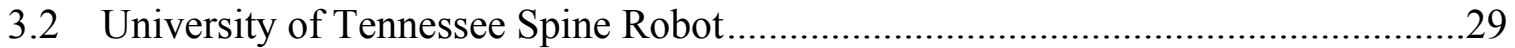

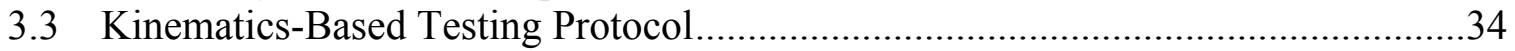

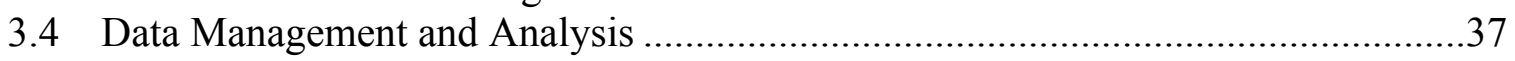

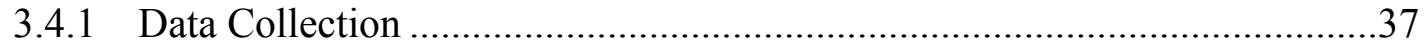

3.4.2 Statistical Analysis...................................................................................

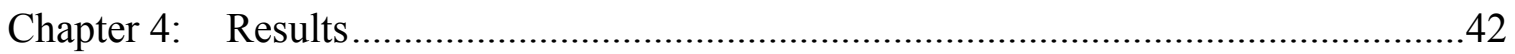

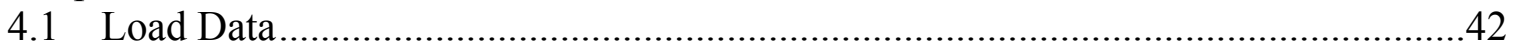

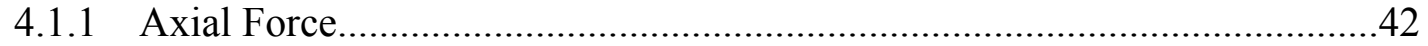

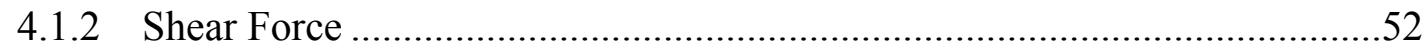

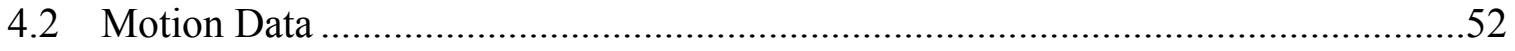

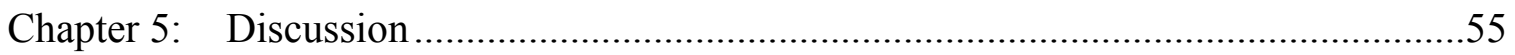

5.1 Matching Load Data to Anatomical and Physiological Interactions ........................55

5.2 Strengths and Limitations of the Kinematics-Based Testing Protocol ......................58

Chapter 6: Conclusion and Recommendations for Future Work....................................60

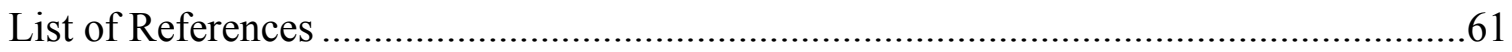


Appendix A: Additional Specimen Grids ...................................................................66

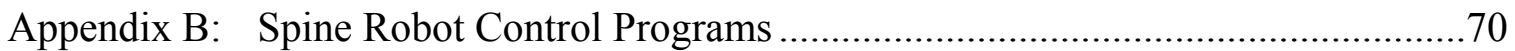

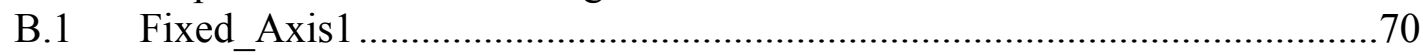

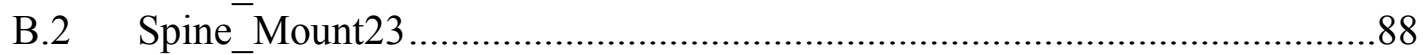

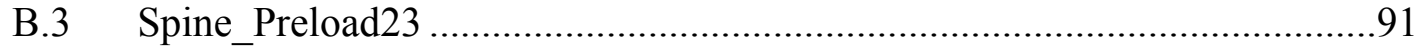

B.4 Write to_File23 ………………………….........................................93

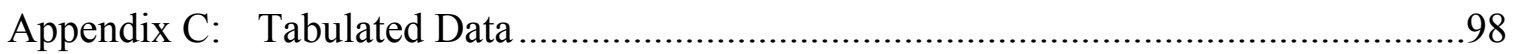

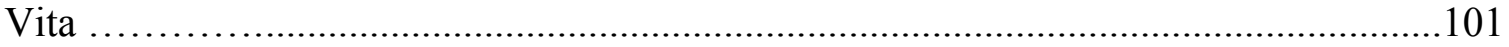




\section{List of Tables}

Table 3.1 $\mathrm{X}$ and $\mathrm{Z}$ Transformation Values for Points A1-P1 .......................................30

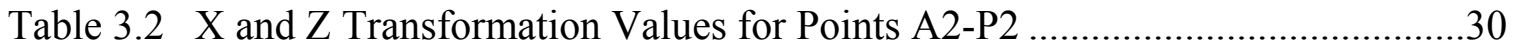

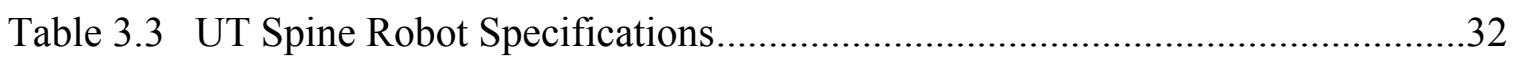

Table 5.1 Resultant Force Vector and Vector Angle for Flexion Rotation .....................57

Table 5.2 Resultant Force Vector and Vector Angle for Extension Rotation..................57

Table C.1 Harvested Spine Condition Flexion Data ....................................................99

Table C.2 Harvested Spine Condition Extension Data................................................ 100 


\section{List of Figures}

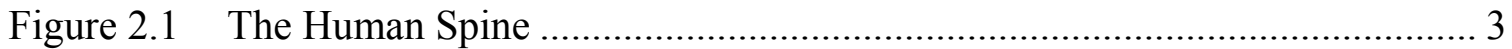

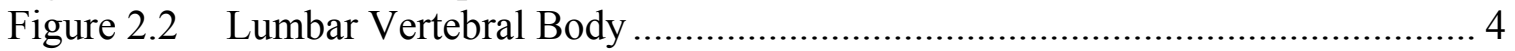

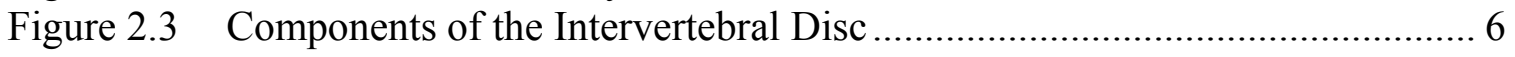

Figure 2.4 Disc Pressure................................................................................. 8

Figure 2.5 Balance of Tension and Compression in the Intervertebral Disc ................ 9

Figure 2.6 Spinal Loading Mechanics.................................................................... 10

Figure 2.7 Degrees of Freedom of Spinal Movement .............................................. 12

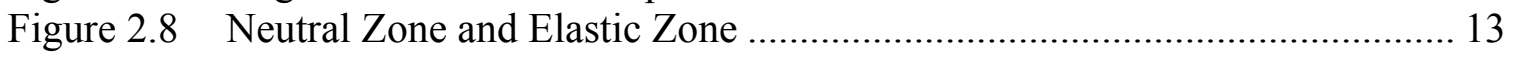

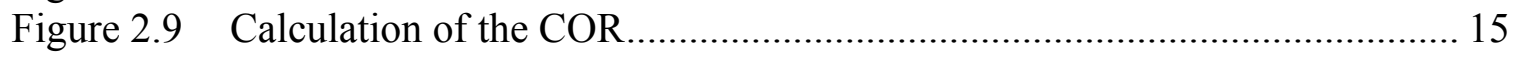

Figure 2.10 Approximate Locations of the Center of Rotation................................... 18

Figure 2.11 Setup to Induce Pure Moment Input ....................................................... 20

Figure 2.12 Setup of Eccentric Load Protocol ............................................................ 22

Figure 3.1 Full Specimen Radiograph Used to Measure Alignment of Segment ......... 25

Figure 3.2 Lumbar Specimen with Caliper Set to $10 \mathrm{~mm}$....................................... 27

Figure 3.3 Lumbar Segment with Point Grid ..................................................... 28

Figure 3.4 UT Spine Robot and Control Cabinet ..................................................... 31

Figure 3.5 Four Degrees of Freedom of the UT Spine Robot.................................... 33

Figure 3.6 Arc of Rotation Prescribed by Robot........................................................ 35

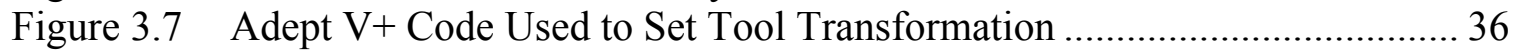

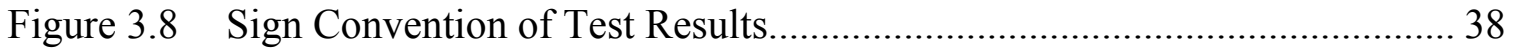

Figure 3.9 Six Axis Load Cell ............................................................................ 40

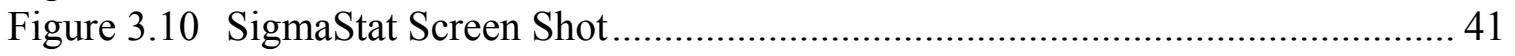

Figure 4.1 Force and Moment versus Displacement Graphs .................................. 43

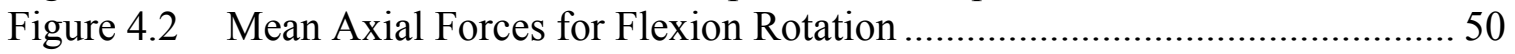

Figure 4.3 Mean Axial Forces for Extension Rotation ........................................... 50

Figure 4.4 Mean Shear Forces for Flexion Rotation ............................................. 51

Figure 4.5 Mean Shear Forces for Extension Rotation ....................................... 51

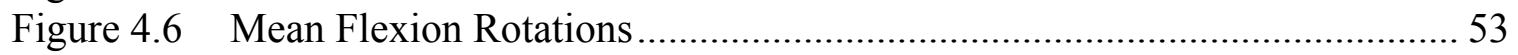

Figure 4.7 Mean Extension Rotations ................................................................. 53

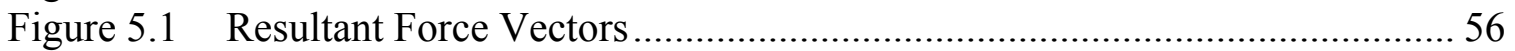

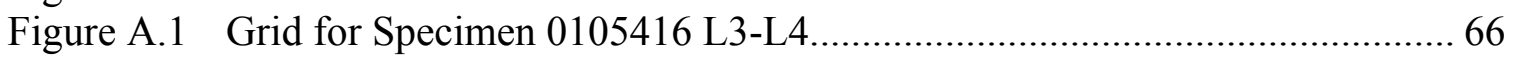

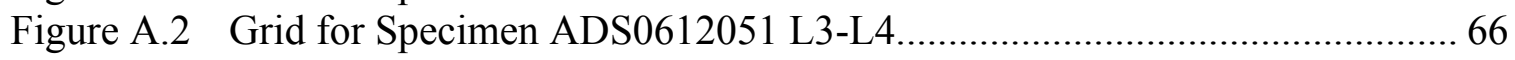

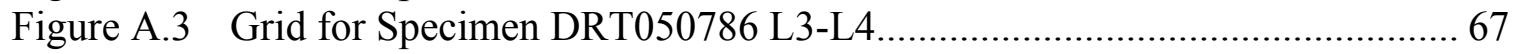

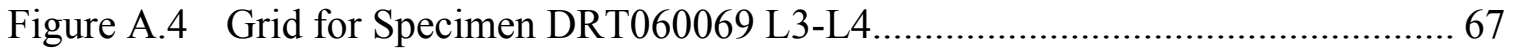

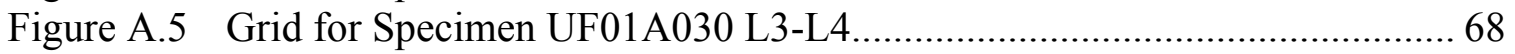

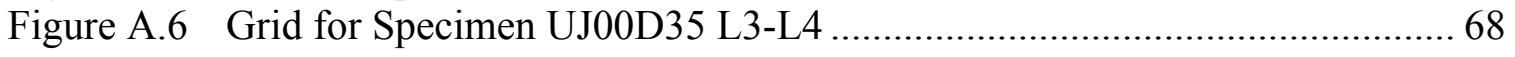

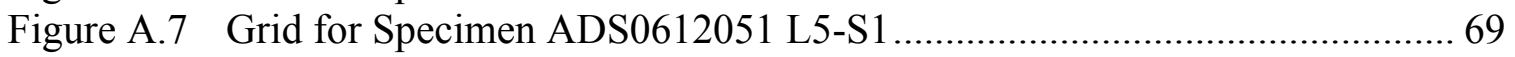




\section{List of Abbreviations}

$\begin{array}{ll}\text { A-P } & \text { Anterior-posterior } \\ \text { ALE } & \text { Adjacent level effect } \\ \text { ANOVA } & \text { Analysis of variance } \\ \text { COR } & \text { Center of rotation } \\ \text { FDA } & \text { Food and Drug Administration } \\ \text { FSU } & \text { Functional spinal unit } \\ \text { IAR } & \text { Instantaneous axis of rotation } \\ \text { ICoR } & \text { Instantaneous center of rotation } \\ \text { MSU } & \text { Motion segment unit } \\ \text { ROM } & \text { Range of motion } \\ \text { TDR } & \text { Total disc replacement } \\ \text { WCS } & \text { World coordinate system }\end{array}$




\section{Chapter 1: Introduction}

Conventional, in-vitro spine biomechanical testing systems were designed to study the effects of spinal fusion surgery on the human lumbar spine by attempting to replicate the physiologic motion of the in-vivo spine. These systems have been used to study many of the spinal devices currently on the market. ${ }^{9 ; 23 ; 47}$ However, due to the challenge of accurately replicating physiologic spine motion, researchers relied on oversimplified testing protocols which provided a standard approach but were not a good representation of what occurs in-vivo. Additionally, these protocols were limited in their ability to measure the basic mechanics of the spine.

In recent years, the trend for spinal surgery has moved away from fusion towards more advanced, non-fusion devices. This includes total disc replacement (i.e. Depuy, Charite ${ }^{\circledR}$; Medtronic, Maverick ${ }^{\mathrm{TM}}$; Synthes, ProDisc L; etc.) and nucleus replacement (i.e. Raymedica, Hydraflex ${ }^{\mathrm{TM}}$; Synthes, Gelifex; Disc Dynamics Inc., Dascor ${ }^{\circledR}$ disc arthroplasty system; etc.) devices, as well as other emerging systems. Furthermore, as these devices evolve, so do the surgical approaches for placing them, which can further impact the surgical outcome. As such, conventional testing protocols previously developed to study spinal fusion instrumentation have limited capacity to characterize the advanced design parameters of non-fusion or compliant devices. ${ }^{25}$ There is a need for testing protocols that provide a better understanding of the effects of these new devices and/or surgical procedures on the resultant segmental kinematics and mechanics. While biomechanical testing protocols have been designed to study some of the properties of the non-fusion devices, ${ }^{16}$ such as the adjacent-level effects (ALE) on the spine, ${ }^{48}$ there has not, to date, been a system or protocol developed that can directly explore the effects of these devices on the mechanics of the spine.

Characteristics of the native spine, such as the inherent center of rotation location, as well as the performance of different spinal devices may be assessed by taking a reverse approach to traditional load application protocols. By imposing a prescribed kinematic input to a specimen and measuring the resultant load response and range of motion, a better understanding of the spinal mechanics and kinematics can be achieved. The objective of this study was to develop a new biomechanical testing protocol designed to study the motion segment unit (MSU) mechanics of the human lumbar spine. 


\section{Chapter 2: Background}

This chapter is divided into five sections. Section one gives a basic overview of important anatomical structures of the lumbar spine in spine kinematics. The second section introduces the basic spine mechanics of the human lumbar motion segment unit. Section three discusses the kinematics of the motion segment unit and some of the critical kinematic parameters used in biomechanical testing. The fourth section is dedicated to explaining the center of rotation and its importance in kinematic testing protocols. Finally, section five discusses the strengths and weaknesses of traditional spine testing protocols.

\subsection{Spine Motion Segment Unit}

Two adjacent vertebral bodies, along with their connecting tissue, form a functional spinal unit (FSU). The FSU has three principal joints responsible for articulations of the spine. The two posterior joints are known as zygapophyseal or facet joints and the anterior joint is the intervertebral disc. The facet joints each consist of two articulating surfaces: those which are located on the inferior vertebrae of the FSU, and the corresponding mating surfaces on the superior vertebrae. Although the facets engage most prominently when the spine is in extension, they allow only small degrees of rotation in flexion, limit axial rotation, and ultimately protect the disc from shear stresses. ${ }^{3}$ These three joints provide the FSU with six degrees of freedom. Functional spinal units are also known as motion segment units (MSU).

\subsubsection{Vertebral Bodies}

The human spine consists of twenty four individual bony structures called vertebral bodies. These bodies are divided into three different regions: cervical (seven vertebral bodies), thoracic (twelve vertebral bodies), and lumbar (five vertebral bodies). The sacrum is also considered to be part of the spinal column and consists of five to nine fused vertebral bodies and a coccyx bone. The cervical and lumbar sections are both naturally lordotic while the thoracic and pelvic sections are naturally kyphotic. The individual lordotic and kyphotic curvature of each section can be seen in the lateral view of Figure 2.1 with the vertebrae groups shown in the posterior view. Each vertebral body has a hard shell of cortical bone of 1-3 mm thickness and an inner cancellous bone structure. A typical lumbar vertebral body is shown in Figure 2.2.

In the axial view of Figure 2.2 the vertebral body is separated into an anterior and posterior section. The anterior section consists of the vertebral body. The superior and inferior surfaces of the vertebral bodies consist of a thin layer of hyaline cartilage that acts as both a support structure and as a source of nutrients for the intervertebral disc (disc). These "endplates" contain fine pores to allow for the diffusion of nutrients from the vascularity of the vertebral bodies to the avascular intervertebral disc. 


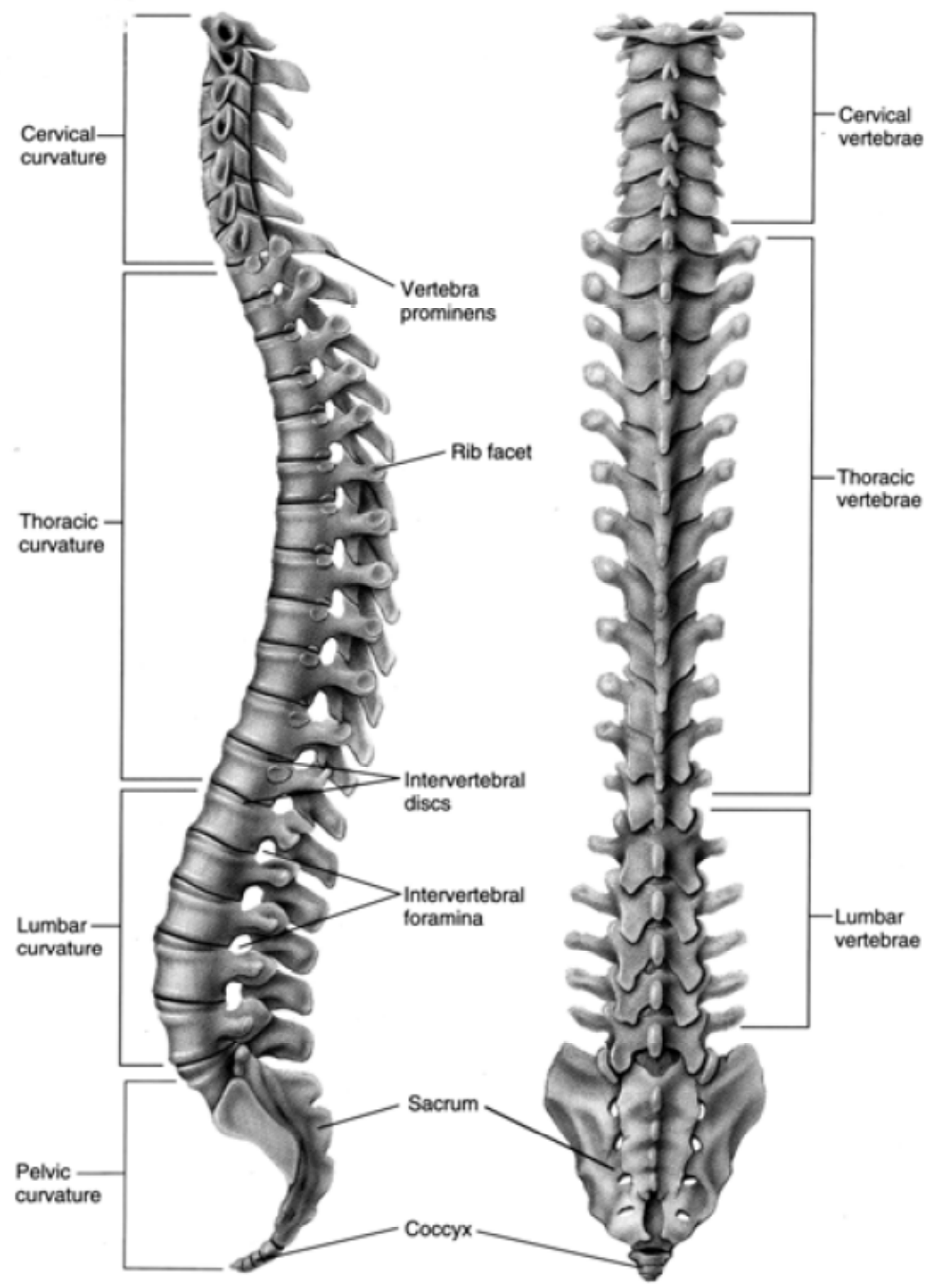

Figure 2.1 The Human Spine. Lateral and posterior views. Reprinted with permission from Butler J, Lewis R, Shier D, eds., 2002, Hole's Human Anatomy \& Physiology, 9th ed., McGraw Hill, Boston. ${ }^{5}$ 


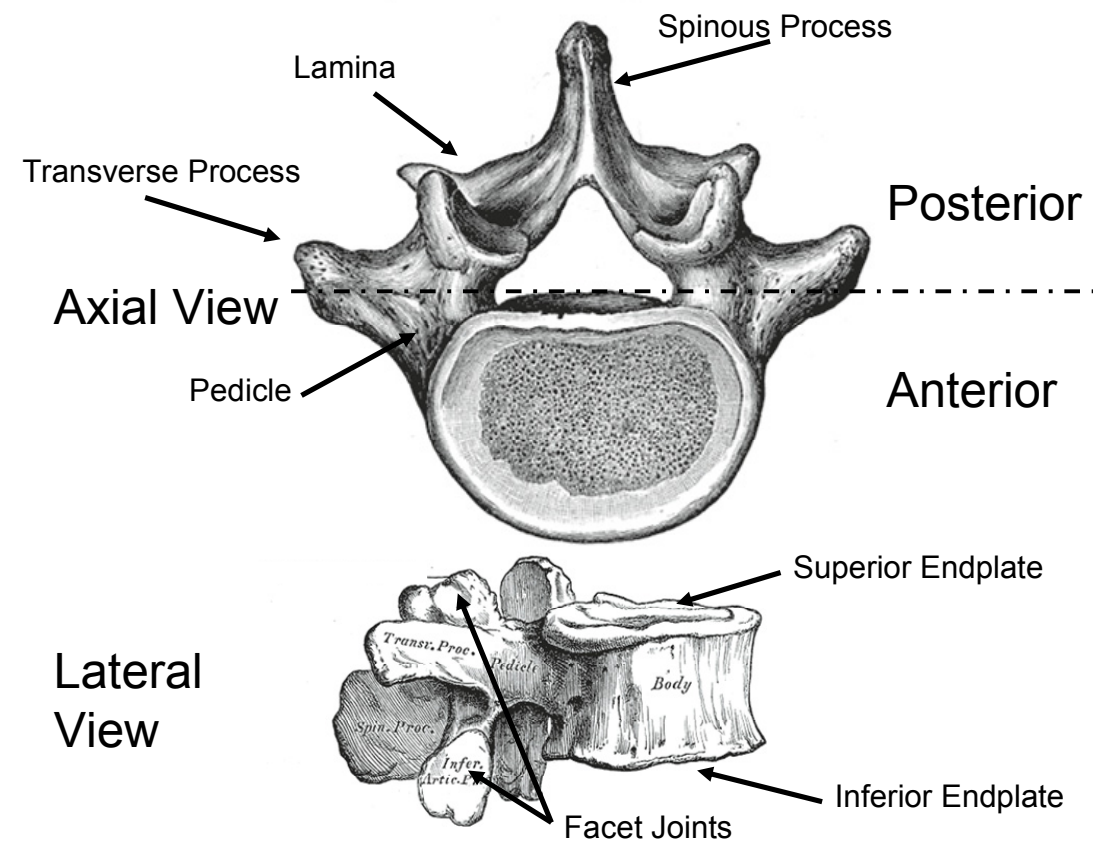

Figure 2.2 Lumbar Vertebral Body. Adapted with permission from Gray, H. Anatomy of the Human Body. Philadelphia: Lea \& Febiger, 1918; Bartleby.com, 2000. www.bartleby.com/107/. Accessed on August 27, 2008. ${ }^{27}$ 
The posterior section of the vertebral bodies includes the pedicle and lamina which, together with the posterior portion of the vertebral body, form the foramen. This space houses and protects the spinal cord as it descends down the spinal column. The transverse processes protrude from the vertebral body laterally and provide insertion points for muscles and ligaments. The structure furthest posterior is called the spinous process. It provides insertion points for several ligaments as well as muscles.

The articulating processes of the spine which sit between the pedicle and lamina are called zygapophyseal joints, but are better known as facet joints. Facets are synovial joints lined with articular cartilage and menisci and surrounded by capsular ligaments. ${ }^{62}$ These joints are susceptible to changes due to aging and injury. The capsules are also heavily innervated with pain receptors and proprioceptive receptors and are often a main contributor to lower back pain in the elderly population. ${ }^{38}$

\subsubsection{Intervertebral Disc}

The disc is comprised of three integrated regions. These include the nucleus pulposus in the center of the disc, the annulus fibrosis which encapsulates the nucleus, and the cartilaginous endplates. The endplate is recognized as part of the disc because collagen fibers are continuous between the annulus and this region. ${ }^{53}$ Fibrocartilage of all layers of the annulus, except the outermost, connects to the vertebral bodies by way of these cartilaginous endplates. The outermost layer of annular fibers connects directly to the vertebral bodies and is also called Sharpey fibers. ${ }^{62}$ Figure 2.3 shows the annulus and nucleus regions of the disc. This figure shows a distinct boundary between the annulus and nucleus, but anatomically there is no such defined boundary. ${ }^{30}$

The annulus is made up of layers of type I and type II collagen fibers. ${ }^{62}$ As many as twenty-five of these layers exist in different regions of the spine. They increase in width and decrease in collagen content moving from the peripheral annulus towards the nucleus. ${ }^{53 ; 54}$ The fibers in these layers lie at alternating angles between layers so that the fibers in one layer are nearly perpendicular to those in its adjacent medial and peripheral layers. The angle that they make with the horizontal plane is approximately 30 to 40 degrees. This lattice provides increased tensile properties and helps prevent loss of attachment during daily activities. ${ }^{54}$ The annulus not only functions to provide attachment for the disc to the endplates and vertebral bodies, but also encapsulates the nucleus material and provides motion in multiple directions.

The nucleus pulposus is a pressurized gelatinous region made up of proteoglycan (glycosaminoglycans), loose type II collagen fibrils, mineral salts, water, and cellular elements. ${ }^{39}$ The pressure in the disc is due to the presence of proteoglycan and water in the nucleus. The proteoglycans are hydrophilic in nature and pull water into the nucleus region by osmosis. The disc material then forms hydrogen bonds with the water molecules in order to retain them in the disc space. This increased water content generates a hydrostatic or intradiscal pressure. ${ }^{39 ; 62}$ Throughout the day, and during vigorous activities, the water content in the disc diminishes, resulting in a loss of 


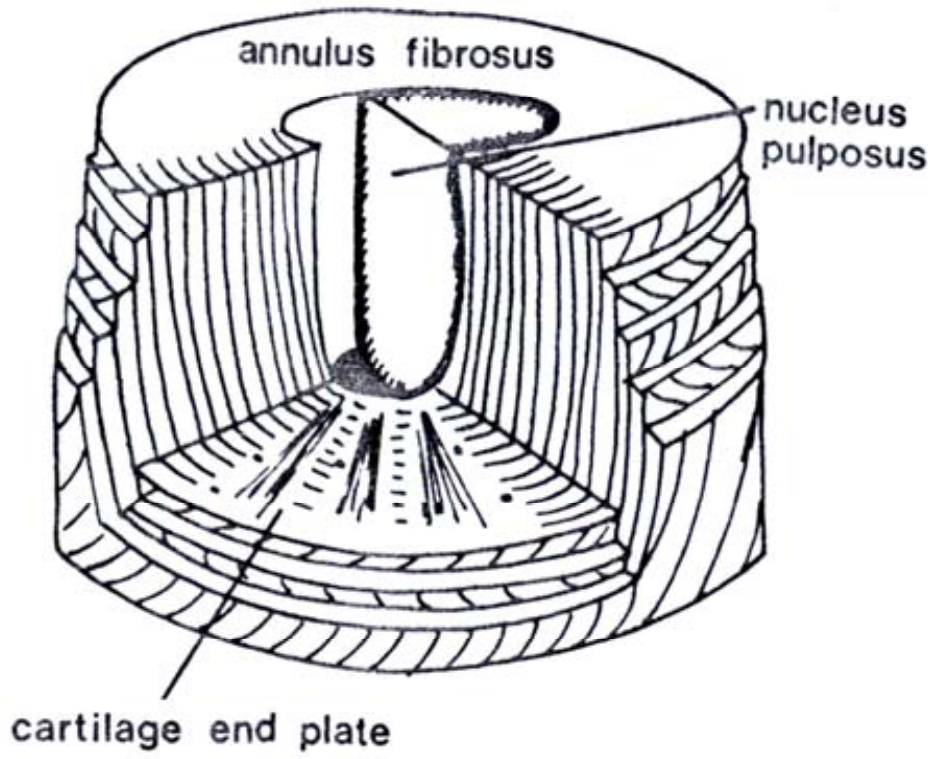

Figure 2.3 Components of the Intervertebral Disc. Reprinted with permission from Humzah MD, Soames RW, Human intervertebral disc: structure and function, The Anatomical Record, 1988; 220: 337-356. ${ }^{30}$ 
intradiscal pressure and disc height. As the disc ages, proteoglycan content in the nucleus decreases, resulting in loss of disc height and improper joint motion and function. ${ }^{60}$

\subsection{Spine Mechanics}

\subsubsection{Intervertebral Disc Mechanics}

The hydrostatic pressure of the annulus-nucleus construct is important in balancing and distributing external forces over the surface of the vertebral body endplate and throughout the length of the spine. One of the main functions of the nucleus pulposus is to transform compressive axial forces into distributed radial forces acting on the annulus, ${ }^{62}$ as shown in Figure 2.4. This transformation of axial forces into an intradiscal pressure allows the disc to function as a shock absorber and preserve normal joint motion.

The disc also plays a critical role in balancing forces caused by the natural bending of the spinal column. Figure 2.5 demonstrates this concept. As the spine is bent forward in flexion the posterior portion of the disc is put into tension and the anterior portion of the disc is put into compression. The two loads counteract each other to maintain spinal stability.

\subsubsection{Basic Spine Mechanics}

The weight of the upper torso, muscle activity, and external loads are the main contributors to the loading associated with the in-vivo lumbar spine. A simplified case of physiologic loading of the lumbar spine is shown in Figure 2.6. In the figure, $F_{B W}$ is the force vector which acts through the center of mass of the torso. $F_{B W}$ induces a different bending moment at each individual level of the spine, where the bending moment about a point is the product of the force times the perpendicular distance to the point of rotation. Due to the natural lordosis of the lumbar spine, the offset distance " $\mathrm{d}$ " from the line of action of force $\mathrm{F}_{\mathrm{BW}}$ to the center of the disc is different for each vertebral level, resulting in a different bending moment at each individual level of the spine. The line of gravity of the upper torso is thought to lie along a line drawn from the auricle of the ear to the center of the femoral head. ${ }^{5}$ The vector shown in Figure 2.6A passes slightly anterior to this generally accepted location in order to effectively illustrate the point that the bending moment is different at each individual level.

$\mathrm{F}_{\mathrm{BW}}$ has two component forces: $\mathrm{F}_{\mathrm{A}}$ is the axial load that acts normal to the plane of the disc, and $\mathrm{F}_{\mathrm{S}}$ is the shear force that acts along the plane of the disc. The angle that the superior end plate of the vertebral body makes with the horizontal axis is described as theta and corresponds to the angle $\theta$ shown in Figure 2.6B between the vertical $F_{B W}$ force and the axial force $\mathrm{F}_{\mathrm{A}}$. The component forces can be calculated as: 


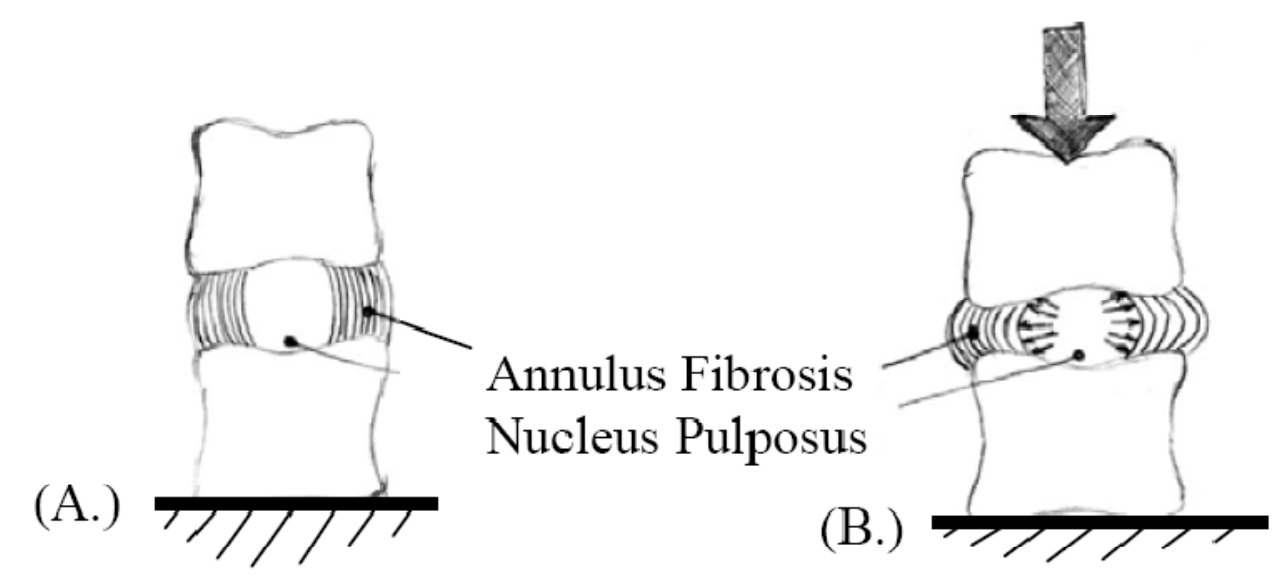

Figure 2.4 Disc Pressure. Pressure distribution in the disc (A.) under zero load and (B.) radially distributed when exposed to a compressive load. Reprinted with permission from Bonin H, In-vitro analysis of the instantaneous center of rotation in a human cervical spine model using a spine robot, Thesis, 2006, University of Tennessee Health Science Center. ${ }^{4}$ 

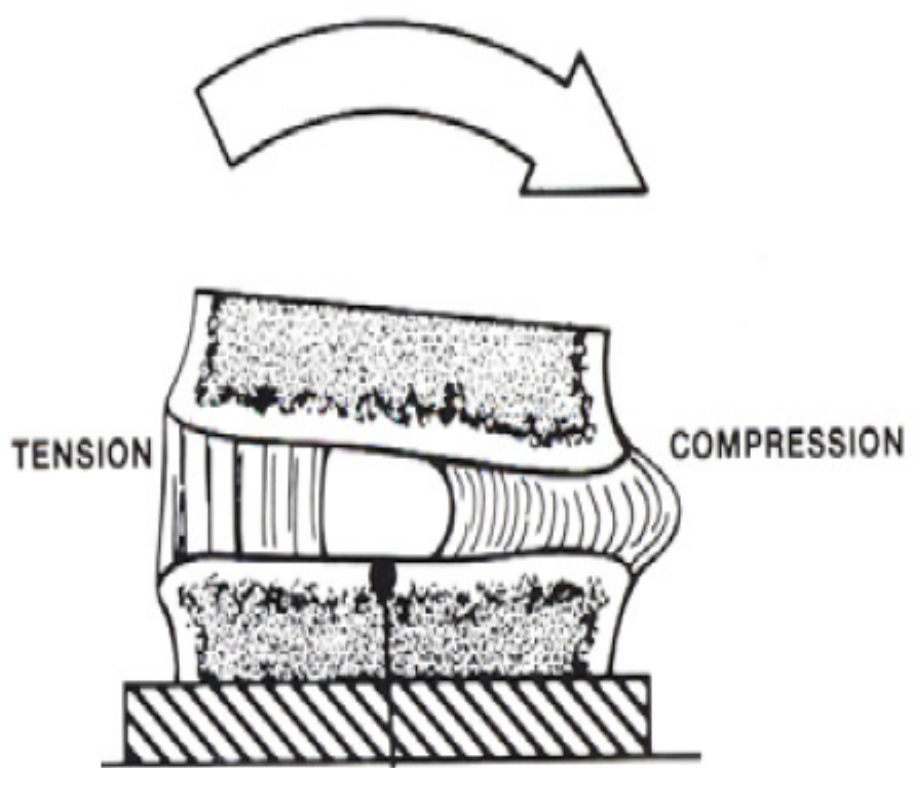

Figure 2.5 Balance of Tension and Compression in the Intervertebral Disc. Adapted with permission from White AA, Panjabi MM. Clinical Biomechanics of the Spine. 2nd ed. Philadelphia: J.B. Lippincott Co., 1990:34-64, (C) Lippincott Williams \& Wilkins. ${ }^{62}$ 


\section{Body Weight Force Vector $\left(\mathrm{F}_{\mathrm{BW}}\right)$}

\section{(A.)}
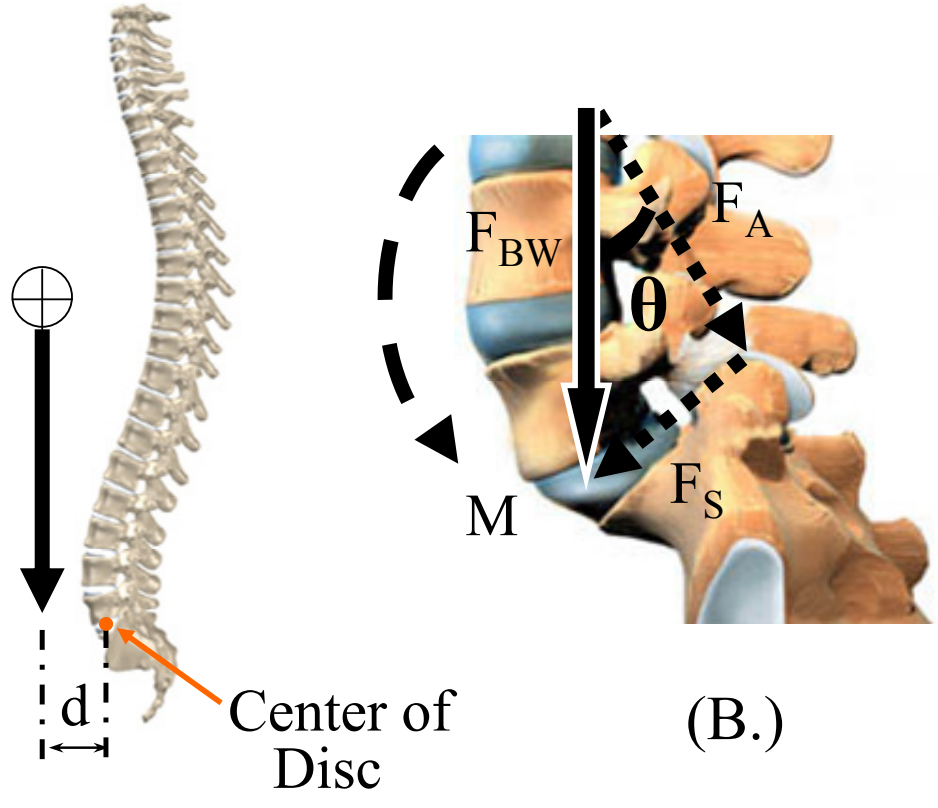

(B.)

Figure 2.6 Spinal Loading Mechanics. The action of the body weight force vector $\left(\mathrm{F}_{\mathrm{BW}}\right)$ at the L5-S1 MSU induces a bending moment $\left(\mathrm{M}=\mathrm{F}_{\mathrm{BW}} * \mathrm{~d}\right)$ and compressive force $\left(\mathrm{F}_{\mathrm{BW}}\right)$. The compressive force vector $\left(\mathrm{F}_{\mathrm{BW}}\right)$ has two components: Shear force $\left(\mathrm{F}_{\mathrm{S}}\right)$ along the disc plane and Axial Force $\left(\mathrm{F}_{\mathrm{A}}\right)$ normal to the disc plane. As the spine flexes or extends, the moment arm varies and the magnitude of the MSU forces change accordingly. 


$$
\begin{aligned}
& F_{A}=F_{B W} \times \cos \theta \\
& F_{A}=F_{B W} \times \sin \theta
\end{aligned}
$$

As the angle $\theta$ increases, more of the body weight is transferred to the spine as shear force. The intact human MSU resists the physiologic loads described above and maintains stability in the passive spinal column by recruiting the involvement of the disc, ligaments, and facet joints. As discussed in Section 2.2.1 the disc absorbs and distributes most of the axial forces applied to the spine. The shear force is thought to be resisted principally by the facet joint complex.;62 Due to recent failures of next generation spinal devices, however, there is a renewed interest in the biomechanics community of how the spine compensates for the shear force and what effects disruptions to the spinal column have on the shear force. ${ }^{32 ; 40}$ The bending moment is resisted by the disc, ligaments, and facet joint. Dickey and Gillespie showed, using a porcine lumbar spine, that the facet complexes provided $72 \%$ of total resistance to the peak bending moment in extension and only the last $20 \%$ of resistance during flexion motion. ${ }^{20}$ This indicates that in flexion the posterior ligaments and disc are heavily recruited to resist the bending moment. Whenever the intact spinal construct is disrupted by disease, trauma, degeneration, or surgical intervention, the ability of the MSU to resist physiologic loads and maintain spinal stability is affected. ${ }^{7}$

The basic mechanics described in this section are also affected by the location of the center of mass of the upper torso and by the anatomical structure of the spine; both of which can vary greatly between individuals and can be altered due to disease or trauma. This loading scheme is further complicated when the muscle activity, out-of-plane loads and moments, and external loading is taken into account, but it is obvious that even in the simplest case the shear force, axial force, and bending moment on the spine can be significant.

\subsection{Spine Kinematics}

In MSU spine kinematics the vertebral bodies are considered to be rigid bodies. Figure 2.7 shows the six degrees of freedom required to fully describe kinematic motion of the lumbar spine. The superior vertebral body can rotate about and translate along the three orthogonal axes with respect to the inferior vertebral body. Kinematics parameters such as the neutral zone and range of motion are often used in in-vitro biomechanical testing protocols to evaluate the effects of spine devices and instrumentation.

\subsubsection{Neutral Zone}

The neutral zone was originally described by Panjabi ${ }^{45}$ as the region of intervertebral motion around the neutral posture where little resistance is offered by the passive spinal column. In Figure 2.8 the neutral zone is the linear region where an increase in deformation results in little change in the load. Once the deformation crosses 


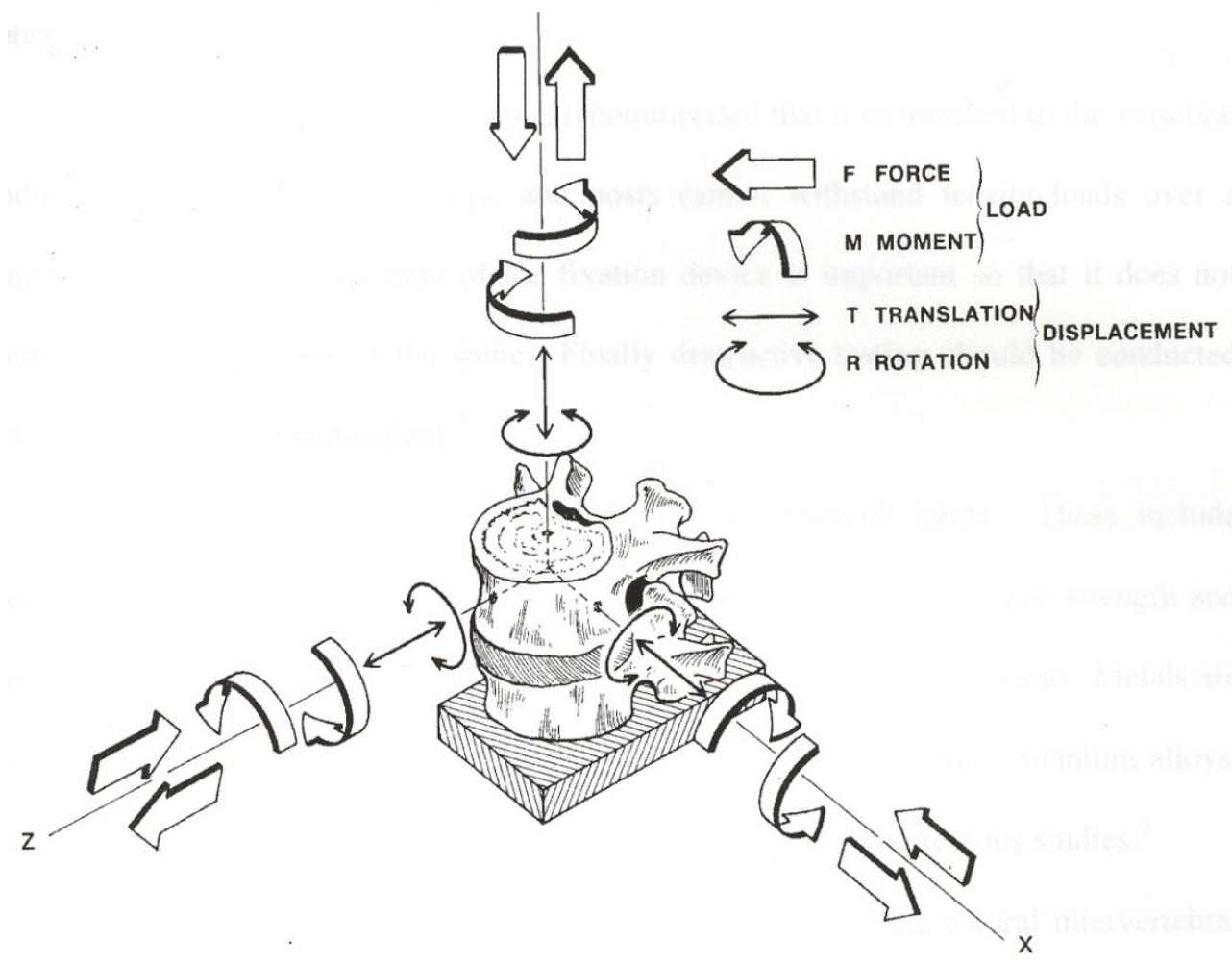

Figure 2.7 Degrees of Freedom of Spinal Movement. Schematic of the individual degrees of freedom necessary to fully describe individual vertebral motion. Reprinted with permission from White AA, Panjabi MM. Clinical Biomechanics of the Spine. 2nd ed. Philadelphia: J.B. Lippincott Co., 1990:34, C Lippincott Williams \& Wilkins. ${ }^{62}$ 


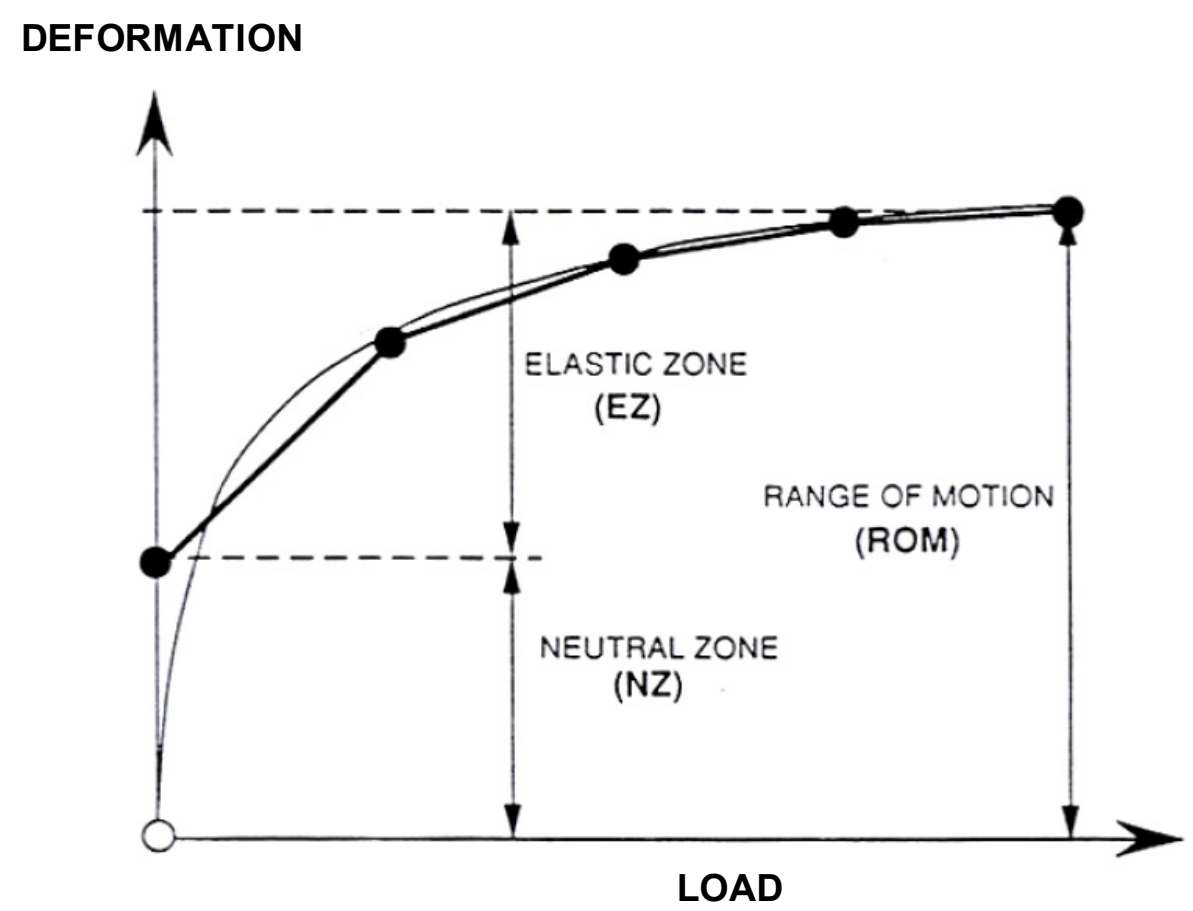

Figure 2.8 Neutral Zone and Elastic Zone. Neutral zone and elastic zone depicted on a flexibility plot (Deformation vs. Load). Reprinted with permission from Panjabi MM, The Stabilizing System of the Spine: Part II. Neutral Zone and Instability Hypothesis, Journal of Spinal Disorders, 1992; 5(4): 390-397. ${ }^{45}$ 
increased or decreased neutral zone and what the effects of such changes have on the stability of the spine. Additionally, since the neutral zone measurement involves only very low load ranges it can at best define stability in that range and cannot ultimately be used to fully characterize the effects of spine devices and instrumentation on the spine.

\subsubsection{Range of Motion}

Range of motion (ROM) is the most common kinematic parameter used in biomechanical testing protocols to evaluate spinal devices in-vitro. ${ }^{9 ; 14-16 ; 22 ; 35 ; 43}$ ROM refers to the measured rotational displacement resulting from a specified load input. In figure 2.8 the ROM is defined as the sum of the motion in the neutral and elastic zones. ROM can be reported as the total motion of the spinal segment or, more commonly, as the single segment contribution to the total motion. ROM comparisons between harvested specimens and instrumented, or surgically altered, specimens provide a quantitative analysis of the effects of spinal devices and instrumentation on spinal motion.

As an example, comparisons of pre- and post-operative ROM of a total disc replacement (TDR) device as a clinical indication of surgical outcome has been proposed but current studies present conflicting evidence. ${ }^{6 ; 61 ; 64 ; 66}$ Due to the apparent contradictions in the literature, ROM as an indication of clinical outcome has not been widely accepted. Though ROM is accepted and effective for comparative biomechanical studies of spine motion, it is not sufficient to characterize the total effect of a spinal implant or instrumentation on the spine.

\subsection{Center of Rotation}

The center of rotation (COR) of a spinal segment is the point in space that maintains a constant distance from every point on the superior vertebral body throughout the entire motion. It is also the point in the system that has zero velocity. ${ }^{29}$ The instantaneous center of rotation (ICoR) is the COR for each instance of time over a prescribed motion, and changes as a function of time and displacement. The ICoR is also called the instantaneous axis of rotation (IAR), which refers to the axis which passes through the ICoR and is perpendicular to the plane of motion, in this case the sagittal plane.

\subsubsection{Calculating the Center of Rotation}

Motion about a COR in a plane can be fully described as two translations and a rotation. During biomechanical testing the ROM of a spinal segment is often constrained to a single plane of motion to simplify testing. As such, the location of the COR can be calculated from knowledge of the translation and rotation of a rigid body in the system. Figure 2.9 shows the most common way to calculate the two-dimensional COR. Two 


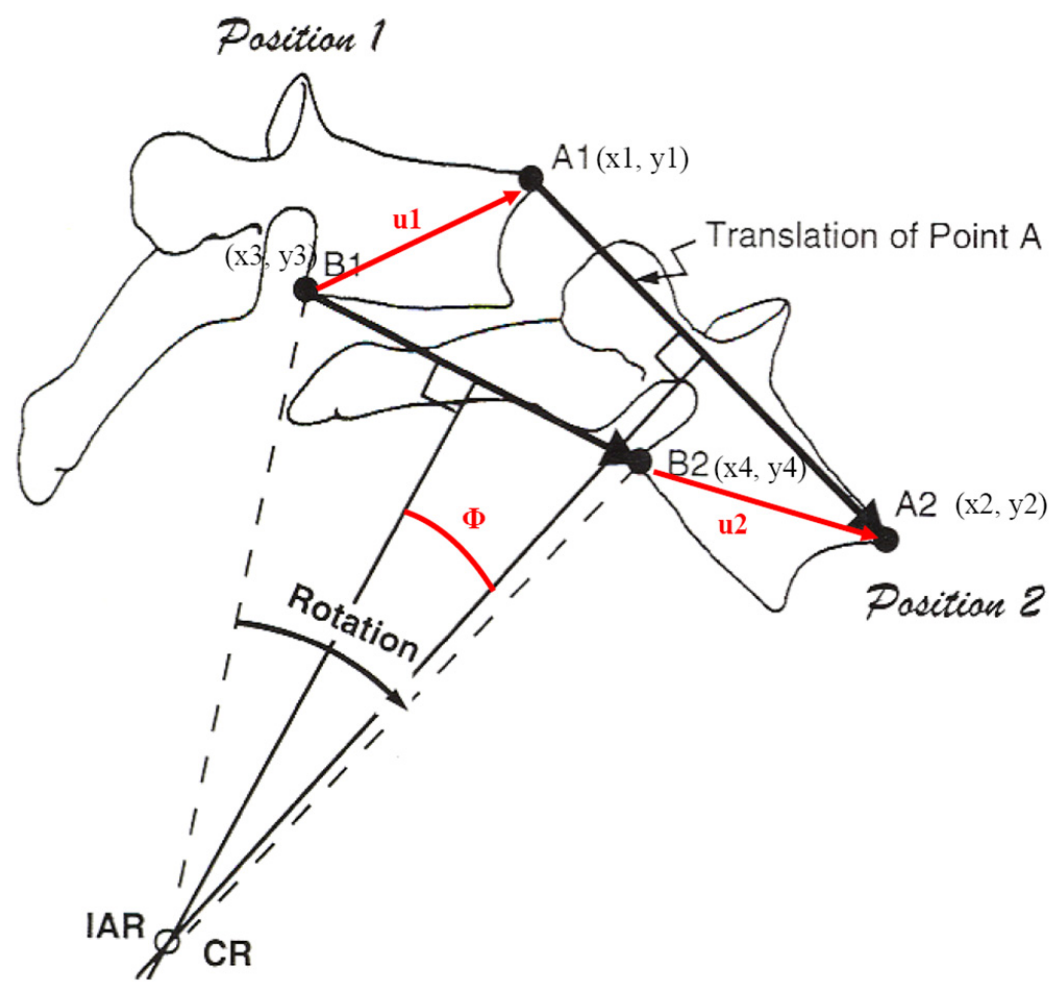

Figure 2.9 Calculation of the COR. Method of calculating the Instantaneous Axis of Rotation (IAR) or Center of Rotation (ICoR) using perpendicular bisectors of common vertebral body points at multiple instances in time. Adapted with permission from Clark CR, ed., 1998, The Cervical Spine, 3rd ed, Lippincott-Raven Pub, Philadelphia. 
bony landmarks (A1 and B1) are identified and tracked throughout motion of the upper vertebral body. A position vector is initially drawn connecting the two landmarks. This vector is shown as $\mathbf{u} 1$ in the figure. At the next analysis time interval two vectors are drawn to connect the initial landmark locations (A1 and B1) and the current landmark locations (A2 and B2). A perpendicular line is drawn to each of these vectors. The point of intersection of the two perpendiculars is the COR for that time interval, or the ICoR. Crisco et al. ${ }^{11}$ presented the equations used to calculate the location of the COR from this model. Coordinates are assigned to the two positions of the bony landmarks: A1(x1, y1), $\mathrm{A} 2(\mathrm{x} 2, \mathrm{y} 2), \mathrm{B} 1(\mathrm{x} 3, \mathrm{y} 3)$, and $\mathrm{B} 2(\mathrm{x} 4, \mathrm{y} 4)$. Position vectors $\mathbf{u} 1$ and $\mathbf{u} 2$ can be calculated as:

$$
\begin{aligned}
& u 1=\left(A 1_{x}-B 1_{x}\right) i+\left(A 1_{y}-B 1_{y}\right) j \\
& u 2=\left(A 2_{x}-B 2_{x}\right) i+\left(A 2_{y}-B 2_{y}\right) j
\end{aligned}
$$

Equation 2.3

Equation 2.4

and the angle between these position vectors is equal to the angle of rotation whose cosine is given by:

$$
\cos \phi=\frac{u 1 \times u 2}{|u 1| \times|u 2|}
$$

Equation 2.5

Phi $(\Phi)$ is the pure rotation angle of the superior vertebral body with respect to the inferior vertebral body. If $\Phi$ and the initial and final positions of either of the bony landmarks are known then the location of the COR can be calculated. In terms of A1 and $\mathrm{A} 2$, the COR location is calculated as:

$$
\begin{aligned}
& X_{C O R}=\frac{1}{2}(x 1+x 2)+\frac{(y 1-y 2) \sin \phi}{2(1-\cos \phi)} \\
& Y_{C O R}=\frac{1}{2}(y 1+y 2)-\frac{(x 1-x 2) \sin \phi}{2(1-\cos \phi)}
\end{aligned}
$$

Equation 2.7

\subsubsection{Location of the Center of Rotation of the Spine}

Equations 2.3-2.7 described in this section have been used in in-vivo and in-vitro testing protocols to calculate the ICoR. In-vitro analysis of the ICoR is generally done by attaching optical diodes to the superior vertebral body and tracking spine motion using a camera system. ${ }^{19 ; 58}$ Data from the camera system and the equations presented above are used to calculate the ICoR for the full range of motion. In-vivo measurements of the ICoR are generally made by taking a series of radiographic images of a flexion or extension motion and then overlaying the radiograph with perpendicular bisectors to common bony landmark locations. To reduce the exposure of the patient to radiation, it is a common practice to take images at extreme flexion and extension ranges and then calculate an average COR for the entire motion. ${ }^{51 ; 52 ; 56 ; 65}$ Pearcy and Bogduk ${ }^{51}$ reasoned that since the COR of a full flexion-extension motion correlated with the separate CORs of neutral to flexion and neutral to extension motions that the average COR value was sufficient to describe lumbar kinematics. However, researchers conducting other in-vivo 
and in-vitro studies claim that the ICoR is transient throughout the full range of motion of the lumbar spine. ${ }^{41 ; 5}$ Bonin showed that, in the cervical spine, the ICoR is not stationary and does in fact move throughout flexion and extension motion. ${ }^{4}$ Furthermore, Grassman et al. demonstrated in-vitro that selection of a COR for axial rotation that does not coincide with the physiologic COR can adversely affect the loading in the spine. Selection of a COR that is non-physiologic leads to a decreased ROM and increased spinal loading. ${ }^{26}$ Dooris et al. used a finite element model to show a similar result with respect to flexion-extension rotation. ${ }^{21}$

The fact that so many different methods of measuring the ICoR exist shows that there are many challenges associated with measuring a true physiologic ICoR in the spine. In-vivo studies are limited by patient exposure to radiation and errors in physically selecting bony landmarks in multiple radiographs of varying quality. In-vitro studies are limited by errors in the measurement techniques. ${ }^{13}$ Crisco et al. hypothesized that errors associated with measuring and calculating the ICoR have led to the current contradictions of data in the literature. ${ }^{11}$

Different studies in the literature have put the average COR location at varying locations throughout the spinal segment. Many researchers agree that in the lower lumbar segments (L3-S1) the COR is generally located in the posterior third of the vertebral body, ${ }^{51 ; 52 ; 56 ; 57}$ though a study of L5-S1 segments by Rousseau et al. showed CORs more centrally located in flexion rotation. ${ }^{55}$ Figure 2.10 summarizes the approximate locations of the COR in the lumbar MSU by several researchers. The elliptical zones represent either the centrodes surrounding the location of multiple instantaneous centers of rotation throughout a range of motion or the error zone involved with the calculation of the COR.

The location of the COR has been correlated with degenerative disc disease, spondylolisthesis, and trauma. ${ }^{56 ; 57}$ A COR location that deviates from the normal location is thought to indicate abnormal spine motion due to spine pathologies, but as per the discussion above, the error involved with measuring the COR has prohibited clinicians from accepting abnormal COR location as an indication of pathology.

\subsection{Conventional Spine Biomechanical Testing Protocols}

Biomechanical testing of the spine originated as an extension of biomechanical testing of other joints. Methods used to study synovial joints were expanded to test complex spinal segments. Panjabi et al. originally categorized spinal biomechanical testing methods as either flexibility (load-controlled) or stiffness (displacement controlled). ${ }^{44}$ To this day there exists a controversy within the spine biomechanics community as to which testing protocol offers the best method of testing. Researchers backing the load control protocol are quick to point out that the flexibility protocol 


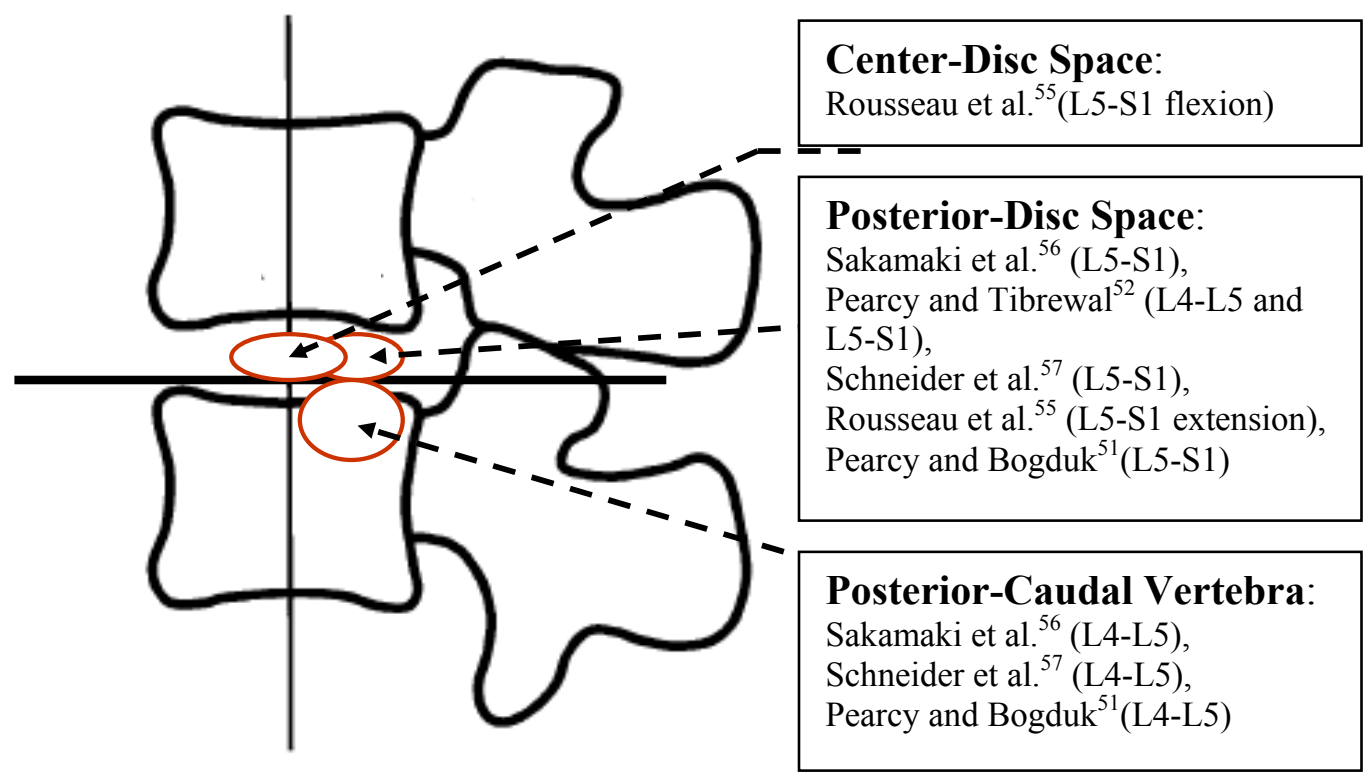

Figure 2.10 Approximate Locations of the Center of Rotation. Locations shown are for the lower lumbar segments (L4-L5 and L5-S1). 
provides a standard approach and offers increased control over the complex variables involved with spine testing. ${ }^{24}$ They reason that the flexibility protocol should be used to first compare devices to those which are already approved by the Food and Drug Administration (FDA) and then stiffness protocols could be used to further investigate the devices. ${ }^{24}$ Proponents of the stiffness protocol argue that the flexibility protocol does not replicate in-vivo conditions and that it is possible that misleading results can be obtained from that method. Their main point is that a displacement control method allows researchers to better mimic physiologic motions or loading conditions and to report data that more accurately represent in-vivo conditions. ${ }^{24}$ The next two sections explain more about each of these protocols and some of their strengths and weaknesses.

\subsubsection{Flexibility Protocol}

The flexibility protocol applies a pure bending moment input to a spinal segment and measures the motion response in terms of ROM and neutral zone. A typical test setup to induce a pure moment input is shown in Figure 2.11. This particular system uses a pulley attached to the superior vertebral body and a cable system to induce a pure moment input. As the pulley rotates, the unconstrained spine is free to move and the pulley-cable system adjusts to assure that the cables remain parallel and that the input is a pure moment with no off-axis loading. Other researchers have implemented the flexibility protocol using robotic simulators and commercial testing systems. The main concern of researchers using the flexibility protocol is to be sure to maintain a pure moment input; otherwise the basic underlying assumptions of the protocol are not valid.

The flexibility protocol can be used to study planar motion or, in the case of multi-directional studies, it can be utilized by sequentially applying a pure moment in each of the three rotational degrees of freedom described in Section 2.3. Traditionally, multi-directional studies were conducted using fixtures which could be adjusted to allow bending in each of the three major anatomical planes (flexion-extension, lateral bending, and axial rotation), but more recently several researchers have developed multi-axis testing machines which can provide a pure bending moment input in any of the planes without removing the specimen. ${ }^{12 ; 49 ; 63}$ It is important to note that even though these test machines have been marketed as "Six Degrees of Freedom" testing systems, almost universally, only one degree of freedom is actively controlled at a time and the testing systems have been used to run the flexibility protocol.

In an effort to use the pure moment input described above to study more advanced kinematics and mechanics properties of non-fusion devices, researchers have sought to modify the traditional flexibility protocol using different fixtures, control schemes, test designs, and actuator setups. ${ }^{12 ; 33 ; 37}$ To study the adjacent level effects (ALE), Panjabi modified the original flexibility protocol and renamed it the "hybrid protocol. ${ }^{43}$ " ALE refers to the hypothesis that increased degeneration of a spinal MSU, adjacent to an instrumented MSU, occurs due to the transfer of load and ROM from a fused segment to the adjacent level. Since its inception, the hybrid protocol has been widely used to study spinal kinematics and ALE, but there remains a controversy among different researchers 


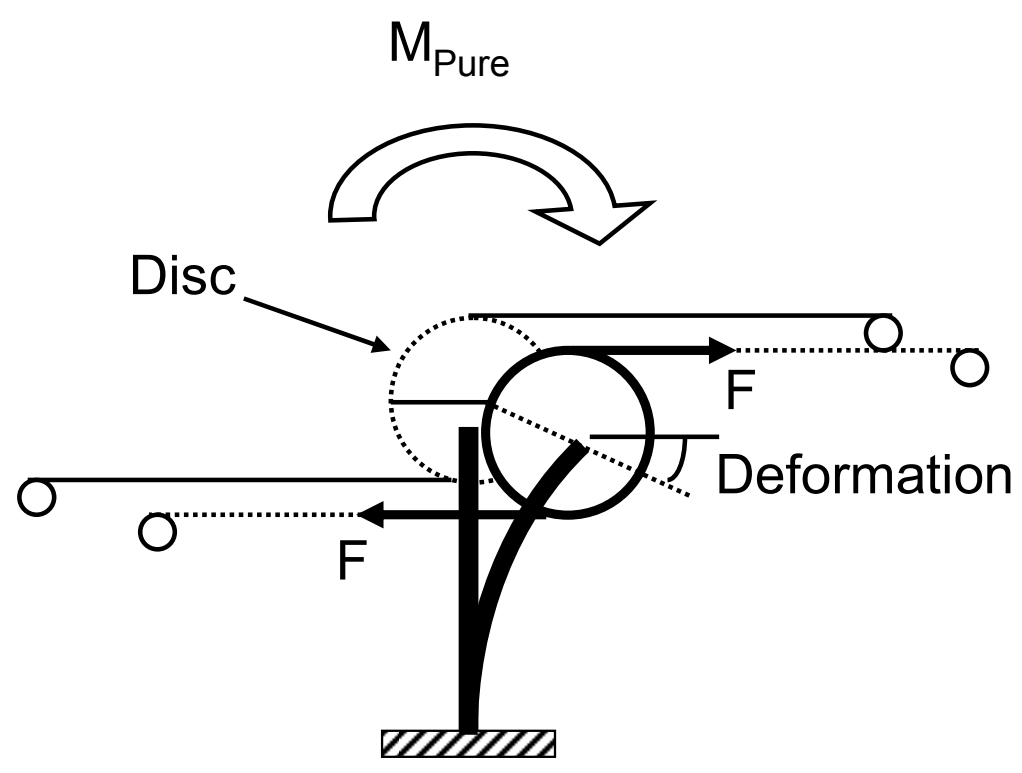

Figure 2.11 Setup to Induce Pure Moment Input. Adapted with permission from Panjabi M, Hybrid Multidirectional Test Method to Evaluate Spinal Adjacent-Level Effects, Clinical Biomechanics, 2007, 22:257-265. ${ }^{43}$ 
as to the quality of the data produced. In a letter to the editor of Clinical Biomechanics, Dr. Neil Crawford challenges the underlying assumption made by Panjabi in the hybrid protocol. Namely, the claim made by Panjabi that, "after surgery to fuse the spine or replace a disc, the patient would move his/her spine to the same limits of motion as though the spine were healthy and normal." Crawford goes on to say that there is clinical evidence supporting the fact that the patient does not move to the same global ROM after surgery as they did before surgery. ${ }^{10}$ Thus, the underlying assumptions of the hybrid protocol have not been validated and are still controversial to some researchers.

The benefit of the flexibility protocol is its ease of use and the ability to perform standardized comparative testing; however, some limitations exist with this approach. Although the flexibility and hybrid methods provide a standard approach for comparing different lumbar spinal devices, and may be acceptable for testing fusion instrumentation, the testing approach is not well suited for studying modern spinal devices and surgical techniques. This is especially evident when considering the effects of a device on the shear and compressive forces at each level of the segment, the motion contribution of a non-fusion device, and/or the effects of a non-fusion device on the adjacent segments. ${ }^{43}$

Another limitation is introduced when the flexibility and hybrid protocols are coupled to a follower load. ${ }^{50}$ Use of the follower load can result in artificial stabilization of the spinal level, which could mask the effects of the device on the segment. ${ }^{18}$

\subsubsection{Stiffness Protocol}

The stiffness protocol has also been used to study fusion and non-fusion spinal devices. ${ }^{16 ; 31 ; 34}$ As mentioned above, the stiffness protocol has a known kinematic input and measures load and moment response of the spinal segment to that motion. The kinematic input can be a displacement or rotational input. Many researchers have implemented this protocol because it can be run with a commercial testing system with a single DOF.

DiAngelo et al. have previously developed a displacement-based "Eccentric Load protocol", using a single vertical actuator against a horizontally mounted lever arm at the top of the test specimen to create sagittal plane bending and motion. ${ }^{15}$ Figure 2.12 illustrates this test setup. The actuator applies a compressive load eccentric to the long axis of the spine causing the spine to flex or extend under a combined compressive load and bending moment. The actuator is allowed to translate unconstrained along the horizontal lever arm by using a slider bearing connection so that the force input is always normal to the top of the specimen and no shear force is applied. Using this testing method, a more physiologic response in the rotational involvement of each MSU occurs throughout the lumbar spine. However, even though the eccentric loading method induces a physiologic rotational response across the intact lumbar spine, this method may not have the sensitivity to study the intervertebral loading mechanics and kinematic requirements of compliant implants or surgically altered disc conditions. 


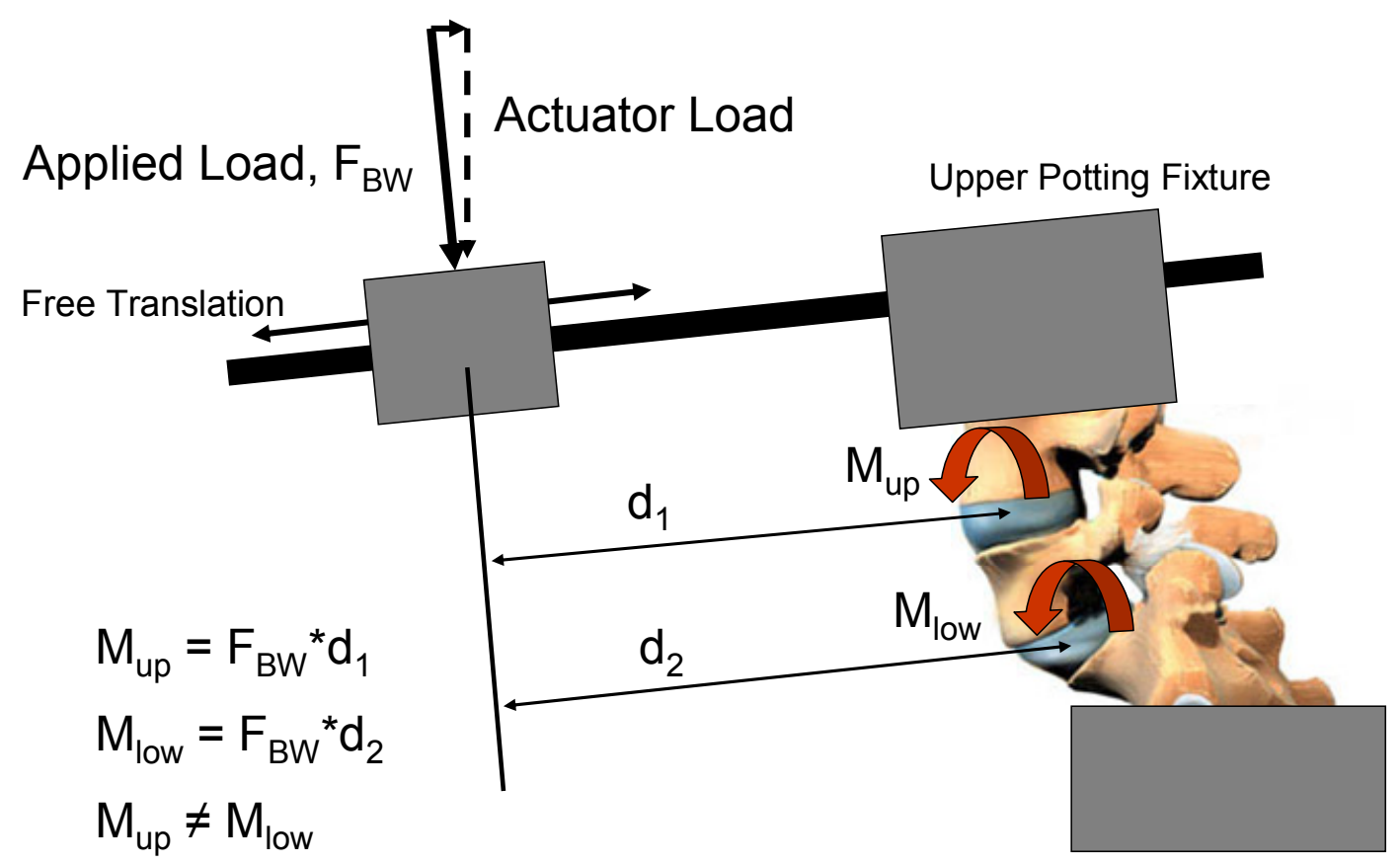

Figure 2.12 Setup of Eccentric Load Protocol. A vertical actuator is used to apply a load to a horizontal lever arm attached to the upper potting fixture. The actuator is unconstrained along the lever arm so that the applied load $\mathrm{F}_{\mathrm{BW}}$ is always normal to the top of the specimen and there is no applied shear force. 
Another version of the stiffness protocol described by Panjabi is the application of a constrained rotation input to the specimen. ${ }^{43}$ Panjabi points out that in order to apply a rotation motion to the specimen, the COR would have to be defined for the protocol. As discussed in Section 2.4, defining a COR that is physiologically correct for each specimen would require a biomechanical test to find the COR. Additionally, selection of a COR that does not coincide with the physiologic COR may over-constrain the segment and result in a non-physiologic load response. Furthermore, as discussed in Section 2.4, the ICoR is not a stationary point throughout the flexion-extension ROM, and it would be altered from its original location once the spine had been instrumented with a spinal device. These limitations have restricted the use of rotational input testing protocols in the past. 


\section{Chapter 3: Materials and Methods}

The materials and methods chapter is separated into four sections. Section one covers the steps to prepare human cadaveric tissue for in-vitro biomechanical testing. The second section gives basic information on the testing system used and a brief explanation of The University of Tennessee (UT) Spine Robot. The third section gives a detailed explanation of the kinematics-based testing protocol. Section four covers how the data was managed and analyzed.

\subsection{Tissue Preparation}

Six fresh human cadaveric lumbar spines were procured from the Medical Education Research Institute (MERI, Memphis, TN). The average age of the specimens was 30.3 years. Specimens were wrapped and sealed in plastic bags and frozen at -20 degrees $\mathrm{C}$ until further preparation.

A General Electric C-arm (GE Healthcare, Chalfont St. Giles, United Kingdom), located at the Medical Education and Research Institute (Memphis, TN), was used to radiographically evaluate the quality of the lumbar segments. Only specimens void of abnormalities or disc disease were identified for use as test specimens. From this assessment, seven viable MSUs were identified for use. The specimens were thawed and re-radiographed in order to measure the orientation of the neutral specimen. This was done so that once the spine had been dissected and potted the physiologic orientation was preserved. The neutral orientation is described as that posture which minimizes the internal stresses and muscle activity of the intact spinal segment. ${ }^{62}$ The multi-level lumbar spine (L5-S1) was held in a neutral upright alignment with L1 horizontal and each segment's disc orientation measured from the radiographs of the full segment. Figure 3.1 illustrates how these measurements were made. Image J software (NIH, public domain) was used to measure the neutral disc angle before and after the potting procedure.

\subsubsection{Disarticulation of the Cadaveric Tissue}

Each MSU included two vertebral bodies with an intact intervertebral disc and connecting ligaments. During dissection, excess muscle and tissue were removed and care was taken to maintain as much of the relevant soft tissue as possible. ${ }^{17}$ The potting procedure used in the lab has been described in detail in other studies and will not be belabored here but instead only new or important aspects of the procedure will be discussed. ${ }^{4 ; 58}$ One concern during the potting procedure was the ability of the potting material to adhere to the biological tissue. Special care was taken to remove the intervertebral disc and soft tissue from the superior and inferior endplates of the MSU in order to provide more cortical bone exposure and increased fixation strength. 


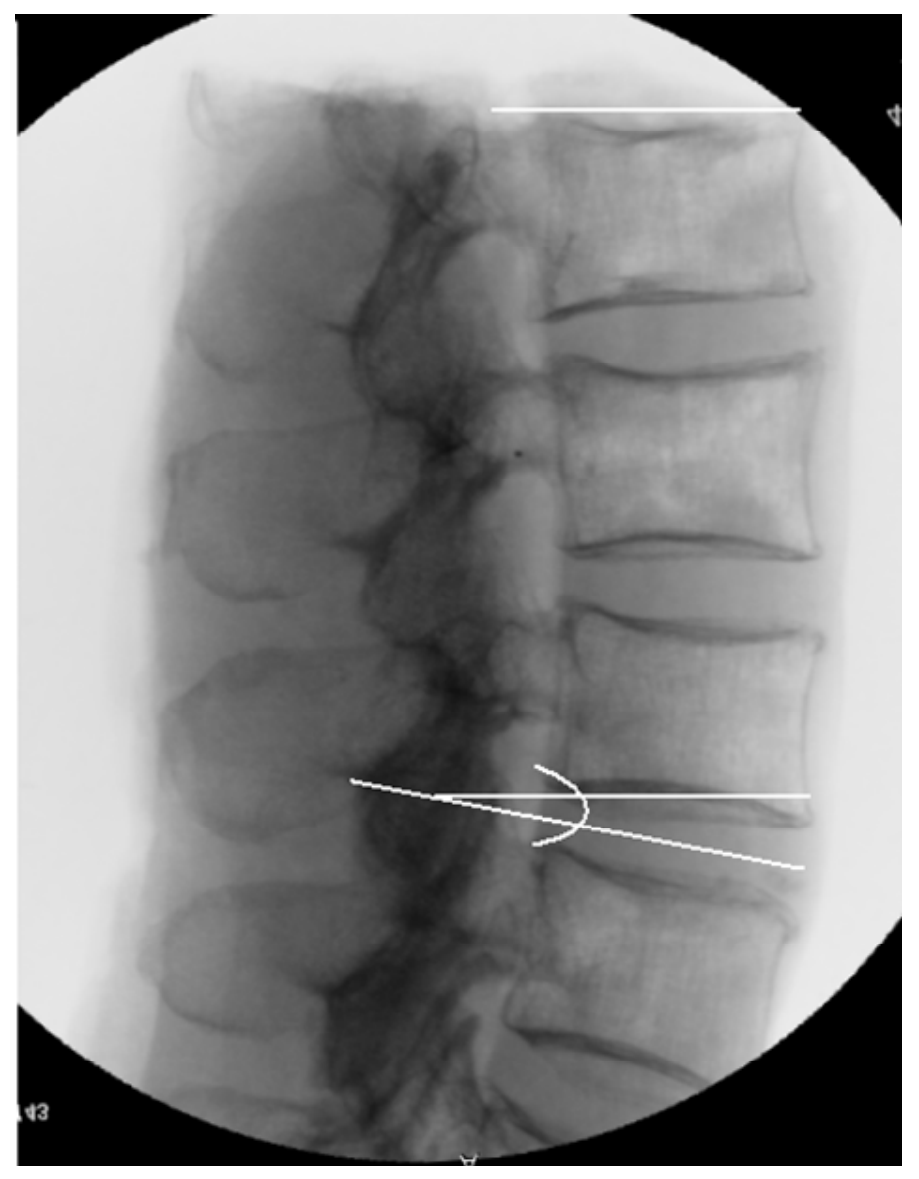

Figure 3.1 Full Specimen Radiograph Used to Measure Alignment of Segment. 


\subsubsection{Specimen Mounting}

To increase the fixation strength of the potting material to the specimen, small wood screws were drilled into hard cortical bone in various locations on the ends of the MSUs. Each specimen was then aligned in a cylindrical pot, with particular attention paid to the neutral alignment, and a low melting point bismuth alloy (Small Parts, Miami Lakes, FL) was poured into the fixture to create a secure mount for the MSU. Cold water was poured in around the mold in order to prevent damage to the tissue from the heat. The specimens were then inverted and hung from a fixture which was designed to ensure that the bottom pot of the specimen would be parallel to the original pot. The procedure was repeated and the specimens were then wrapped and re-frozen at -20 degrees $\mathrm{C}$ until a final round of radiographs could be taken.

\subsubsection{Grid Development}

Since the physiologic COR is not a well-defined parameter, and selection of the COR is essential to providing a physiologic response, ${ }^{43}$ a point grid was created to approximate several commonly accepted COR locations. After thawing the potted specimens, a final round of radiographs were taken in the sagittal plane. When the specimens were inserted into the robot the pots were parallel; since the specimens were aligned during the potting procedure with parallel pots it was important to measure all grid points with the spine in the same orientation. The jig used in the potting procedure was used to secure the specimens during fluoroscopy so as to maintain the alignment of the pots. The images were imported into Image $J$ software so that measurements could be made and a grid created. A caliper set at $10 \mathrm{~mm}$ was radiographed with each of the specimens as in Figure 3.2. This acted as a calibration object for use with the software. For each image, the distance between the working ends of the caliper were measured and image $J$ output a length in terms of pixels. The actual length of $10 \mathrm{~mm}$ was then input as a calibration value which the software used to set a scale for the image in $\mathrm{mm} / \mathrm{pixel}$.

The mold used during the potting procedure was $101.6 \mathrm{~mm}$ in diameter. The midpoint of the pot was calculated to be $50.8 \mathrm{~mm}$ from the anterior edge of the pot in the radiograph as shown in Figure 3.3. A line along the top of the potting material was added and a blue dot was placed on this line to mark the midpoint. The pitch axis of the spine robot is located along this central line 0.3825 inches from the top of the pot. Measurements were made from the midpoint defined above and the pitch axis was placed as a large red dot in the figure. All subsequent measurements for the grid were made from this point. The yellow lines in the figure demarcate the anterior and posterior limits of the endplates and assign an orientation to the vertebral bodies. A disc midline was created parallel to the subjacent body endplate, lying approximately centrally between the two endplate lines. An inferior line was also created parallel to the subjacent body endplate but lying $5 \mathrm{~mm}$ below the inferior endplate line. With these landmarks identified, a grid system was then placed on each image.

As discussed in Section 2.4, the literature identifies several distinct regions where 


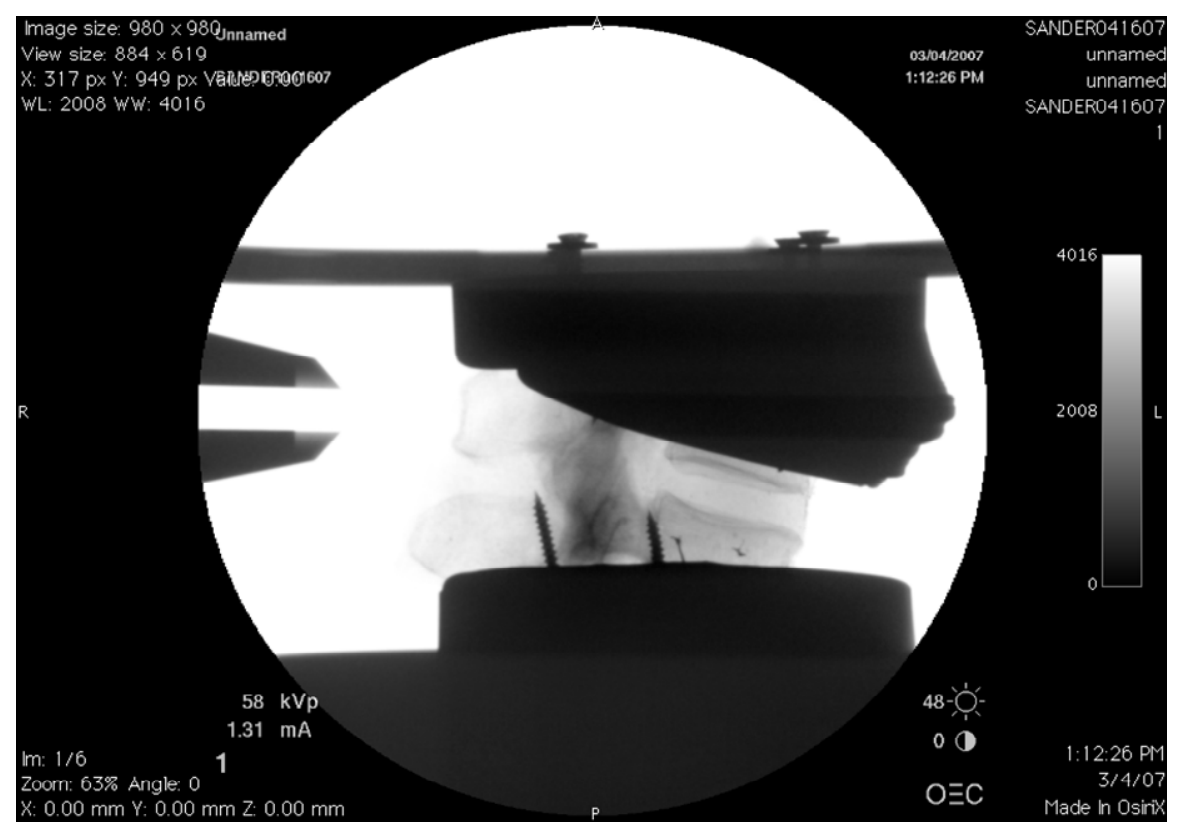

Figure 3.2 Lumbar Specimen with Caliper Set to $10 \mathrm{~mm}$. 


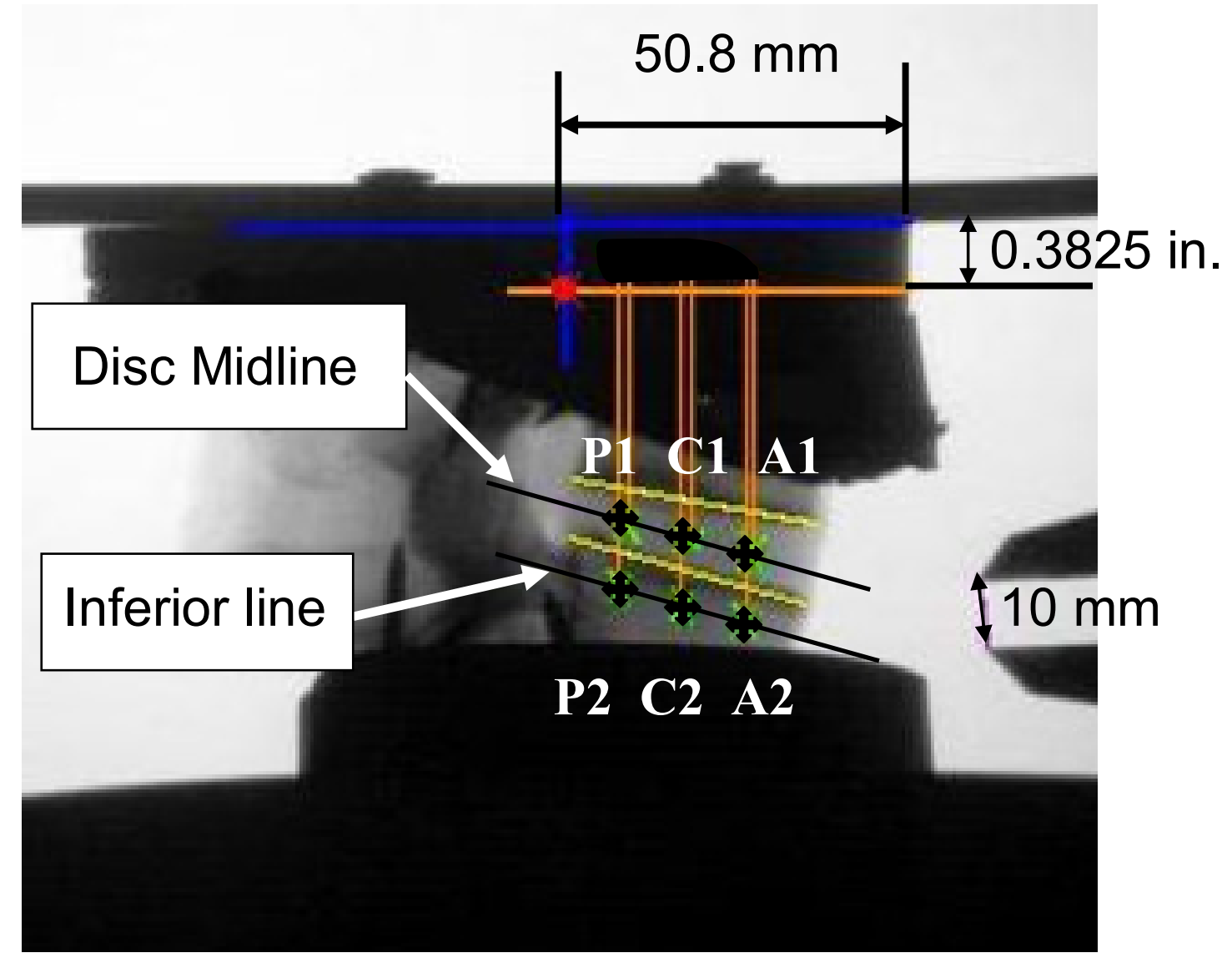

Figure 3.3 Lumbar Segment with Point Grid. 
the physiologic COR is thought to be located. In this protocol, three COR locations were placed in the image related to these regions $(\mathrm{C} 1, \mathrm{P} 1$ and $\mathrm{P} 2)$. The remaining three points (A1, A2, and C2) were placed to provide a more complete analysis of the effects of different COR locations. The six points were marked with small green Xs. Three points were located along the midline of the disc in the A-P direction. $\mathrm{C} 1$ was located at the midpoint of the disc, A1 was halfway between the mid-point and anterior aspect of the disc, and P1 was halfway between the mid-point and posterior aspect of the disc. The remaining three points $(\mathrm{C} 2, \mathrm{~A} 2$, and $\mathrm{P} 2)$ lay on the inferior line, located $5 \mathrm{~mm}$ below the proximal endplate of the subjacent vertebral body, with the same A-P orientations as $\mathrm{C} 1$, $\mathrm{A} 1$, and P1. For each specimen, the horizontal (X) and vertical (Z) distances were measured from the pitch axis to each individual point. These distances are reported in Tables 3.1 and 3.2. Grids for each of the specimens can be found in Appendix A.

\subsection{University of Tennessee Spine Robot}

Testing of each specimen was conducted using a custom designed spine robot which was developed in the Biomechanical Tissue Testing Laboratory at the University of Tennessee Health Science Center. ${ }^{36}$ The spine robot has four controllable DOFs. The robot is shown in Figure 3.4. Table 3.3 shows specifications for each DOF. It has been shown in the lab that the overall positional accuracy of the robot is limited by the individual resolution of each axis. ${ }^{36}$

Figure 3.5 shows the individual DOFs of the robot. In the figure, "A" corresponds to the axis which provides horizontal (X) translation; "B" corresponds to the vertical $(Z)$ translation. For the $3^{\text {rd }}$ and $4^{\text {th }}$ DOFs a gimbal fixture was created to house two rotary axes. This gimbal was mounted directly to the carriage on the horizontal axis. "C" corresponds with the rotary axis responsible for rotation of the spine in either flexion/extension or, if the specimen were rotated 90 degrees, right/left lateral bending. "D" corresponds to the axis which provides an axial rotation of the specimen for potential coupled motion testing. In this study the axial motion was constrained and only flexion/extension in the sagittal plane was allowed. This was done to simplify the motion and force analysis, reduce the programming complexity, and to limit the coupled interactions associated with spinal motion. During the constrained planar motion all outof-plane forces and moments were monitored and analyzed. Constraining motion to a single plane is a common practice in spine biomechanics. Further protocols are currently being developed in the lab which can better account for the more complex, coupled loading conditions.

The robot was controlled using an Adept motion controller (Adept Technologies, San Jose, CA) which is housed in a separate electrical cabinet as seen in Figure 3.4. The cabinet also contains all of the power supplies, amplifiers, and transformers. During construction of the robot, special attention was given to proper grounding of the robot components to reduce any noise in the electrical system. To further reduce interference during testing, all of the external cables were replaced with braided grounding cables. This improved the function of the robot and its overall robustness. 
Table 3.1 $\mathrm{X}$ and $\mathrm{Z}$ Transformation Values for Points A1-P1.

\begin{tabular}{lccccccc}
\hline \multirow{2}{*}{ Specimen \# } & \multicolumn{2}{c}{$\mathrm{A} 1$} & & \multicolumn{2}{c}{$\mathrm{C} 1$} & & \multicolumn{2}{c}{$\mathrm{P} 1$} \\
\cline { 8 - 9 } 0105416 (L3-L4) & 24.2 & -47.5 & 13.1 & -46.9 & 5.8 & -42.5 \\
UJ00D35 (L3-L4) & 28.2 & -36.4 & 17.7 & -41.1 & 8.2 & -37.3 \\
ADS0612051 (L3-L4) & 31.9 & -46.6 & 21.5 & -43.5 & 11.1 & -41.3 \\
ADS0612051 (L5-S1) & 13.6 & -53.6 & 6.4 & -48.6 & 0.0 & -42.9 \\
DRT060069 (L3-L4) & 26.7 & -37.3 & 17.8 & -35.7 & 8.9 & -34.6 \\
DRT050786 (L3-L4) & 13.0 & -41.4 & 5.5 & -38.7 & -3.0 & -36.2 \\
UF01A030 (L3-L4) & 21.6 & -43.6 & 11.5 & -41.5 & 1.62 & -39.7 \\
\hline
\end{tabular}

Table 3.2 X and Z Transformation Values for Points A2-P2.

\begin{tabular}{|c|c|c|c|c|c|c|}
\hline \multirow[b]{2}{*}{ Specimen \# } & \multicolumn{2}{|c|}{$\mathrm{A} 2$} & \multicolumn{2}{|c|}{$\mathrm{C} 2$} & \multicolumn{2}{|c|}{$\mathrm{P} 2$} \\
\hline & $\bar{X}$ & $\mathrm{Z}$ & $\mathrm{X}$ & $\mathrm{Z}$ & $\mathrm{X}$ & $\mathrm{Z}$ \\
\hline 0105416 (L3-L4) & 20.0 & -58.3 & 10.8 & -55.8 & 3.3 & -53.3 \\
\hline UJ00D35 (L3-L4) & 22.7 & -59.1 & 12.7 & -55.5 & 2.7 & -49.1 \\
\hline ADS0612051 (L3-L4) & 30.7 & -56.5 & 20.4 & -54.2 & 11.1 & -50.7 \\
\hline ADS0612051 (L5-S1) & 2.2 & -59.3 & -1.4 & -54.3 & -9.3 & -49.6 \\
\hline DRT060069 (L3-L4) & 25.6 & -46.8 & 16.0 & -45.3 & 7.3 & -44.3 \\
\hline & 12.3 & -50.4 & 4.0 & -48.0 & -4.1 & -45.3 \\
\hline UF01A030 & 20.6 & -55.1 & 10.6 & -52.3 & 0.9 & -50.3 \\
\hline
\end{tabular}




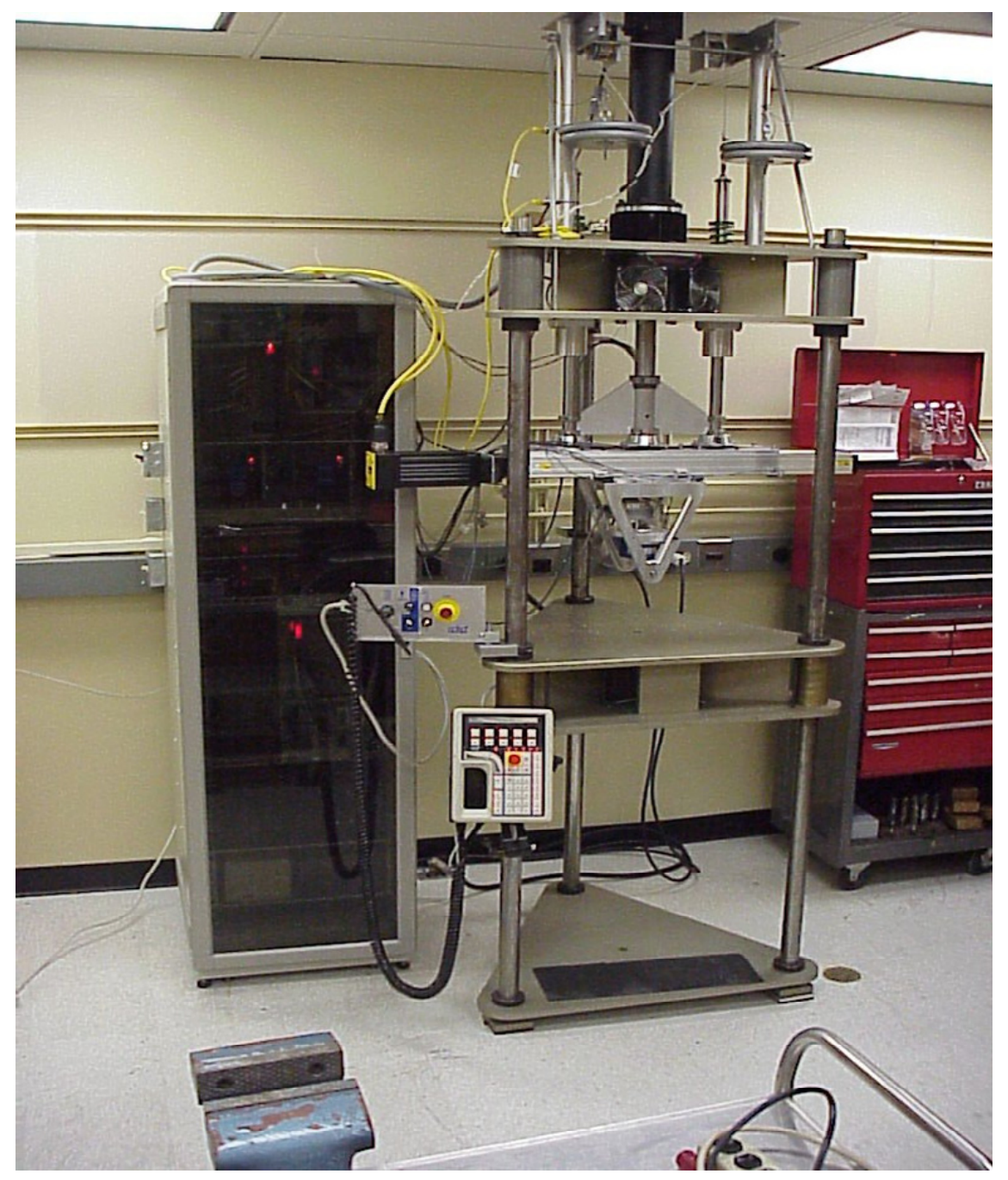

Figure 3.4 UT Spine Robot and Control Cabinet. Reprinted with permission from Bonin H, In-vitro analysis of the instantaneous center of rotation in a human cervical spine model using a spine robot, Thesis, 2006, University of Tennessee Health Science Center. $^{4}$ 
Table 3.3 UT Spine Robot Specifications. Reprinted with permission from Bonin H, In-vitro analysis of the instantaneous center of rotation in a human cervical spine model using a spine robot, Thesis, 2006, University of Tennessee Health Science Center. ${ }^{4}$

\begin{tabular}{|c|c|c|c|c|c|}
\hline DOF & $\begin{array}{c}\text { Mechanism } \\
\text { Type }\end{array}$ & $\begin{array}{c}\text { Device/ } \\
\text { Manufacturer }\end{array}$ & Description & ROM & Resolution \\
\hline 1 & $\begin{array}{c}\text { Linear Ball } \\
\text { Screw } \\
\text { Actuator }\end{array}$ & $\begin{array}{l}\text { 406XR / Parker } \\
\text { Automation }\end{array}$ & $\begin{array}{l}\text { Provides horizontal } \\
\text { translation (x-axis) }\end{array}$ & $600 \mathrm{~mm}$ & $2 \mu \mathrm{m}$ \\
\hline 2 & $\begin{array}{c}\text { Linear Roller } \\
\text { Screw } \\
\text { Actuator }\end{array}$ & $\begin{array}{l}\text { GSX-30 / Exlar } \\
\text { Corporation }\end{array}$ & $\begin{array}{l}\text { Provides vertical } \\
\text { translation (z-axis) }\end{array}$ & $300 \mathrm{~mm}$ & $0.31 \mu \mathrm{m}$ \\
\hline 3 & $\begin{array}{c}\text { Rotary } \\
\text { Geared } \\
\text { Servomotor }\end{array}$ & $\begin{array}{c}\text { 9FG / } \\
\text { Kollmorgen } \\
\text { PMI Division }\end{array}$ & $\begin{array}{l}\text { Provides rotation } \\
\text { about the } y \text {-axis in } \\
\text { the } x-z \text { plane }\end{array}$ & $\begin{array}{c}360 \\
\text { degrees }\end{array}$ & $\begin{array}{l}0.0045 \\
\text { degrees }\end{array}$ \\
\hline 4 & $\begin{array}{c}\text { Rotary } \\
\text { Geared } \\
\text { Servomotor }\end{array}$ & $\begin{array}{c}\text { 9FG / } \\
\text { Kollmorgen } \\
\text { PMI Division }\end{array}$ & $\begin{array}{l}\text { Provides rotation } \\
\text { about the } z \text {-axis in } \\
\text { the } x-y \text { plane }\end{array}$ & $\begin{array}{c}360 \\
\text { degrees }\end{array}$ & $\begin{array}{l}0.0045 \\
\text { degrees }\end{array}$ \\
\hline
\end{tabular}




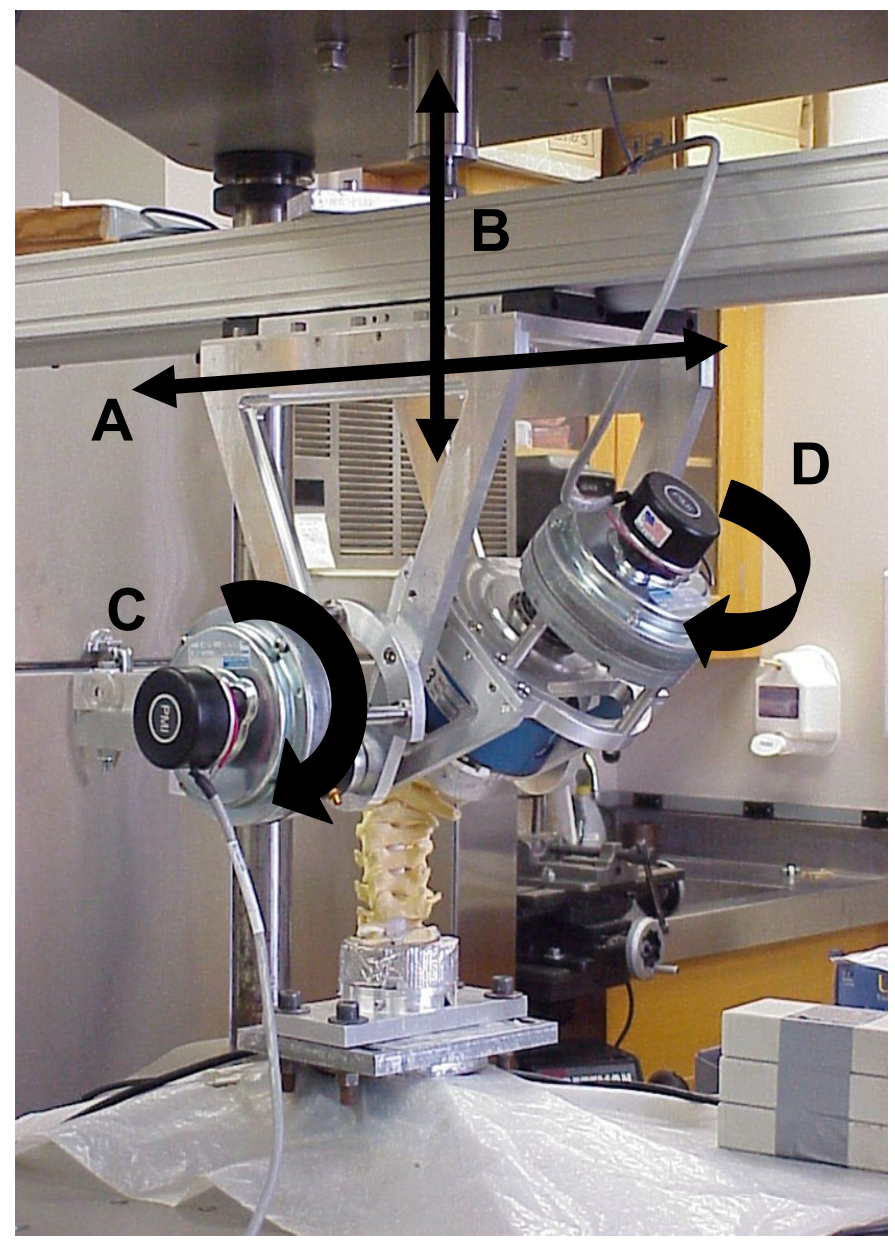

Figure 3.5 Four Degrees of Freedom of the UT Spine Robot. A and B provide horizontal and vertical translation, $\mathrm{C}$ is rotation about the $\mathrm{Y}$ axis, and $\mathrm{D}$ is axial rotation about the $\mathrm{Z}$ axis. 
The Adept controller utilizes an Adept programming language called V+. For each of the protocols utilized with the robot, a new custom program was created. These programs were written in the $\mathrm{V}+$ language and can be found in Appendix B.

\subsection{Kinematics-Based Testing Protocol}

A new testing protocol has been proposed that involves prescribing a known kinematic input to a single spinal MSU and measuring the capacity of the intact MSU to accommodate the motion. Panjabi described this type of protocol as stiffness or displacement input protocol. ${ }^{44}$ Using the spine robot, the kinematic profile of an intact MSU can be programmed to follow a specified path or to rotate about a fixed point in space. For the kinematics protocol, the grid points described in Section 3.1.3 were used as fixed CORs. Pure rotations were prescribed about each of these points. Figure 3.6 shows how the robot accommodates a pure rotation about the prescribed points. In order to rotate about the defined COR the robot has to translate along the $\mathrm{X}$ and $\mathrm{Z}$ axes in conjunction with pure rotation of the pitch axis. This is accomplished by using the robot's built-in transformation function and trajectory planner.

Prior to testing, the specimens were thawed overnight in a refrigerated environment. Each specimen was aligned in the robot so that the pitch axis on the radiographs coincided with the initial tool position of the robot. This initial tool position is a calibrated point in Cartesian space that is known by the robot. Since the robot was originally developed for use in an industrial setting, this point coincides with the tool tip or end effectors of the robot. Adept built a transformation function into its controller to allow industrial users to attach different end effectors (i.e. grippers, hydraulic presses, multi-length manipulators, etc.) and reprogram the initial tool position to coincide with each new tool tip. Once a new tool tip had been specified, the controller, using a trajectory planner, defined the appropriate trajectory and commanded the individual axes to move with respect to the new point. This transformation, in our application, was used to specify rotation about the COR grid points.

Figure 3.7 shows the lines of code used to specify the tool transformation. Line 1.3 allows a user to input a tool transformation coordinate in the $\mathrm{X}$ axis and Line 1.5 is the transformation in the $\mathrm{Z}$ axis. These transformations were previously measured from the radiographs with respect to the robot's world coordinate system (WCS). Line 1.6 introduced a variable that would maintain these new tool transformation coordinates. Line 1.7 then changed the robot's initial tool position to these newly established coordinates. From that point forward all subsequent move commands were performed with respect to the new tool position. Line 1.8 reset the force frame of reference from its initial position to coincide with the new tool tip position. This was done so that all moment measurements were made with respect to the COR and not the initial tool position.

A $20 \mathrm{~N}$ axial preload was applied to the specimen prior to initiating any move command. The specimen was allowed to carry the full weight of the upper potting 


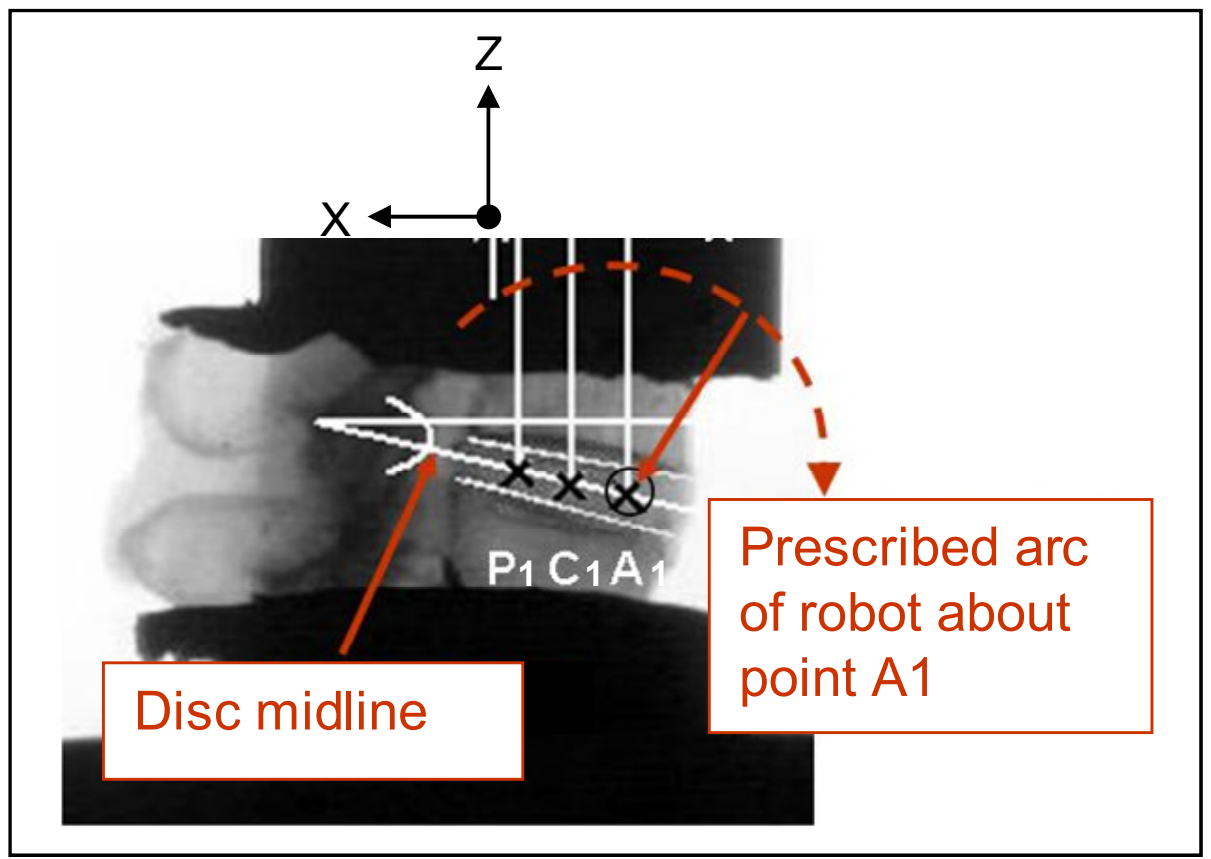

Figure 3.6 Arc of Rotation Prescribed by Robot. 


\begin{tabular}{|c|c|}
\hline 1.1 & TYPE /X20, "****TOOL TRANSFORMATION INFORMATION*****" \\
\hline 1.2 & TYPE $/ \mathrm{C} 2$ \\
\hline 1.3 & $\begin{array}{l}\text { PROMPT "What is the value of your tool transformation in the WCS } x \text { direction?: } \\
\text { ", xvalue }\end{array}$ \\
\hline 1.4 & $\mathrm{TYPE} / \mathrm{C} 1$ \\
\hline 1.5 & $\begin{array}{l}\text { PROMPT "What is the value of your tool transformation in the WCS z direction? } \\
\text { (note: this should be negative): ", zvalue }\end{array}$ \\
\hline 1.6 & $\begin{array}{l}\text { SET this.point }=\text { TRANS(xvalue, ,-zvalue) } ;(-) \text { zvalue to cancel the (-) entered } \\
\quad \text { in prompt - for our system this must be positive }\end{array}$ \\
\hline 1.7 & TOOL this.point \\
\hline 1.8 & FORCE.FRAME (1) TRANS(-xvalue, ,zvalue-51.549) \\
\hline
\end{tabular}

Figure 3.7 Adept V+ Code Used to Set Tool Transformation. Reprinted with permission from Bonin $\mathrm{H}$, In-vitro analysis of the instantaneous center of rotation in a human cervical spine model using a spine robot, Thesis, 2006, University of Tennessee Health Science Center. ${ }^{4}$ 
material and fixation hardware and then the remaining load was applied by the vertical actuator. Since each of the specimens had an upper pot of varying weight, each pot was weighed individually prior to the start of testing. This was accomplished by supporting the weight of the spine and lower pot and suspending the mass of the upper pot from a six axis load cell. The force exerted on the load cell by the weight of the upper potting material was measured and input into the custom software program. The remaining preload was then applied using the "spine.preload23" program (Appendix B.3). So, for example, if the pot weight was $16 \mathrm{~N}$ then the spine.preload 23 program would apply an additional $4 \mathrm{~N}$ for a $20 \mathrm{~N}$ total preload on the specimen.

End limits of motion, moment, and force were established using a guarded trigger function built into the Adept controller. This function allowed the user to input a moment and/or force value after which the robot would execute all move commands until one of the limits was triggered. At that point the robot stopped all motions immediately. The motion end limit was set according to the recommendations from the disability index used by the American Medical Association (AMA). ${ }^{8}$ The AMA suggests that a normal lumbar spine, from $\mathrm{T} 12$ to $\mathrm{S} 1$, should have at least 60 degrees of motion in flexion. Using this guideline the motion end limit was set at 12 degrees of rotation. The moment and force end limits were $8 \mathrm{Nm}$ and $500 \mathrm{~N}$ respectively. It should be noted that the moment and force limits were three dimensional resultant values with respect to all three axes (X, Y, and Z). These limits were non-destructive and acted as protection against damaging the tissue. The $8 \mathrm{Nm}$ moment end limit also functioned as the common load at which all analysis was completed.

All testing on a specimen was completed in the same day and a saline solution was used to keep the tissue from dehydrating. Testing was performed for each specimen, about each grid point, in the following order: A1, C1, P1, A2, C2, and P2. Flexion testing was first completed for each point, followed by extension testing in the same sequence. All tests proceeded at a constant rotational velocity of 1.2 degrees/second until one of the end limits was reached.

\subsection{Data Management and Analysis}

\subsubsection{Data Collection}

The motion of each axis of the robot was recorded by the controller relative to the WCS. All transformations in the coordinate systems were handled by the program written for this test (fixed_axis1). During the tests, the following were measured for each trajectory cycle: time; forces and relative motion of the $\mathrm{X}, \mathrm{Y}$, and $\mathrm{Z}$ axes; and moments about the $\mathrm{X}, \mathrm{Y}$, and $\mathrm{Z}$ axes. The program used a proprietary code from Adept called "buf.fs.v2" to gather all of the data. A secondary custom program, which had been previously written, called "write_to_file23" was used to extract the buffer data and export it to a .txt file on the computer. Figure 3.8 shows the sign convention for forces, moments, and rotations in the data after the transformations had been applied. Positive 


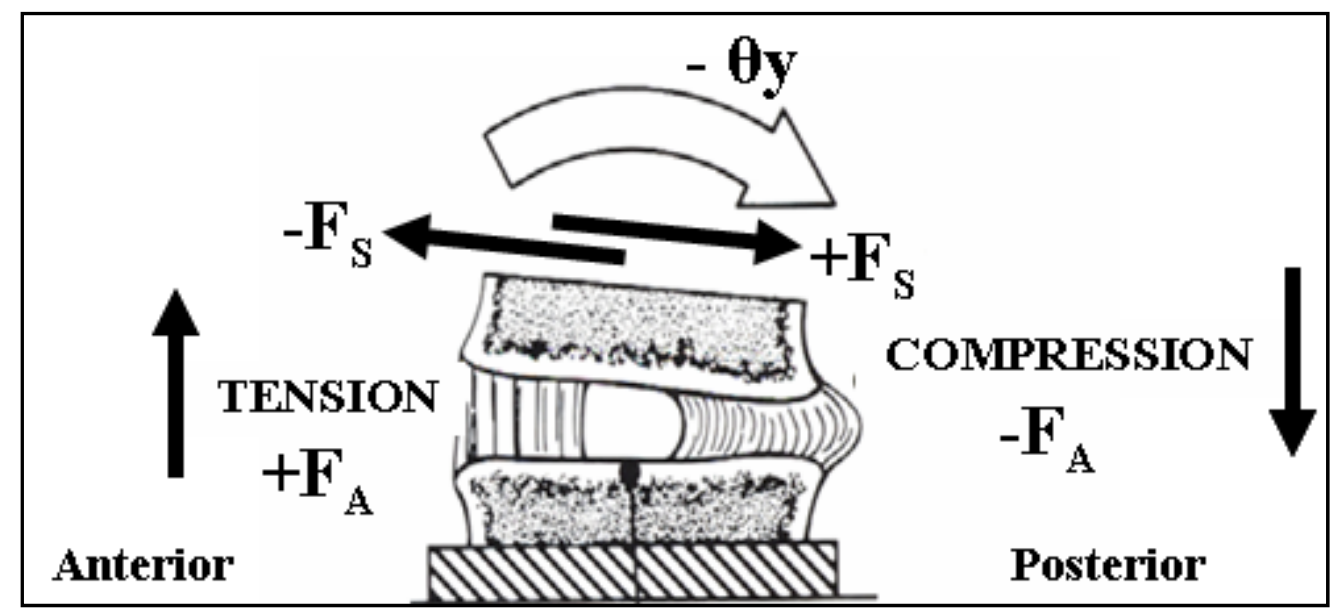

Figure 3.8 Sign Convention of Test Results. Adapted with permission from Bonin $\mathrm{H}$, In-vitro analysis of the instantaneous center of rotation in a human cervical spine model using a spine robot, Thesis, 2006, University of Tennessee Health Science Center. ${ }^{4}$ 
MSU axial force normal to the disc plane $\left(+\mathrm{F}_{\mathrm{A}}\right)$ indicated net MSU tissue tension, and negative MSU axial force $\left(-\mathrm{F}_{\mathrm{A}}\right)$ indicated net MSU tissue compression. Positive A$\mathrm{P}$ shear force along the disc plane $\left(+\mathrm{F}_{\mathrm{S}}\right)$ indicated a net MSU posterior shear, and negative A-P shear force $\left(-\mathrm{F}_{\mathrm{S}}\right)$ indicated a net MSU anterior shear. Positive sagittal rotation $(+\theta y)$ indicated flexion, and negative sagittal rotation $(-\theta y)$ indicated extension.

Force measurements were made using a six axis load sensor (Model 100M40, JR3 Incorporated, Woodland, CA). The load sensor was attached to the pot of the top, unconstrained vertebral body and made the connection to the robot. The original coordinate system was centered in the load cell body, but using the Adept controller this coordinate system was transformed so as to coincide with the COR for each test, as described in Section 3.3.1. The load cell is shown in Figure 3.9. It had a load capacity of $890 \mathrm{~N}$ in the $\mathrm{Z}$ axis, $445 \mathrm{~N}$ in the $\mathrm{X}$ and $\mathrm{Y}$ axes, and $45.2 \mathrm{Nm}$ about all axes.

\subsubsection{Statistical Analysis}

For data analysis a common moment end limit of $8 \mathrm{Nm}$ was used. Force and location data at the $8 \mathrm{Nm}$ limit were collected, separated into flexion and extension data, and organized into the Excel Spreadsheet (Microsoft, Redmond, WA) shown in Appendix C. The data from each specimen were used to calculate a mean value for each of the important parameters. Analysis was conducted on mean axial forces, mean shear forces, and mean range of motion (rotation).

For statistical analysis, the data was copied into SigmaStat 3.5 (Systat Software Inc., San Jose, CA) and indexed for analysis. Figure 3.10 shows a screen shot from a SigmaStat data sheet with a typical indexing example used in the analysis. The data was processed and statistically analyzed as part of a broader study; however, the harvested data will only be considered for the scope of this study and are presented in the following sections.

Initial normality tests of the data indicated non-normal distributions for all data sets which resulted in a statistical ranking being applied to each data set individually. Two-Way, Repeated Measures Analysis of Variance (ANOVA) tests were performed to calculate significance between grid points in the axial force, shear force, and rotation data for all specimens $(\mathrm{p}=0.05)$. A Student-Newman-Keuls (SNK) test was used to find significance between all condition and grid point pairs. Statistical comparisons were made between all of the different point pairs both in-plane and inter-plane. In this study, the term "in-plane comparisons" refers to those comparisons made between points on the same plane. This includes the midline comparisons (A1 and C1, A1 and P1, and $\mathrm{C} 1$ and $\mathrm{P} 1$ ) and inferior line comparisons (A2 and $\mathrm{C} 2, \mathrm{~A} 2$ and $\mathrm{P} 2$, and $\mathrm{C} 2$ and $\mathrm{P} 2$ ). The term "inter-plane comparisons" refers to those comparisons made between points on different planes. This includes $\mathrm{A} 1$ and $\mathrm{A} 2, \mathrm{C} 1$ and $\mathrm{C} 2$, and $\mathrm{P} 1$ and $\mathrm{P} 2$. All other inter-plane pairs were tested, but since the in-plane comparisons show the effect of a shift in the COR in the A-P direction, these other inter-plane comparisons were not reported in this study. 


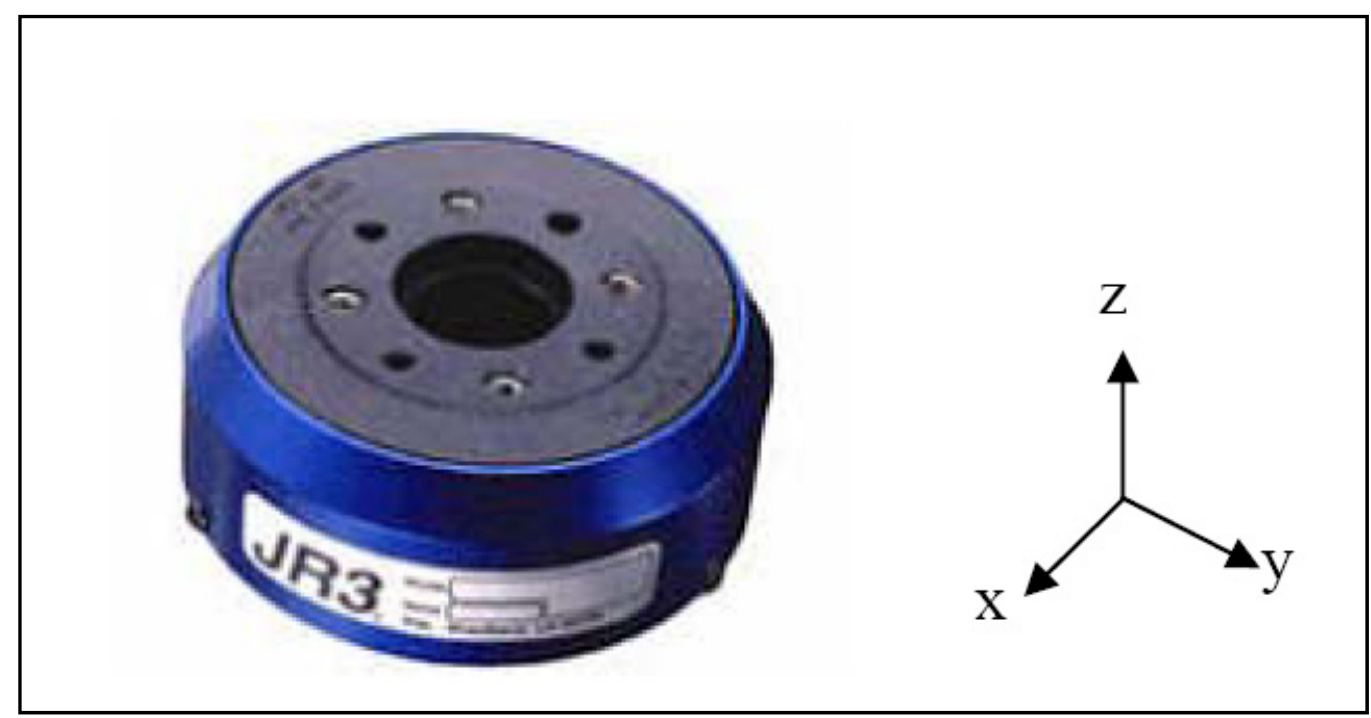

Figure 3.9 Six Axis Load Cell. 


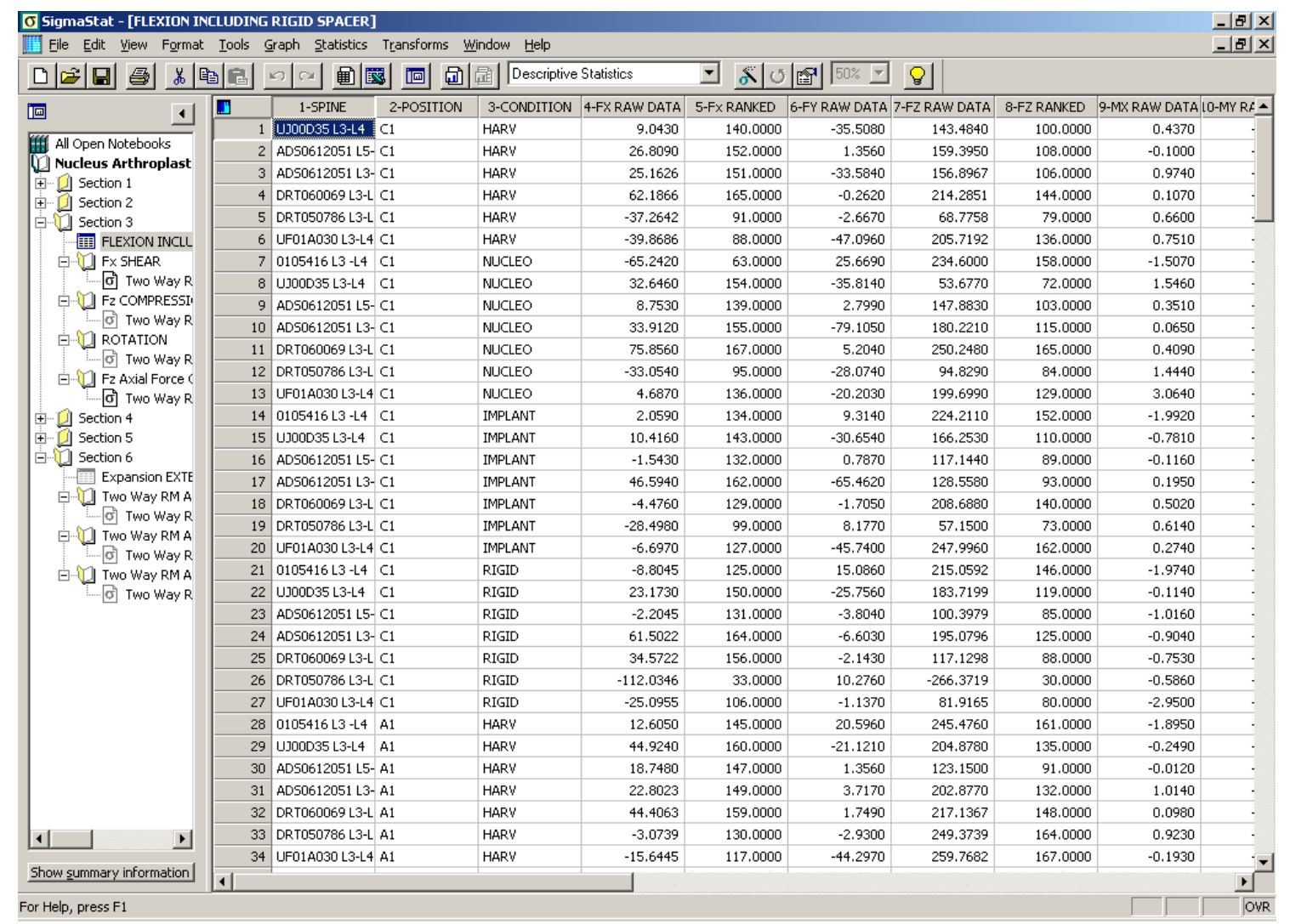

Figure 3.10 SigmaStat Screen Shot. The harvested data set was indexed according to specimen, location of COR, and condition for statistical analysis. 


\section{Chapter 4: Results}

The kinematics-based testing protocol was used to measure the shear and axial force, rotation, and bending moment of seven intact harvested lumbar MSU segments. The results are presented below. The results chapter is divided into two sections. Section one covers shear and axial force results and the second section discusses the rotation results.

\subsection{Load Data}

Typical force and bending moment curves for the kinematics-based testing protocol are presented in Figure 4.1 for specimen UJ00D35 L3-L4 in flexion. All curves are typical and representative of all specimens in flexion and extension. Figure 4.1 (A-F) shows the force versus displacement curves and moment versus displacement curves for all six points of rotation (A1-P1). In the force versus displacement graphs axial force is shown in yellow, A-P shear force is shown in blue, and out-of-plane shear force is shown in pink. In the bending moment versus displacement graphs sagittal bending moment is shown in pink, the axial bending moment is shown in yellow, and the off axis moment is shown in blue. By constraining the motion to sagittal rotation, the off-axis loads and bending moments are theoretically zero, but due to misalignment of the potted specimen and variability in the cadaveric tissue there is a small build-up of off-axis loads. The offaxis loads were not a significant source of error in the study but were monitored to protect the integrity of the tissue.

Figures 4.2-4.5 show the axial and shear force results for flexion and extension rotation. Figures 4.2 and 4.3 show mean axial force data, while Figures 4.4 and 4.5 show mean shear force data. The $\mathrm{X}$ axis in all of the figures represents the different $\mathrm{COR}$ locations (A1-P2). In the axial force graphs, the $\mathrm{Y}$ axis shows axial force in Newtons with positive $\mathrm{F}_{\mathrm{A}}$ representing net tissue tension and negative $\mathrm{F}_{\mathrm{A}}$ representing net tissue compression. In the shear force graphs the $\mathrm{Y}$ axis shows force in Newtons. Positive $\mathrm{F}_{\mathrm{S}}$ indicates a net posterior oriented shear and negative $F_{S}$ indicates a net anterior oriented shear. See Figure 3.8 for further details of the sign convention of all results. Tabulated data used to create the figures for load and motion data can be found in Appendix Tables C.1 and C.2.

\subsubsection{Axial Force}

Figure 4.2 shows the mean axial force results for forward flexion. Rotation about the anterior points $\mathrm{A} 1$ and $\mathrm{A} 2$ resulted in net tissue tension of $214.7 \pm 46.2 \mathrm{~N}$ and $181.0 \pm$ $57.3 \mathrm{~N}$, respectively. For points $\mathrm{C} 1$ and $\mathrm{C} 2$ the tensile loads were slightly lower; however, rotation about the posterior points $\mathrm{P} 1$ and $\mathrm{P} 2$ resulted in net tissue compression of $-251.6 \pm 157.6 \mathrm{~N}$ and $-313.0 \pm 106.3 \mathrm{~N}$, respectively. When comparing points along a plane, the axial forces were significantly different $(\mathrm{p}<0.001)$ between the anterior and 
Figure 4.1 Force and Moment versus Displacement Graphs. Typical graphs for specimen UJ00D35 in flexion for each point of rotation. (A.) Graphs for point A1, (B.) Graphs for point C1, (C.) Graphs for point P1, (D.) Graphs for point A2, (E.) Graphs for point C2, (F.) Graphs for point P2. 

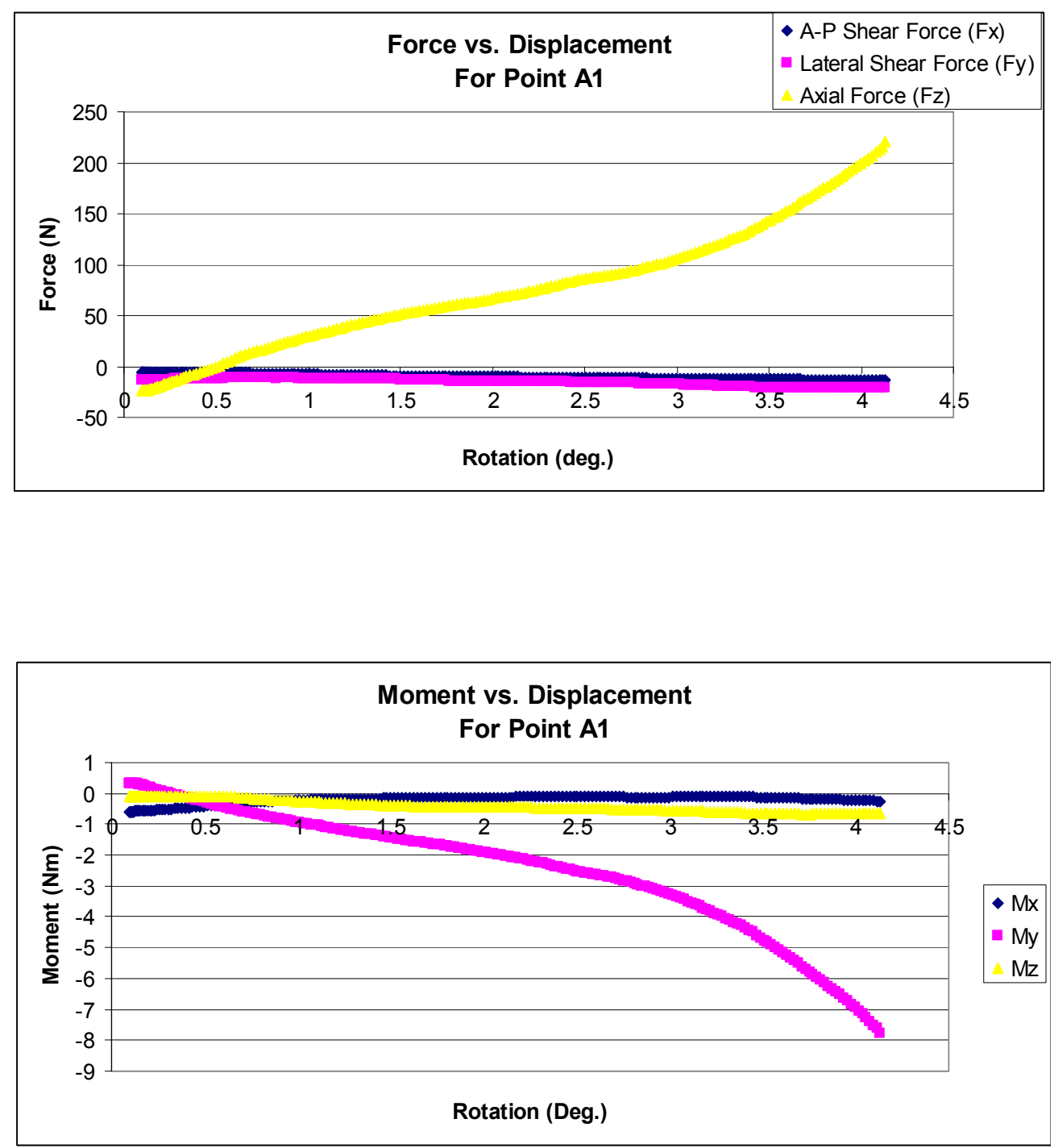

(A.) 

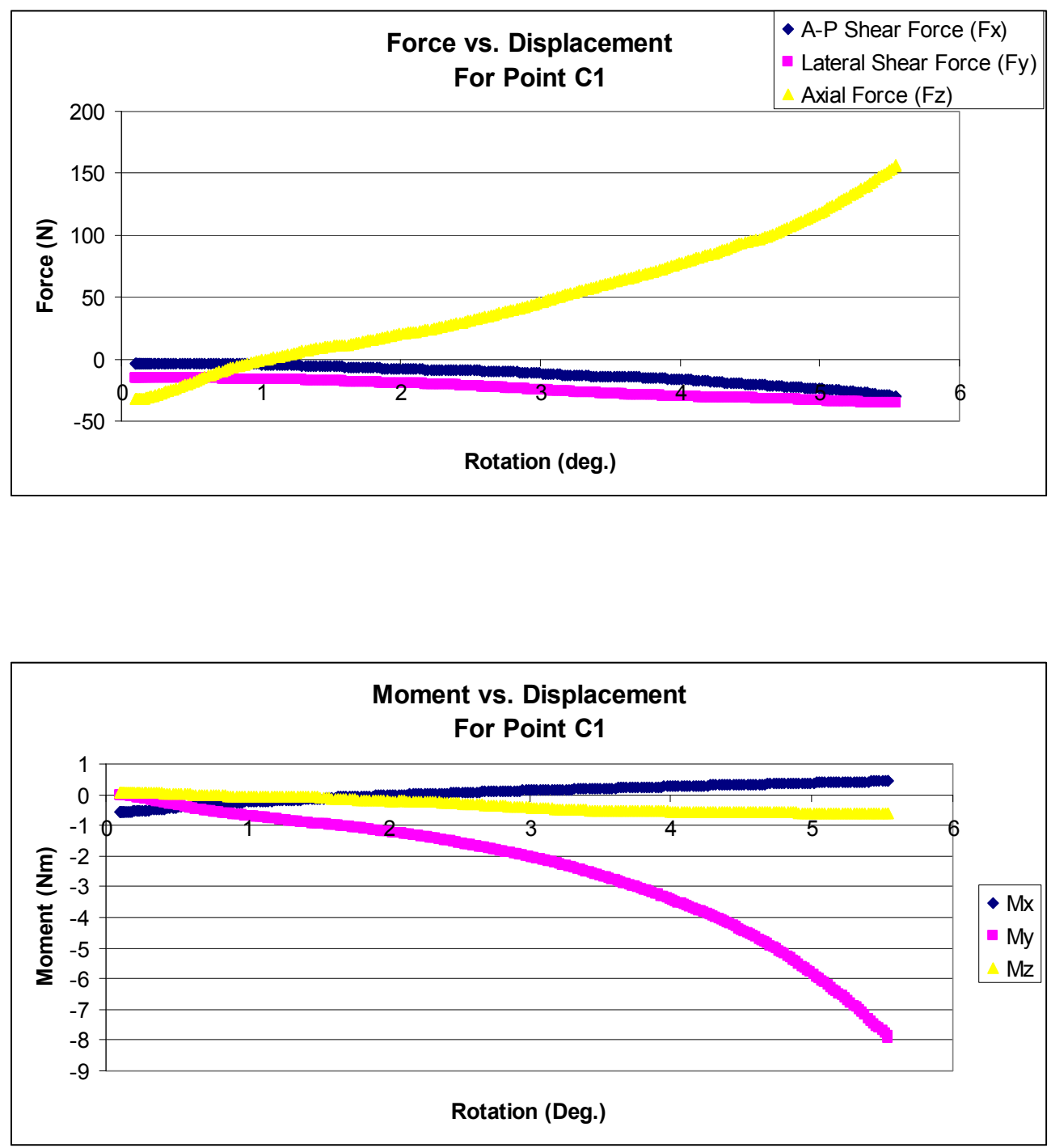

(B.)

Figure 4.1 (Continued). 

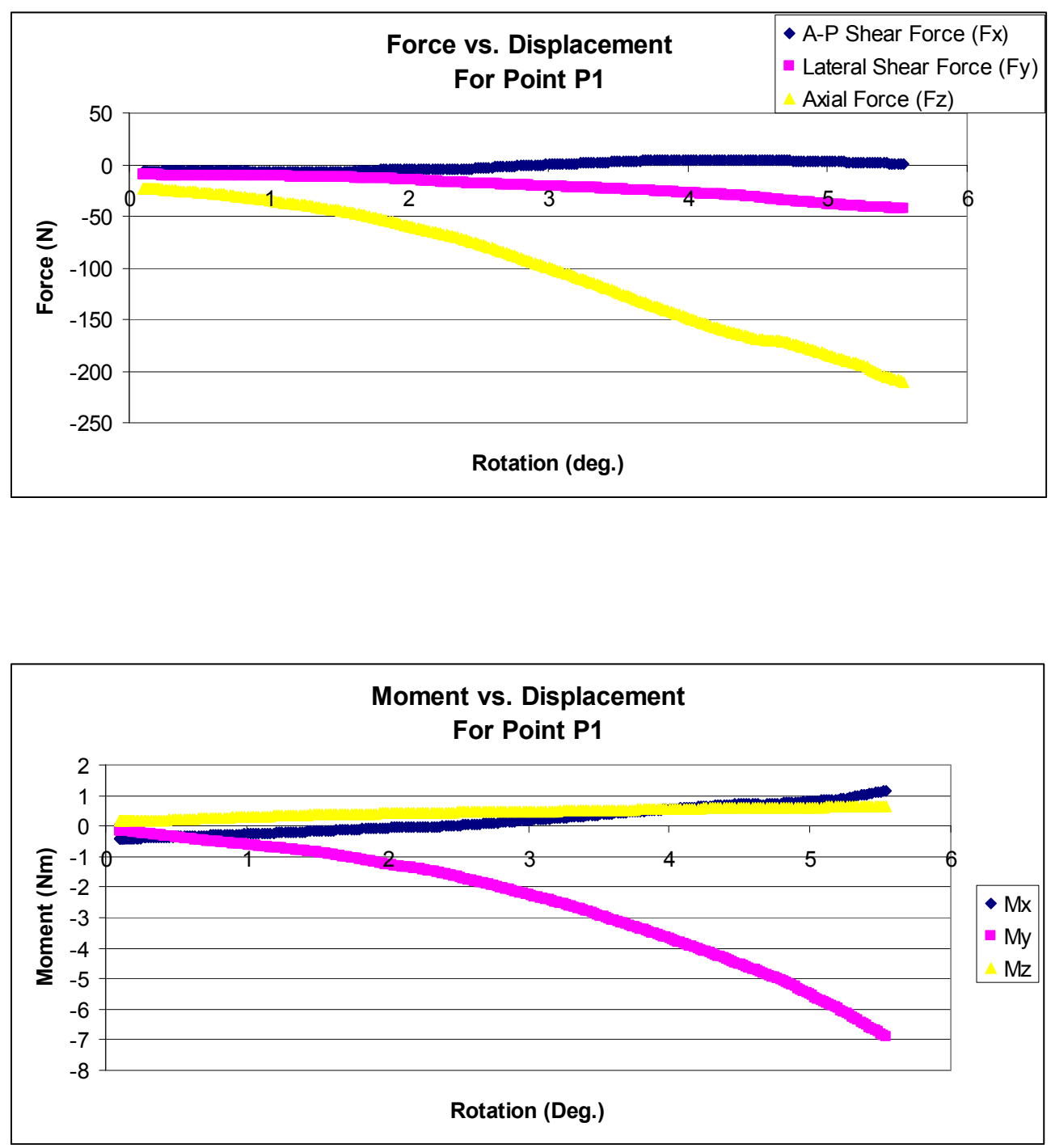

(C.)

Figure 4.1 (Continued). 

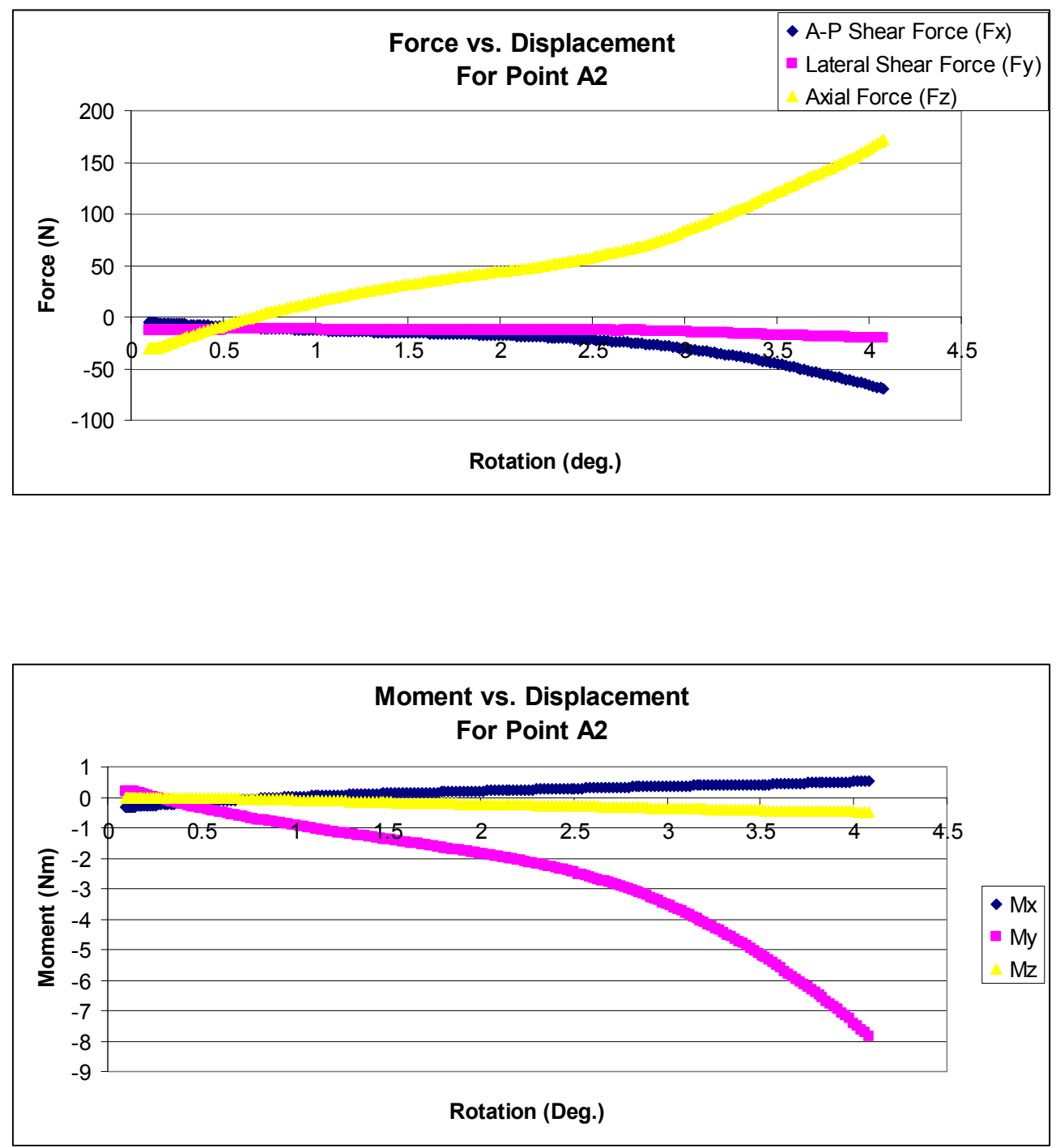

(D.)

Figure 4.1 (Continued). 

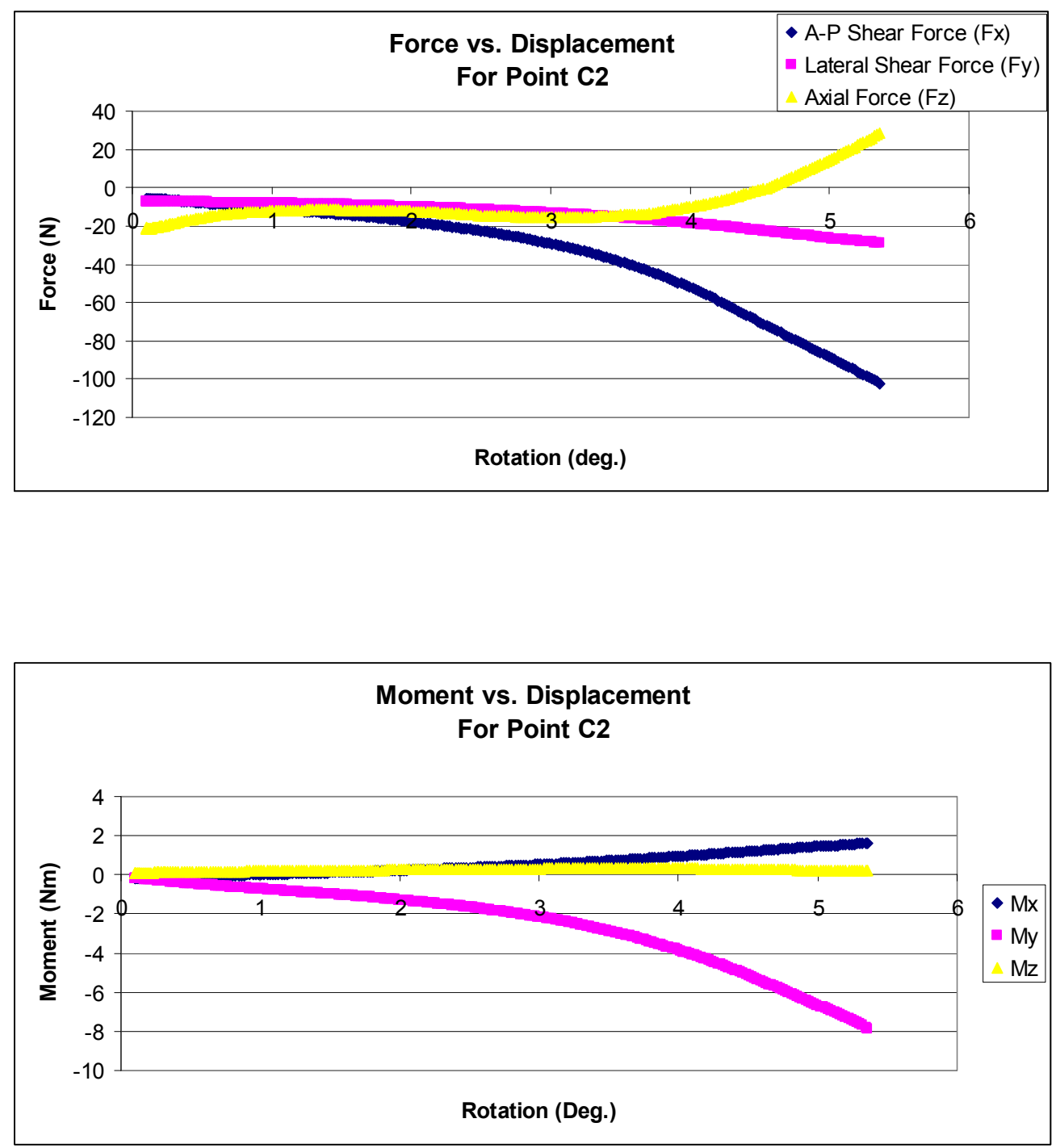

(E.)

Figure 4.1 (Continued). 

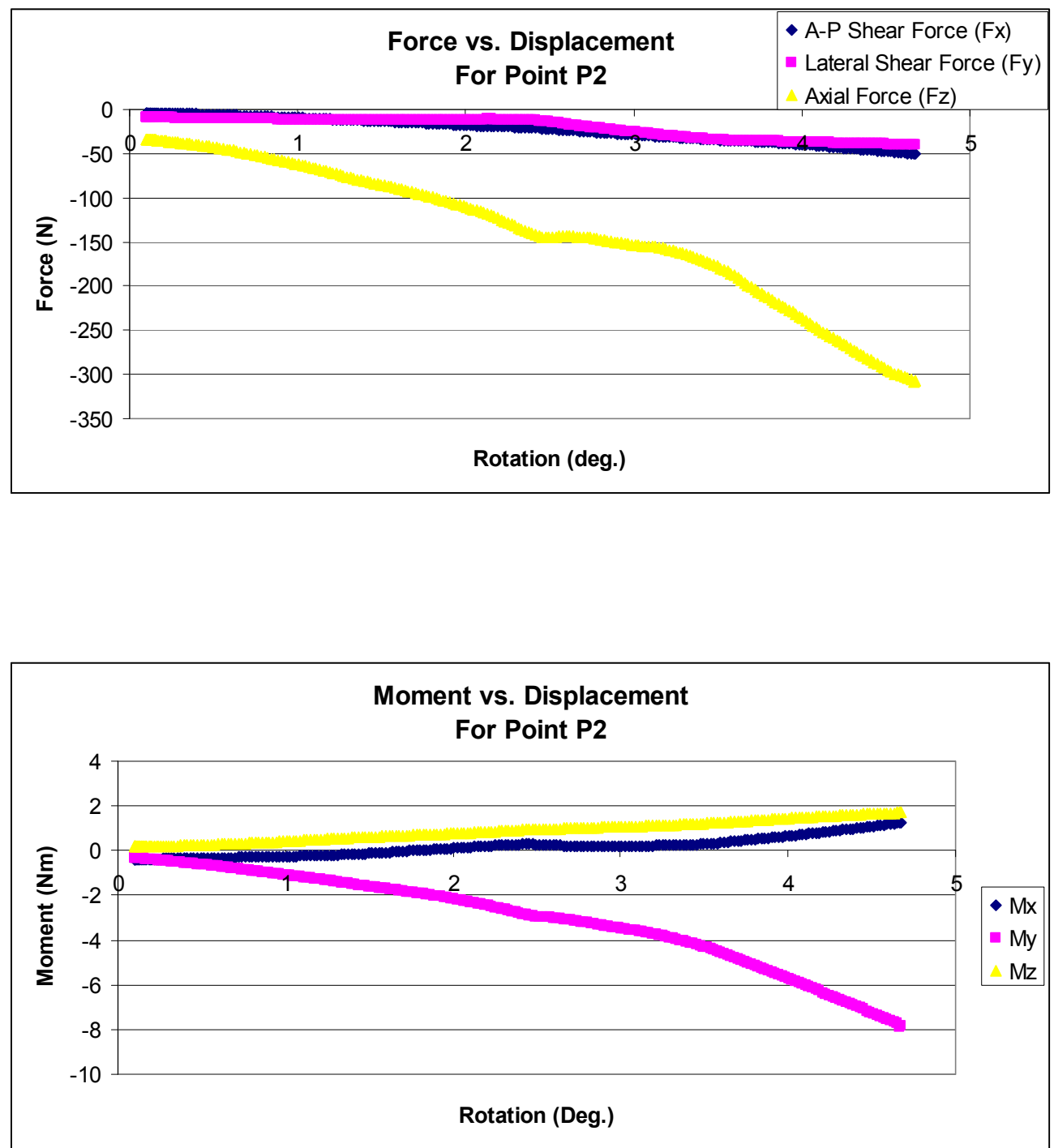

(F.)

Figure 4.1 (Continued). 


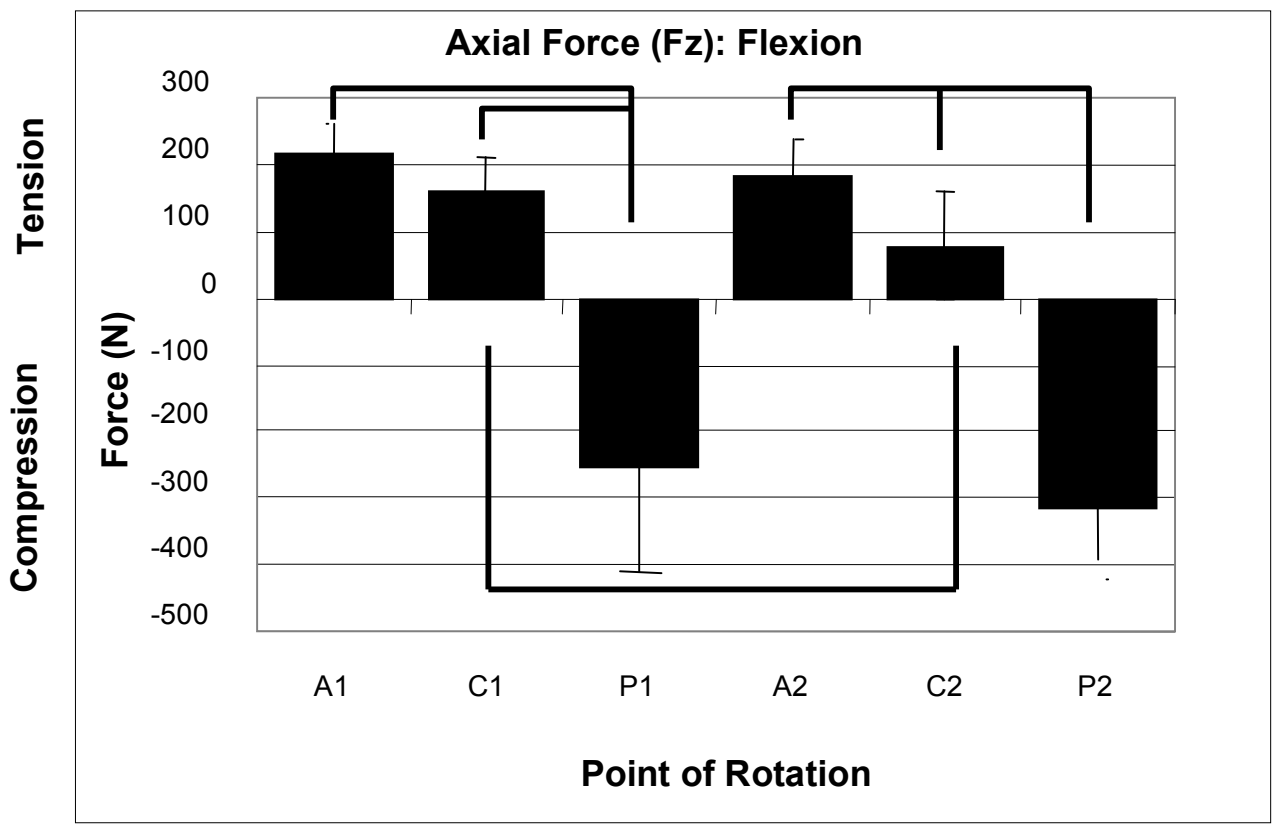

Figure 4.2 Mean Axial Forces for Flexion Rotation.

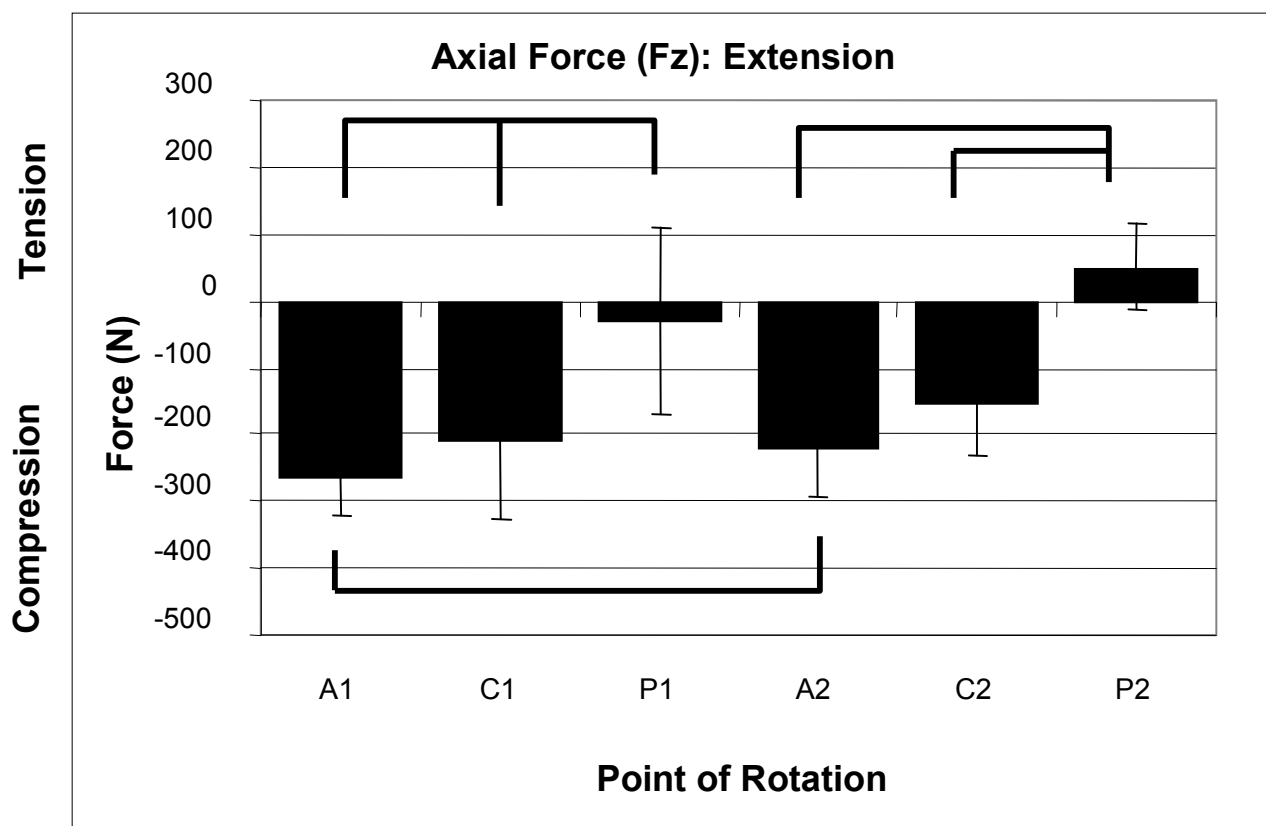

Figure 4.3 Mean Axial Forces for Extension Rotation. 


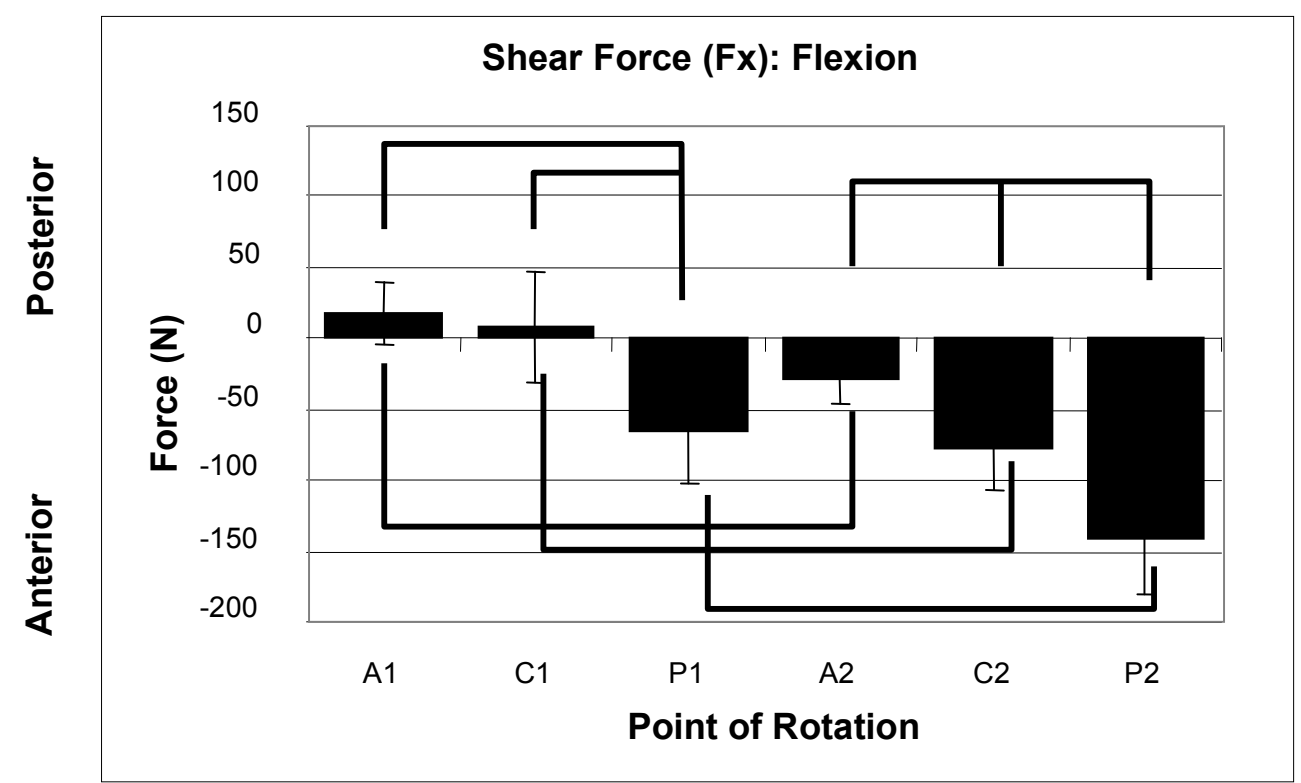

Figure 4.4 Mean Shear Forces for Flexion Rotation.

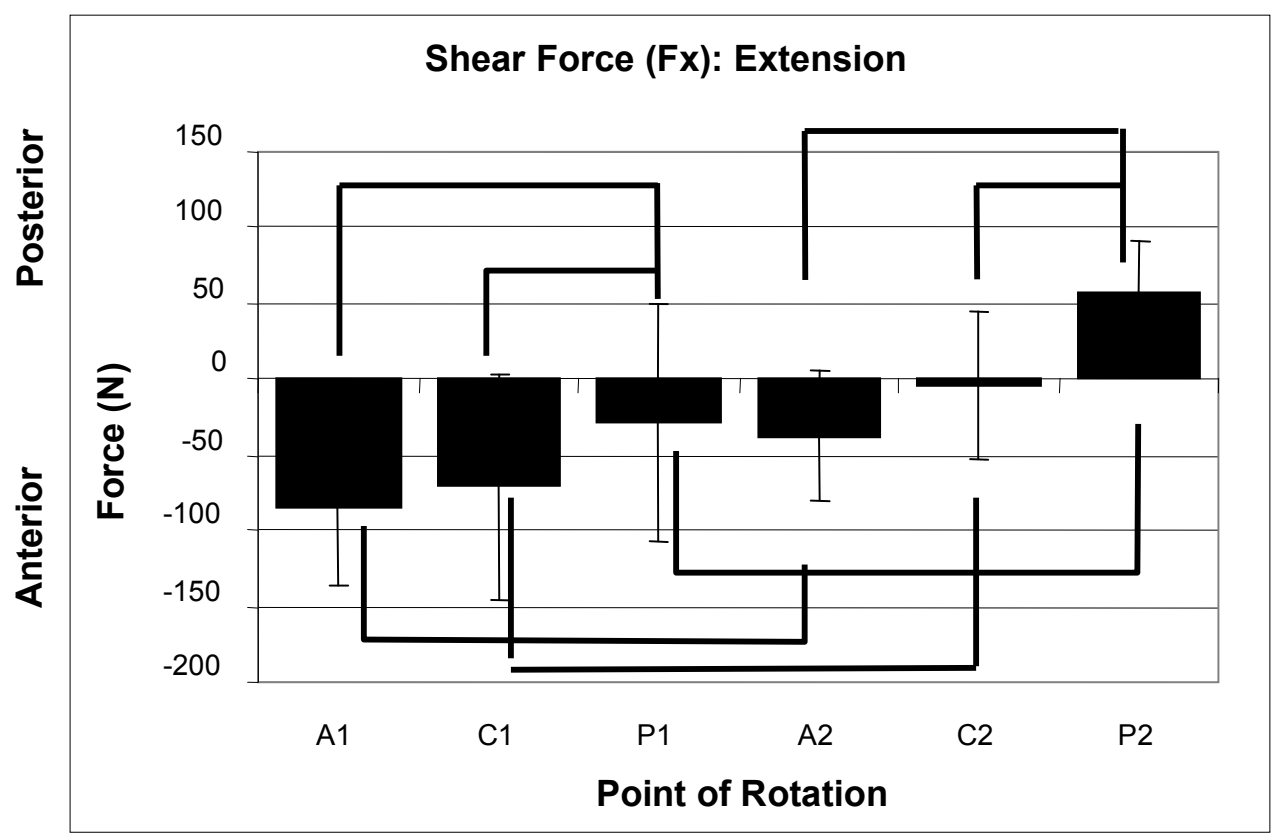

Figure 4.5 Mean Shear Forces for Extension Rotation. 
posterior points and the central and posterior points, but not between the anterior and central points. When comparing points between planes, only $\mathrm{C} 1$ and $\mathrm{C} 2$ were significantly different $(\mathrm{p}=0.04)$.

Mean axial force results for extension rotation are shown in Figure 4.3. The results were nearly opposite in sign to flexion rotation. Rotation about the anterior points $\mathrm{A} 1$ and A2 resulted in net tissue compression of $-263.5 \pm 57.0 \mathrm{~N}$ and $-220.7 \pm 70.1 \mathrm{~N}$, respectively. Rotation about points $\mathrm{C} 1$ and $\mathrm{C} 2$ resulted in lower tissue compression. Points P1 and P2 had either a very low net tissue compression or a slight tissue tension load. With respect to in-plane comparisons, the axial forces were significantly different $(\mathrm{p}<0.001)$ between all points except A2 and C2. Inter-plane, only A1 and A2 were significantly different $(\mathrm{p}=0.05)$.

\subsubsection{Shear Force}

The mean shear force for forward flexion rotation is shown in Figure 4.4.

Rotation about the anterior points A1 and A2 resulted in low shear values of $17.8 \pm 22.5$ $\mathrm{N}$ and $-28.0 \pm 17.6 \mathrm{~N}$, respectively. The mean shear force for rotation about the central point $\mathrm{C} 1$ was $7.7 \pm 39.8 \mathrm{~N}$, but the subjacent central point $\mathrm{C} 2$ had a much higher net anterior oriented shear of $-78.6 \pm 28.6 \mathrm{~N}$. The posterior points $\mathrm{P} 1$ and $\mathrm{P} 2$ had net anterior oriented shear values of $-65.4 \pm 35.6 \mathrm{~N}$ and $-141.5 \pm 38.3 \mathrm{~N}$, respectively. When comparing points along a plane, the shear forces were significantly different $(\mathrm{p}<0.001)$ between the anterior and posterior points and the central and posterior points. When comparing points between planes, all of the points were significantly different $(\mathrm{p}<$ $0.001)$.

Figure 4.5 shows the mean shear force results for extension rotation. Rotation about the anterior points $\mathrm{A} 1$ and $\mathrm{A} 2$ resulted in net anterior oriented shear values of -83.8 $\pm 53.3 \mathrm{~N}$ and $-37.2 \pm 43.5 \mathrm{~N}$, respectively. The mean shear forces for rotation about the central points $\mathrm{C} 1$ and $\mathrm{C} 2$ were $-71.0 \pm 75.4 \mathrm{~N}$ and $-3.5 \pm 49.3 \mathrm{~N}$. The shear forces of the posterior points $\mathrm{P} 1$ and $\mathrm{P} 2$ were $-29.1 \pm 78.6 \mathrm{~N}$ and $56.3 \pm 35.7 \mathrm{~N}$, respectively. Significant differences occurred for points A1 and P1 $(p<0.001), C 1$ and P1 $(p=0.007)$, $\mathrm{A} 2$ and $\mathrm{P} 2(\mathrm{p}<0.001)$, and $\mathrm{C} 2$ and $\mathrm{P} 2(\mathrm{p}<0.001)$ for the in-plane comparisons, and between all points for the inter-plane comparisons.

\subsection{Motion Data}

Figures 4.6 and 4.7 show mean rotation data. In the rotation graphs the $\mathrm{Y}$ axis shows degrees of rotation with positive $\theta_{\mathrm{y}}$ representing flexion rotation and negative $\theta_{\mathrm{y}}$ representing extension rotation. The $\mathrm{X}$ axis in all of the figures represents the different COR locations (A1-P2).

In flexion, rotation about the anterior points A1 and A2 was $3.9 \pm 0.4$ degrees and $4.0 \pm 0.8$ degrees, respectively (Figure 4.6). Rotation about the central points $\mathrm{C} 1$ and $\mathrm{C} 2$ 


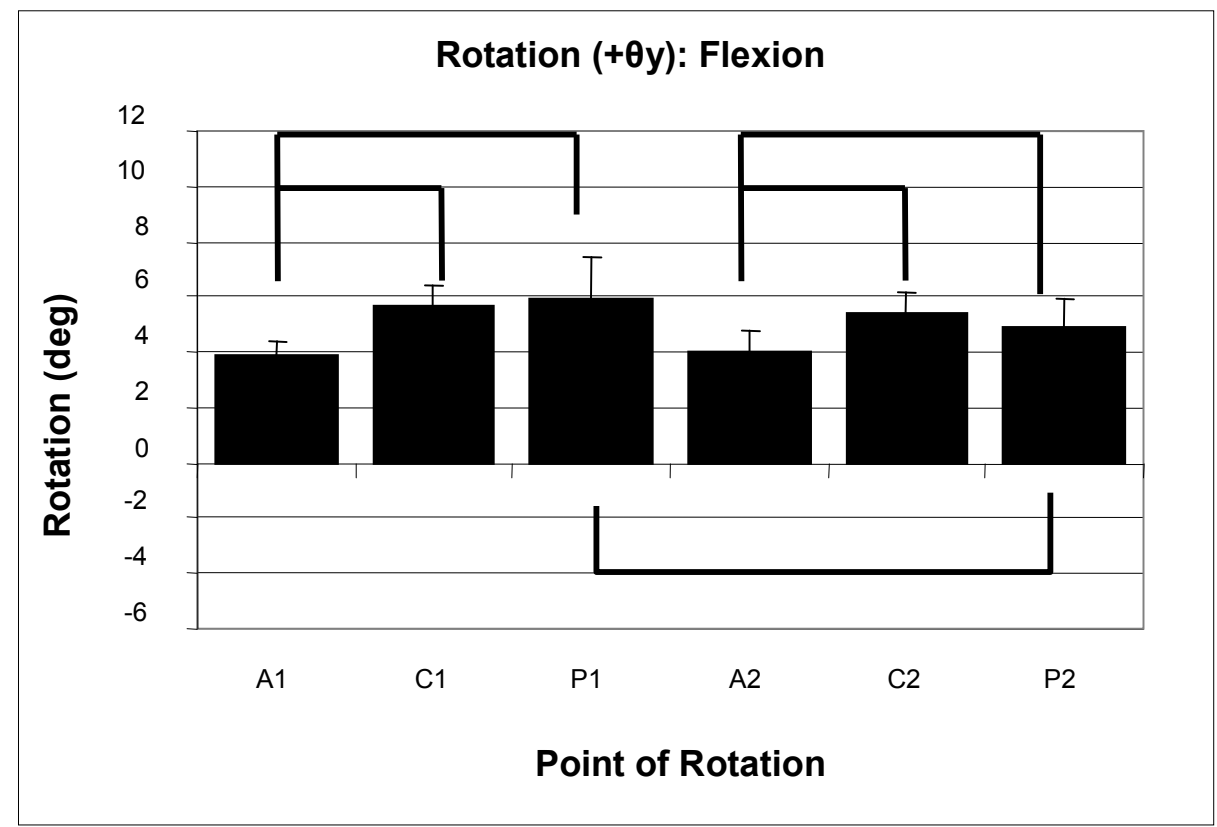

Figure 4.6 Mean Flexion Rotations.

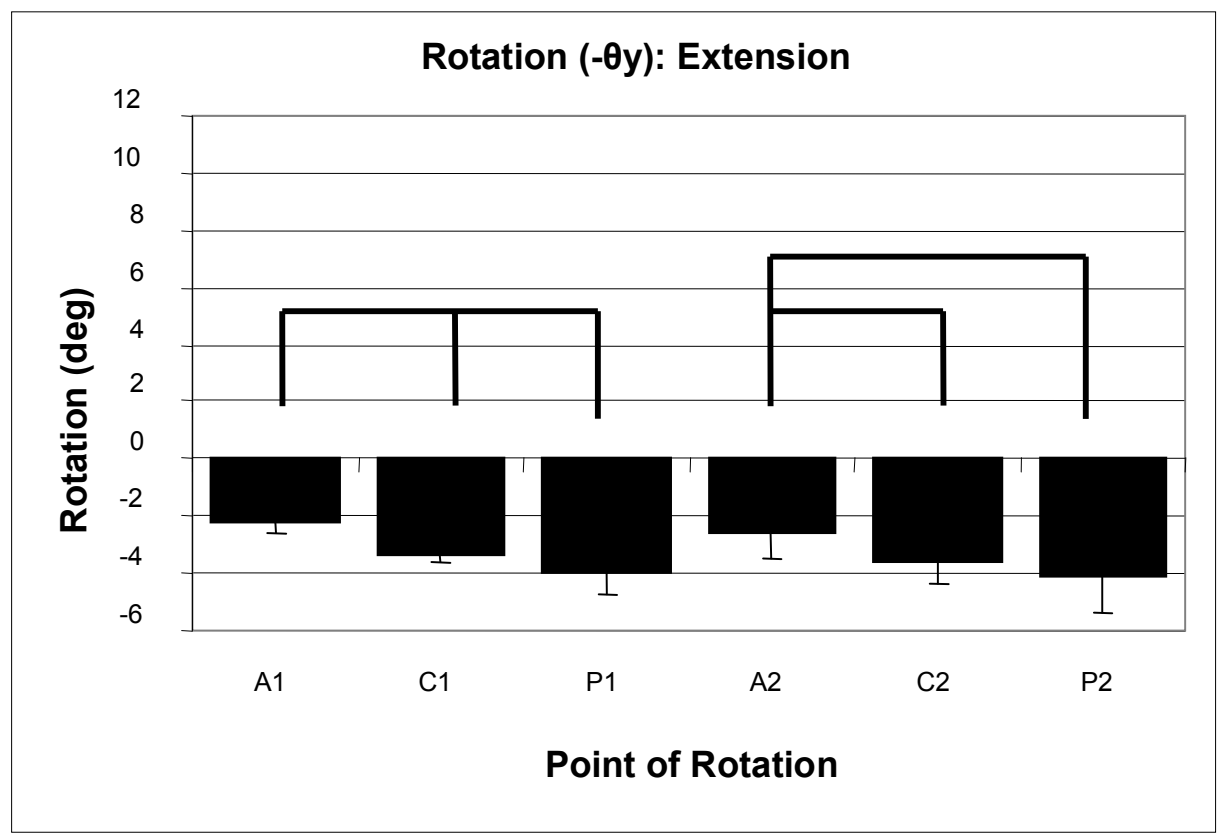

Figure 4.7 Mean Extension Rotations. 
was $5.7 \pm 0.7$ degrees and $5.5 \pm 0.7$ degrees, and rotation about the posterior points $\mathrm{P} 1$ and $\mathrm{P} 2$ was $6.0 \pm 1.5$ degrees and $4.9 \pm 0.9$ degrees, respectively. Comparisons of the inplane points along the midline showed that rotation about the central and posterior points was significantly greater than the anterior points $(\mathrm{p}<0.001)$ where $\mathrm{A} 1<\mathrm{C} 1<\mathrm{P} 1$. Interplane comparisons showed that the location of the $\mathrm{COR}$ in the cranial-caudal direction had little or no significant effect on the specimen rotation.

In extension, rotation about the anterior points $\mathrm{A} 1$ and $\mathrm{A} 2$ was $-2.2 \pm 0.5$ degrees and $-2.6 \pm 0.9$ degrees, respectively (Figure 4.7 ). Rotation about the central points $\mathrm{C} 1$ and $\mathrm{C} 2$ was $-3.3 \pm 0.3$ degrees and $-3.6 \pm 0.8$ degrees and rotation about the posterior points $\mathrm{P} 1$ and $\mathrm{P} 2$ was $-4.0 \pm 0.8$ degrees and $-4.1 \pm 1.2$ degrees, respectively. The inplane comparisons along the midline showed significant differences between all points. Rotation increased as the point of rotation moved in an anterior to posterior direction (i.e. $\mathrm{A} 1<\mathrm{C} 1<\mathrm{P} 1)$. There were no statistical differences in the inter-plane comparisons. 


\section{Chapter 5: Discussion}

Chapter five is broken into two sections. The first section discusses the relationship between the results presented in Chapter 4 and the anatomical and physiological interactions of the human lumbar MSU. The second section explains the strengths and limitations of the newly developed kinematics-based testing protocol.

\subsection{Matching Load Data to Anatomical and Physiological Interactions}

The load data presented in Section 4.1 is difficult to interpret as a data set without relating it back to what is physically happening in the spine. Basic MSU mechanics were covered in Section 2.2 and will be briefly revisited here. As a specimen is rotated in forward flexion the anterior part of the MSU is put into compression and the posterior part of the MSU is put into tension. This means that the anterior portion of the disc, principally the annulus, is being compressed and the posterior ligaments and soft tissue are being stretched in tension. The opposite is true for a backward extension motion. The axial and shear force data presented in Section 3.1 show that, depending on where the center of rotation is located, the forces and rotations are significantly different.

Figure 5.1 shows resultant force vectors calculated from the shear and axial forces. Force data for flexion and extension rotation about all six defined points of rotation are shown. This figure offers insight into why significant differences in the forces occur for different locations of the COR. Flexion force vectors are shown as solid lines and extension force vectors are dashed lines.

Tables 5.1 and 5.2 show the resultant magnitudes of each of the axial-shear force calculations as well as the angle of the resultant vector for flexion and extension rotation. The resultant force vector was calculated as:

$$
F_{R}=\sqrt{\left(F_{A}\right)^{2}+\left(F_{S}\right)^{2}}
$$

Equation 5.1

and the vector angle was calculated as:

$$
\theta=\tan ^{-1}\left(\frac{F_{A}}{F_{S}}\right)
$$

The upper-left drawing in Figure 5.1 shows flexion and extension rotations about point A1. Flexion about this point results in a large tensile force with a posterior oriented shear force. This force is a result of the tension in the disc, posterior longitudinal ligament, ligamentum flavum, interspinous ligament, and other soft tissue in the posterior part of the specimen. In extension the dominating anatomical structure is the facet joint which engages almost immediately at this point and results in a large compressive force and small anterior oriented shear force. The force vectors for the central point $\mathrm{C} 1$, shown in the upper-middle drawing, are very similar to those of point A1. 


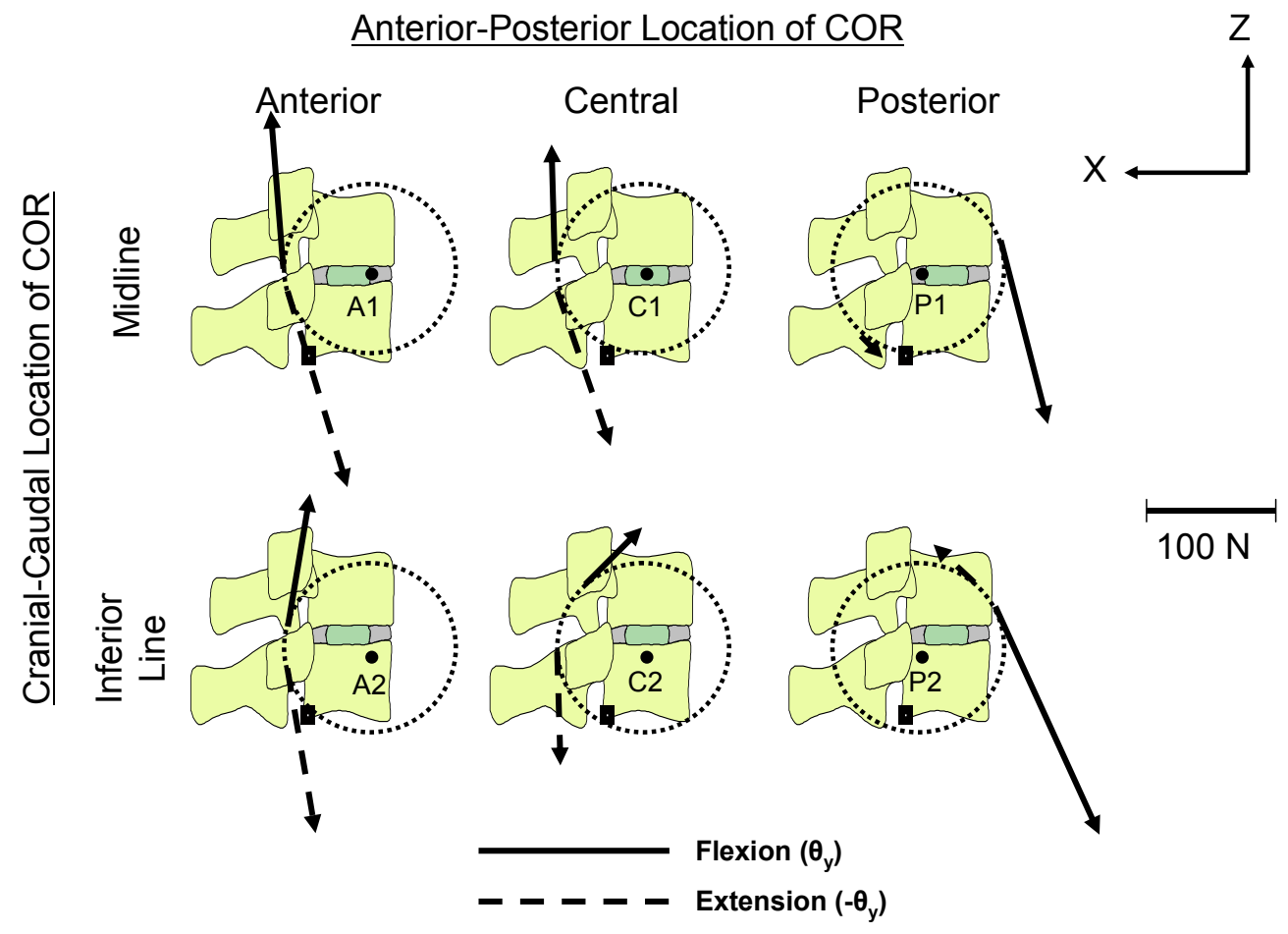

Figure 5.1 Resultant Force Vectors. 
Table 5.1 Resultant Force Vector and Vector Angle for Flexion Rotation.

\begin{tabular}{ccccc}
\hline Point & $\begin{array}{c}\text { Shear Force } \\
(\mathrm{N})\end{array}$ & $\begin{array}{c}\text { Axial Force } \\
(\mathrm{N})\end{array}$ & $\begin{array}{c}\text { Vector Angle Theta } \\
(\text { degrees })\end{array}$ & $\begin{array}{c}\text { Vector Magnitude } \\
(\mathrm{N})\end{array}$ \\
\hline A1 & 17.8 & 214.7 & 85.3 & 215.4 \\
A2 & -28.0 & 181.0 & -81.2 & 183.2 \\
C1 & 7.7 & 158.1 & 87.2 & 158.3 \\
C2 & -78.6 & 78.5 & -45.0 & 111.1 \\
P1 & -65.4 & -251.6 & 75.4 & 260.0 \\
P2 & -141.5 & -313 & 65.7 & 343.5 \\
\hline
\end{tabular}

Table 5.2 Resultant Force Vector and Vector Angle for Extension Rotation.

\begin{tabular}{ccccc}
\hline Point & Shear Force $(\mathrm{N})$ & Axial Force $(\mathrm{N})$ & $\begin{array}{c}\text { Vector Angle Theta } \\
\text { (degrees) }\end{array}$ & $\begin{array}{c}\text { Vector Magnitude } \\
(\mathrm{N})\end{array}$ \\
\hline A1 & -83.8 & -263.5 & 72.4 & 276.5 \\
A2 & -37.2 & -220.7 & 80.4 & 223.8 \\
C1 & -71.0 & -210.3 & 71.3 & 222.0 \\
C2 & -3.5 & -154.7 & 88.7 & 154.7 \\
P1 & -29.1 & -30.3 & 46.2 & 42.0 \\
P2 & 56.3 & 49.8 & 41.5 & 75.2 \\
\hline
\end{tabular}


As the COR is moved to the posterior, as seen in the upper right hand drawing of Figure 5.1, the flexion rotation is now dominated by the compression of the annulus in the anterior part of the specimen. The result is a large compressive force with a small anterior oriented shear. In extension, the facet joint does not dominate the motion, but instead the tension in the anterior annulus balances the compression of the facet; the resulting compressive force is very small with an anterior oriented shear force.

Looking at the drawings for rotation about the lower CORs, it is evident that the force magnitudes between the two planes are similar. Though the magnitudes are similar, the vector angle is different. This difference in the vector angle is a result of the different shear forces between the two planes. For all of the lower CORs, the shear force was more anterior oriented than the midline points in forward flexion and more posterior oriented in extension rotation. This difference in the shear values is not completely understood, but it is thought to be associated with facet joint and soft tissue interactions.

\subsection{Strengths and Limitations of the Kinematics-Based Testing Protocol}

Flexibility testing protocols are not capable of fully evaluating the effects of modern spine devices and instrumentation on the spine. In order to understand the effect of implanting modern spinal devices, testing protocols must be able to measure the basic kinematics and force components associated with spinal movement. The new kinematics-based testing protocol described herein had the capacity to detect significant differences in the segmental rotation and shear and axial forces for small perturbations (8-9 $\mathrm{mm}$ ) in the MSU location of the COR for both flexion and extension. This leads the author to believe that this new protocol has sufficient sensitivity to measure the effects of non-fusion devices and instrumentation, in terms of kinematics and mechanics, on the spine.

Implantable spinal devices can alter lumbar spine mechanics by changing the inherent kinematics or basic mechanical properties of the spine. For example, ProDisc implants impose a fixed COR which may be different from that of the inherent spine. These devices must then withstand and maintain fixation when subjected to altered loading conditions. The kinematics-based testing protocol is capable of evaluating the effects of such devices on the spine.

The kinematics-based testing protocol is unique in its ability to specify multiple CORs in the specimen and then measure the kinematics and mechanics responses of the MSU to a rotational input about these points individually. To the knowledge of the author there are no other testing systems being used in spine biomechanics with this capability. This puts the lab in a position to provide information about total disc replacement, nucleus replacement, and dynamic stabilization devices which is not available from any other lab. The shear force associated with physiologic and pathologic spine motion is one of the unique parameters that can be measured using this protocol. 
The protocol has been used to test the influence of a compliant nucleus replacement device (Hydraflex ${ }^{\mathrm{TM}}$, Raymedica, Minneapolis, $\mathrm{MN}$ ) on the lumbar spine. In this study it was found that an MSU implanted with a compliant nucleus replacement material was better able to accommodate physiologic flexion and extension rotations than a denucleated MSU. This is another example of a data set which can only be compiled through the use of the kinematics-based testing protocol.

One of the limitations of the protocol stems from error associated with measuring the COR from radiographic images. This error is due to the radiographic distortion inherent in fluoroscopic images.

The protocol also constrained motion to flexion-extension in the sagittal plane. In the in-vivo case, motion is rarely limited to a single plane and coupled motion is common. Slight misalignment of the potted specimens also introduced coupling in some of the specimens even though care was taken to constrain motion to only the sagittal plane. This coupling resulted in off-axis loads and moments which were monitored throughout the testing to insure that they did not exceed an acceptable level. Another limitation was the simplified loading scenario presented in this study. The responses to the CORs considered only the intact, passive spine and no efforts were made to model or assess the effects of muscle activity or external loads.

Finally, six of the specimens used in this study were L3-L4 segments of the lumbar spine and one was segment L5-S1. Data from the L5-S1 specimen differed from that of the L3-L4 specimens. The discrepancy was not statistically different but it did introduce several outlier points and tended to increase the standard deviation values. In future studies a more uniform grouping of spine segments should be used to eliminate this source of error. 


\section{Chapter 6: Conclusion and Recommendations for Future Work}

As new technologies evolve, the methods utilized to analyze their biomechanical performance must evolve. The preliminary data from this study demonstrated that utilizing a spine robot to change the location of the MSU axis of rotation had an effect on the kinematic response of the intact harvested lumbar MSU. Since the in-vivo kinematics of the lumbar MSU are not fully understood, evaluating the response at multiple locations of rotation may more effectively characterize biomechanical properties of both healthy and diseased intervertebral discs. Additional testing is being performed to further evaluate the utility of this method as well as the restorative effects of compliant implants on denucleated lumbar MSUs.

One of the more intriguing future uses of the protocol would be to evaluate the effects of implanting a TDR device in multiple A-P locations. Intra-operatively the placement of the TDR device is a subjective decision made by the surgeon. This leads to implants that are placed far posterior or anterior to their intended position. The protocol could be used to simulate these varied positions and measure the effects.

It would also be interesting to evaluate the design of TDR devices with a fixed ball-and-socket design. The protocol could be programmed to mimic different design parameters (including location of the COR) and then offer design inputs for a next generation device. Finally, a load controlled study evaluating the location of the physiologic COR in the lumbar spine would help to improve the current protocol and to provide a better understanding of the COR. 


\section{List of References}

1. Adams M, Bogduk N, Burton K, and Dolan P. The biomechanics of back pain. 1 ed. Edinburgh: Churchill Livingstone, 2002.

2. Asmussen E, Klausen K. Form and function of the erect human spine. Clin.Orthop. 1962;25:55-63.

3. Bao QB, McCullen G, Higham PA, Dumbleton Jh, and Yuan H. The artificial disc: theory, design and materials. Biomaterials 1996;17:1157-67.

4. Bonin H. In-vitro analysis of the instantaneous center of rotation in a human cervical spine model using a spine robot. Thesis, 2006. The University of Tennessee Health Science Center.

5. Butler J, Lewis R, and Shier D, eds., Hole's human anatomy \& physiology. 9 ed. Boston: McGraw Hill, 2002.

6. Chung SS, Lee CS, and Kang CS. Lumbar total disc replacement using ProDisc II: a prospective study with a 2-year minimum follow-up. J Spinal Disord Tech. 2006;19:411-5.

7. Clark CR. The cervical spine. Philadelphia: Lippincott-Raven, 1998:785.

8. Cocchiarella L, Andersson G. Guides to the evaluation of permanent impairment by the American Medical Association. 5 ed. AMA Press, 2000.

9. Crawford MJB, Doherty BJP, Dreyzin VM, and Esses SIM. Kinematic evaluation of lumbar fusion techniques. Spine 1996;21:676-84.

10. Crawford NR. Letter to the Editor. Clin Biomech 2007;861-2.

11. Crisco JJ III, Chen X, Panjabi MM, and Wolfe SW. Optimal marker placement for calculating the instantaneous center of rotation. J Biomech 1994;27:1183-7.

12. Cunningham BW, Gordon JD, Dmitriev AE, Hu N, and McAfee PC.

Biomechanical evaluation of total disc replacement arthroplasty: an in-vitro human cadaveric model. Spine 2003;28:S110-S117.

13. Dabnichki P, Lauder M, Aritan S, and Tsirakos D. Accuracy evaluation of an online kinematic system via dynamic tests. J Med Eng Technol 1997;21:53-66.

14. Deguchi M, Cheng BC, Sato K, Matsuyama Y, and Zdeblick TA. Biomechanical evaluation of translaminar facet joint fixation. A comparative study of poly-Llactide pins, screws, and pedicle fixation. Spine 1998;23:1307-12. 
15. DiAngelo DJ, Foley KT. An improved biomechanical testing protocol for evaluating multilevel cervical instrumentation in a human cadaveric corpectomy model. In: Melkerson MN, Kirkpatrick JS, Griffith SL, eds. Spinal implants: are we evaluating them appropriately? STP1431. West Conshohocken, PA: ASTM International, 2003:155.

16. DiAngelo DJ, Roberston JT, Metcalf NH, McVay BJ, and Davis RC.

Biomechanical testing of an artificial cervical joint and an anterior cervical plate. $\mathrm{J}$ Spinal Disord Tech 2003;16:314-23.

17. DiAngelo DJ, Schwab J, and Foley KT. Motion compensation associated with single-level cervical fusion: where does the lost motion go? Spine 2006;31:2439-48.

18. DiAngelo DJ, Scifert J, Kitchel S, Cornwall G, and McVay BJ. Bioabsorbable anterior lumbar plate fixation in conjunction with cage-assisted anterior interbody fusion. J.Neurosurg. 2002;4:447-55.

19. DiAngelo DJ, Vossel KA, and Jansen TH. A multi-body optical measurement system for the study of human joint motion. BED 1998;39:195-6.

20. Dickey JP, Gillespie KA. Representation of passive spinal element contributions to in-vitro flexion-extension using a polynomial model: illustration using the porcine lumbar spine. J Biomech 2003;36:883-8.

21. Dooris A, Goel V, Grosland NM, Gilbertson L, and Wilder DG. Load-sharing between anterior and posterior elements in a lumbar motion segment implanted with an artificial disc. Spine 2001;26:E122-E129.

22. Goel VK, Clark CR, McGowan D, and Goyal S. An in-vitro study of the kinematics of the normal, injured and stabilized cervical spine. J Biomech 1984;17:363-76.

23. Goel VK, Goyal S, Clark C, Nishiyama K, and Nye T. Kinematics of the whole lumbar spine. Effect of discectomy. Spine 1985;10:543-54.

24. Goel VK, Panjabi MM, Patwardhan AG, Dooris AP, and Serhan H. Test protocols for evaluation of spinal implants. J of Bone and Joint Surgery-American Volume 2006;88A:103-9.

25. Goel VK, Wilder DG, Pope MH, and Edwards WT. Biomechanical testing of the spine. Load-controlled versus displacement-controlled analysis. Spine 1995;20:2354-7.

26. Grassmann S, Oxland TR, Gerich U, and Nolte LP. Constrained testing conditions affect the axial rotation response of lumbar functional spinal units. Spine 1998;23:1155-62. 
27. Gray H. Anatomy of the human body. 20 ed. Philadelphia: Lea \& Febiger, 1918.

28. Hasegawa K, Kitahara K, Hara T, Takano K, Shimoda H, and Homma T. Evaluation of lumbar segmental instability in degenerative diseases by using a new intraoperative measurement system. J Neurosurg Spine 2008;8:255-62.

29. Holzreiter S. Calculation of the instantaneous centre of rotation for a rigid body. $\mathrm{J}$ Biomech 1991;24:643-7.

30. Humzah MD, Soames RW. Human intervertebral disc: structure and function. Anat Rec 1988;220:337-56.

31. Ianuzzi A, Zambrano I, Tataria J et al. Biomechanical evaluation of surgical constructs for stabilization of cervical teardrop fractures. Spine J. 2006;6:514-23.

32. Jeon S, Choi W, and Lee S. Anterior revision of a dislocated ProDisc prosthesis at the L4-L5 level. J Spinal Disord Tech 2008;21:448-50.

33. Johnson WM, Rapoff AJ. Three degree of freedom biomechanical testing system. Proceedings of the American Society of Mechanical Engineers, November, New York, NY, 2001.

34. Jones GA, Kayanja M, Milks R, and Lieberman I. Biomechanical characteristics of hybrid hook-screw constructs in short-segment thoracic fixation. Spine 2008;33:173-7.

35. Kanayama M, Cunningham BW, Weis JC, Parker LM, Kaneda K, and McAfee PC. The effects of rigid spinal instrumentation and solid bony fusion on spinal kinematics. A posterolateral spinal arthrodesis model. Spine 1998;23:767-73.

36. Kelly BP. A Multiaxis programmable spine robot for the study of multibody spinal biomechanics using real-time hybrid force and displacement control strategies.

Dissertation, 2005. The University of Tennessee Health Science Center, Memphis.

37. Kunz DN, McCabe RP, Zdeblick TA, and Vanderby R J. A multi-degree of freedom system for biomechanical testing. J Biomech Eng 1994;116:371-3.

38. Manchikanti L, Manchikanti K, Cash K, Singh V, and Giordano J. Age-Related prevalence of facet-joint involvement in chronic neck and low back pain. Pain Physician 2008;11:67-75.

39. Martin MD, Boxell CM, and Malone DG. Pathophysiology of lumbar disc degeneration: a review of the literature. Neurosurgical Focus 2002;13:1-6. 
40. Mathew P, Blackman M, and Redla S. Bilateral pedicle fractures following anterior dislocation of the polyethylene inlay of a ProDisc artificial disc replacement: a case report of an unusual complication. Spine 2005;30:E311-E314.

41. Ogston NG, King GJ, Gertzbein SD, Tile M, Kapasouri A, and Rubenstein JD. Centrode patterns in the lumbar spine: baseline studies in normal subjects. Spine 1986;11:591-5.

42. Panjabi M. Clinical spinal instability and low back pain. J Electromyogr Kinesiol 2003;13:371-9.

43. Panjabi M. Hybrid Multidirectional Test Method to Evaluate Spinal Adjacent-Level Effects. Clin Biomech 2007;257-65.

44. Panjabi MM. Biomechanical evaluation of spinal fixation devices: I. A conceptual framework. Spine 1988;13:1129-34.

45. Panjabi MM. The stabilizing system of the spine. Part II. Neutral zone and instability hypothesis. J Spinal Disord 1992;5:390-6.

46. Panjabi MM, Lydon C, Vasavada A, Grob D, Crisco JJ I, and Dvorak J. On the understanding of clinical instability. Spine 1994;19:2642-50.

47. Panjabi MM, Yamamoto I, Oxland T, Crisco JJ, and Freedman D. Biomechanical stability of five pedicle screw fixation systems in a human lumbar spine instability model. Clin Biomech 1991;6:197-205.

48. Panjabi M, Henderson G, Abjornson C, and Yue J. Multidirectional testing of oneand two-level ProDisc-L versus simulated fusions. Spine 2007;32:1311-9.

49. Panjabi MM. Hybrid multidirectional test method to evaluate spinal adjacent-level effects. Clin Biomech 2007;22:257-65.

50. Patwardhan AG, Havey RM, Meade KP, Lee B, and Dunlap B. A follower load increases the load-carrying capacity of the lumbar spine in compression. Spine 1999;24:1003-9.

51. Pearcy MJ, Bodguk N. Instantaneous axes of rotation of the lumbar intervertebral joints. Spine 1988;13:1033-41.

52. Pearcy MJ, Tibrewal S. Lumbar intervertebral disc and ligament deformation measured in-vivo. Clin Orthop 1984;191:281-6.

53. Porterfield, DeRosa. Mechanical Neck Pain. Boston. W.B.Saunders, 1995. 
54. Roberts S. Disc Morphology in health and disease. Biochemical Society Transactions 2002;30:864-9.

55. Rousseau M, Bradford DS, Bertagnoli R, Hu S, and Lotz JC. Disc Arthroplasty design influences intervertebral kinematics and facet forces. The Spine Journal 2006;258-66.

56. Sakamaki T, Katoh S, and Sairyo K. Normal and spondylolytic pediatric spine movements with reference to instantaneous axis of rotation. Spine 2002;27:141-5.

57. Schneider G, Pearcy MJ, and Bogduk N. Abnormal Motion in Spondylolytic Spondylolisthesis. Spine 2005;30:1159-64.

58. Schwab JS. Motion compensation associated with cervical fusion: A multi-level invitro study. Thesis, 2003. University of Tennessee Health Science Center, Memphis.

59. Seligman JV, Gertzbein SD, Tile M, and Kapasouri A. Computer analysis of spinal segment motion in degenerative disc disease with and without axial loading (Volvo award in basic science). Spine 1984;9:566-73.

60. Sobajima S, Kim JS, Gilbertson LG, and Kang J. Gene therapy for degenerative disc disease. Gene Therapy 2004;11:390-401.

61. Torzilli PA, Grigiene R, Huang C et al. A mechanical explant test system for articular cartilage design, evaluation and preliminary results. 1993:438-441.

62. White AA, Panjabi M. Clinical biomechanics of the spine. 2 ed. Philadelphia: J.B. Lippincott Co., 1990.

63. Wilke HJ, Claes L, Schmitt H, and Wolf S. A universal spine tester for in-vitro experiments with muscle force simulation. Eur Spine J 1994;3:91-7.

64. Yang Y, Nie L, Cheng L, and Hou Y. Clinical and radiographic reports following cervical arthroplasty: a 24 month follow-up. Int Orthop 2008;E-publication.

65. Yoshioka T, Tsuji H, Hirano N, and Sainoh S. Motion characteristics of the normal lumbar spine in young adults: instantaneous axis of rotation and vertebral center motion analyses. J Spine Disord 1990;3:103-13.

66. Zindrick MR, Tzermiadianos MN, Voronov LI, Lorenz M, and Hadjipavlou A. An evidence-based medicine approach in determining factors that may affect outcome in lumbar total disc replacement. Spine 2008;33:1262-9. 


\section{Appendix A: Additional Specimen Grids}

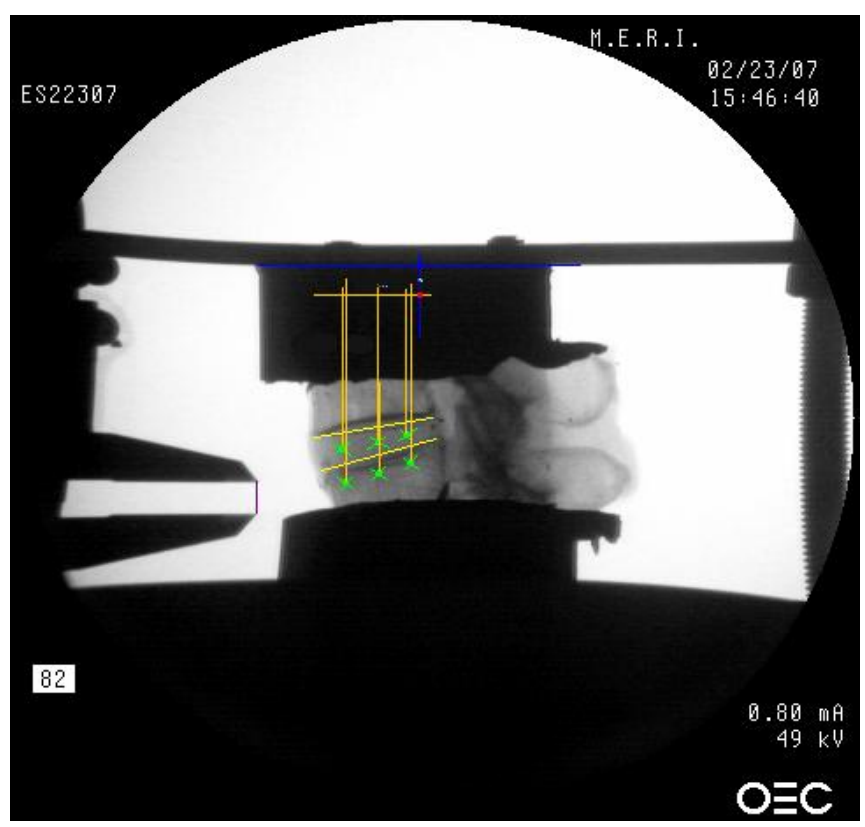

Figure A.1 Grid for Specimen 0105416 L3-L4.

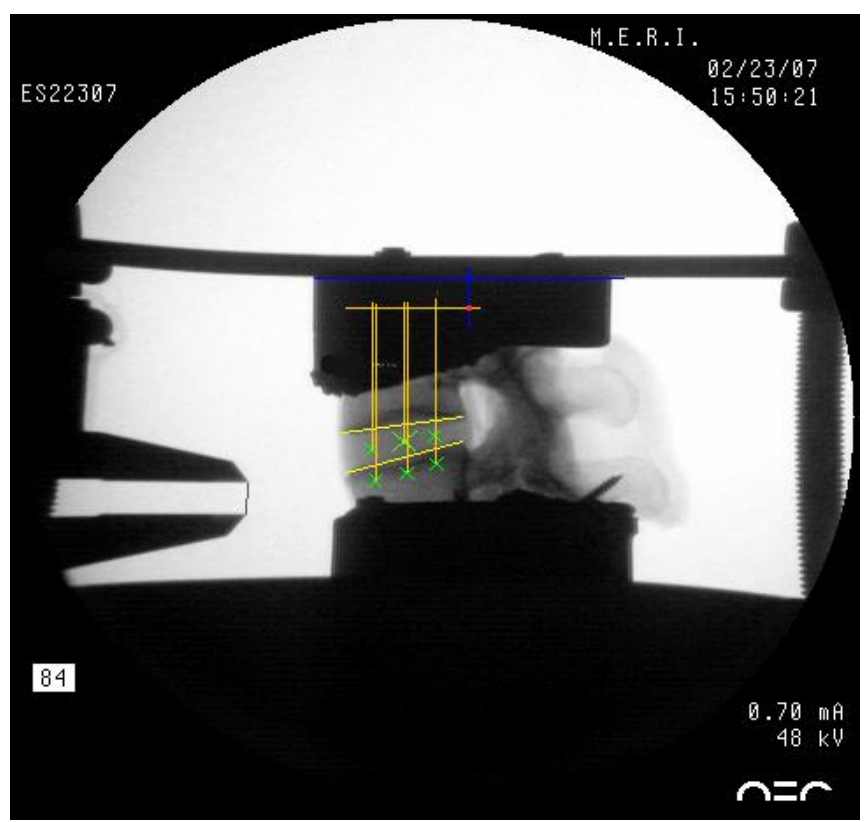

Figure A.2 Grid for Specimen ADS0612051 L3-L4. 


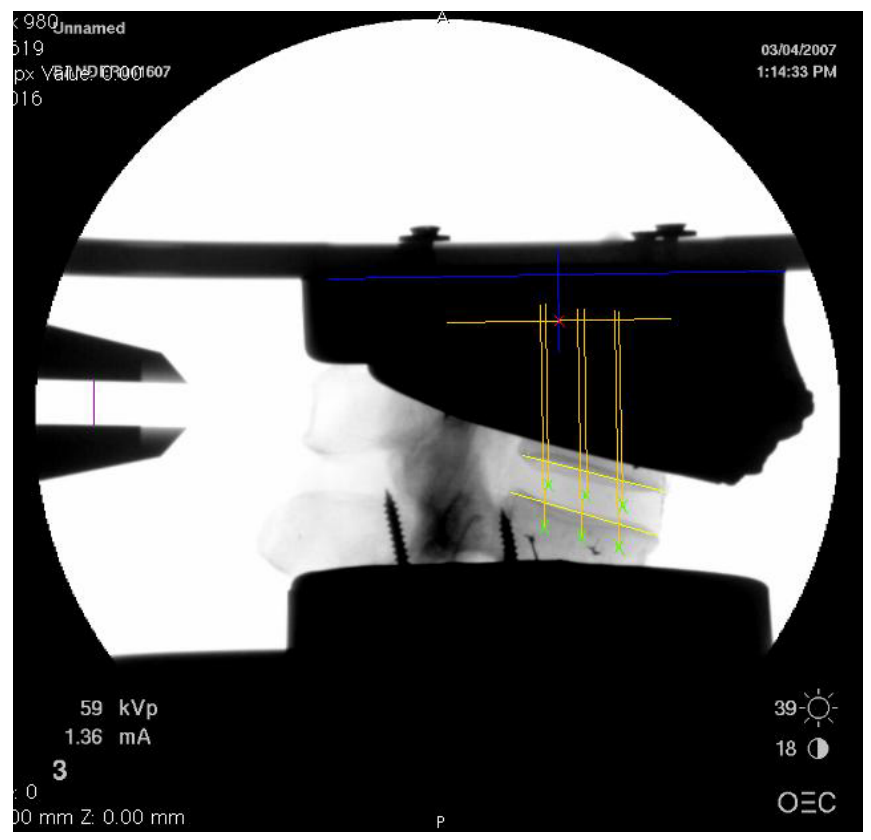

Figure A.3 Grid for Specimen DRT050786 L3-L4.

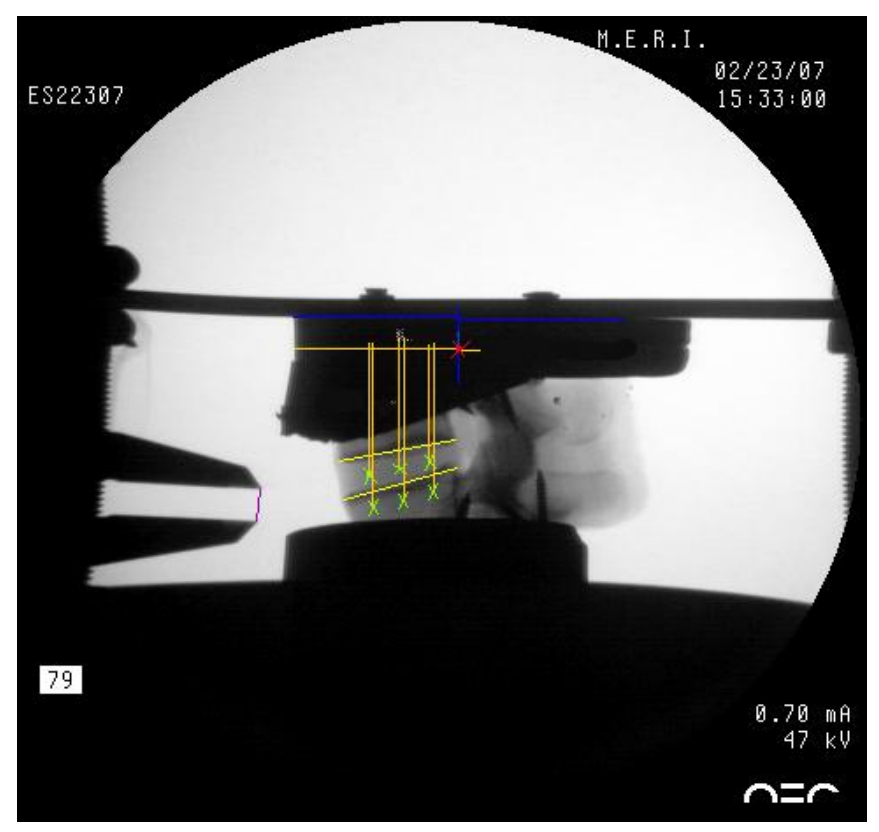

Figure A.4 Grid for Specimen DRT060069 L3-L4. 


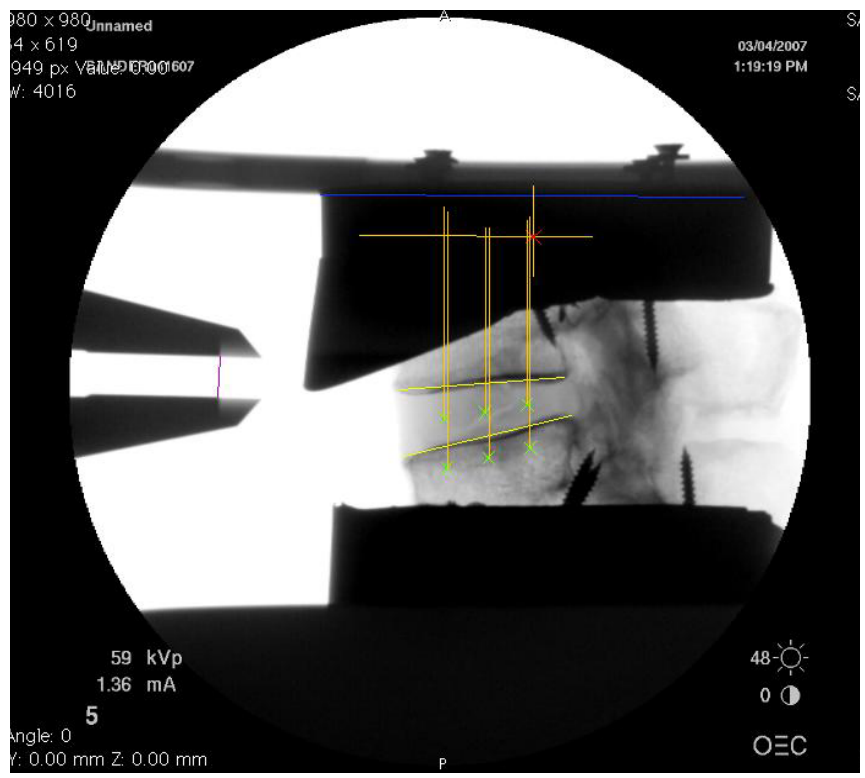

Figure A.5 Grid for Specimen UF01A030 L3-L4.

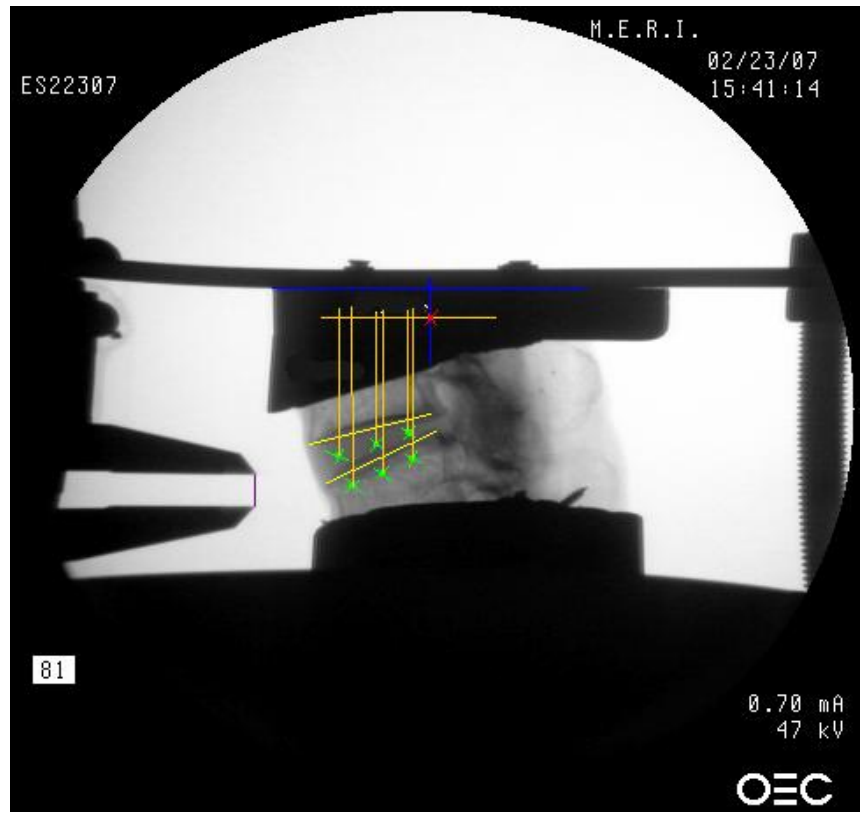

Figure A.6 Grid for Specimen UJ00D35 L3-L4. 


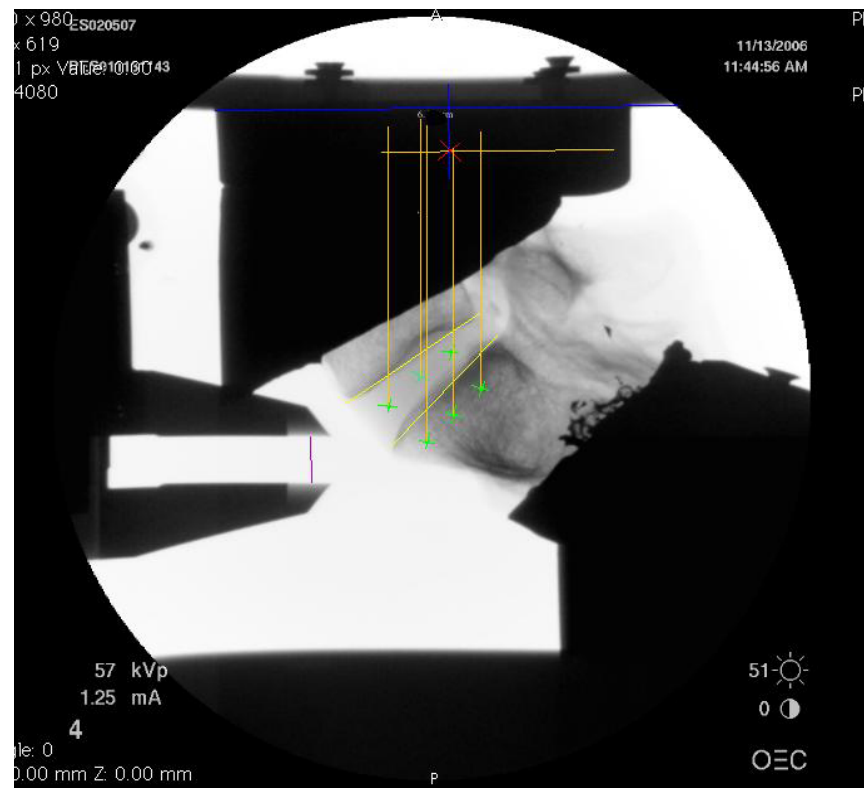

Figure A.7 Grid for Specimen ADS0612051 L5-S1. 


\section{Appendix B: Spine Robot Control Programs}

\section{B.1 Fixed_Axis1}

;ABSTRACT: This program is intended to be able to apply a known and fixed axis of ;rotation wherever you want within a specimen. AOR values were determined based on ;flouro scans, and a grid was generated based on the assumption that the anterior edge of ;the top pot will be flush with the connector plate during testing.

;

; INPUT PARMS:

; OUTPUT PARMS:

; SIDE EFFECTS:

; DATA STRUCT:

; MISC: For use with Single Level specimens.

;

;* Copyright (c) 2004, Henry Bonin and Brian P. Kelly

;University of Tennessee Health Science Center, Dept. of Biomedical Engineering

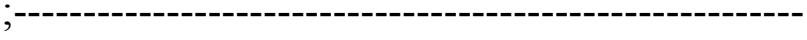

GLOBAL REAL pot.wt

DISABLE SCALE.ACCEL

ACCEL (6) 80, 80

SPEED 100 MONITOR

SPEED 20 ALWAYS

ai.version $=1302 \quad$;must be included in order to use the force buffer

pot.wt $=0$

SELECT FORCE $=2 \quad$;select and initialize base load cell paramenters

FORCE.MODE (21)

FORCE.MODE (10) 4.45

FORCE.MODE (11) 0.0254

FORCE.MODE (12) 5

FORCE.MODE (13) 3

FORCE.MODE (14) 6

FORCE.OFFSET (0)

SELECT FORCE $=1$

;select and initialize upper load cell parameters

FORCE.MODE (21) 
; FORCE.FRAME (1) TRANS(,,-51.549) ;this accounts for the offset of the specimmen ;from the load cell

FORCE.MODE (10) 4.45

FORCE.MODE (11) 0.0254

FORCE.MODE (12) 5 ; this value will influence output, 5 is best

FORCE.MODE (13) 3

FORCE.MODE (14) 6

FORCE.OFFSET (0)

; FORCE.MODE (1)^H11, 20, ^H511, 1 ;guarded for out of plane forces and ;moments

; FORCE.MODE (1) ${ }^{\wedge} \mathrm{H} 21,75,{ }^{\wedge} \mathrm{H} 121,0.5$;guarded for in plane moments

FORCE.MODE (1) ${ }^{\wedge} \mathrm{H} 430,75, \wedge \mathrm{H} 131,1.25$;guarded for $3 \mathrm{D}$ resultant foces and ; X-z plane moments

TYPE /C5

1 PROMPT "Enter a value for the weight of upper potted fixture(Newtons) ", pot.wt IF (pot.wt $<0$ ) THEN

TYPE "You must enter a positive value!" GOTO 1

END

IF (pot.wt > 100) THEN

PROMPT "That is a large value, are you sure? (Y/N)", \$ans

IF ( \$ans <> "Y") AND (\$ans <> "N") OR (\$ans == "N") THEN GOTO 1

END

END

CALL spine_mount23()

FORCE.MODE (-1)

;disable any guarded moves in Spine.mount FORCE.MODE (-1)

FORCE.MODE (1)^H430, 75, ^H530, 1.25 ;guarded for forces and moments TYPE /C50,/U42

3 SELECT FORCE $=1$

SET a.point $=\operatorname{TRANS}(0,0,0) ;$ Set null tool transformation

TOOL a.point

FORCE.FRAME (1) TRANS $(0,0,0)$;Set null load cell reference frame transformation

; FORCE.FRAME (-1), reframe

; DECOMPOSE loadframe[] = reframe

; TYPE "load cell reference frame x value is ", loadframe[0] 
; $\quad$ TYPE "load cell reference frame $\mathrm{z}$ value is ", loadframe[2]

; $\quad$ PROMPT "Did you see this ", \$ans10

HERE start.point. 1

DECOMPOSE $\mathrm{sp}[]=$ start.point. 1

$\mathrm{xstart}=\mathrm{sp}[0]$

zstart $=\mathrm{sp}[2]$

; $\quad$ PROMPT "Do you need to change the tool transformation?(y/n): ", \$ans

; $\quad$ IF (\$ans $<$ "y") AND (\$ans $<>$ "n") GOTO 4

; $\quad$ IF $\$$ ans $==$ "y" GOTO 5

; $\quad$ IF $\$$ ans $==$ "n" GOTO 6

TYPE /C50, /U42

TYPE /C5

TYPE /X20, "****TOOL TRANSFORMATION INFORMATION****"

TYPE /C2

PROMPT "What is the value of your tool transformation in the WCS x direction?: ", xvalue

TYPE /C1

1000 PROMPT "What is the value of your tool transformation in the WCS z direction?

(CAUTION! : this must be negative!): ", zvalue

IF (zvalue > 0) THEN

TYPE "The $\mathrm{z}$ value must be negative."

GOTO 1000

END

SET this.point $=$ TRANS(xvalue,,-zvalue $) \quad ;(-)$ zvalue to cancel the $(-)$ entered in prompt - for our system this must be positive

TOOL this.point

FORCE.FRAME (1) TRANS(-xvalue,,zvalue-51.549)

TYPE /C50, /U42

TYPE /C5

PROMPT "What is the value of your 3D resultant force trip condition (Newtons)? ", for.trip

TYPE /C3

PROMPT "What is the value of your sagital plane moment trip condition (Nm)? ", mom.trip

; Code to zero in $\mathrm{x}$ direction 
2 SELECT FORCE $=1$

TYPE " "

TYPE " Current load cell readings are:"

TYPE " Fx Fy Fz Mx My Mz"

FORCE.READ (0) f[]

TYPE /F8.2, f[0], f[1], f[2], f[3], f[4], f[5]

TYPE /C1

4 PROMPT "Would you like to zero in the $\mathrm{x}$ direction? (y/n)", \$ans

IF (\$ans <> "y") AND (\$ans <> "n") GOTO 4

IF \$ans == "y" GOTO 5

IF \$ans == "n" GOTO 6

5 FORCE.READ (0) f[]

IF $(\mathrm{f}[0]<0)$ THEN

FORCE.MODE (1)^H10, 0

ACCEL (2) 100, 100

DRIVE $1,-5,5$

BREAK

FORCE.MODE (-1)

ELSE

FORCE.MODE $(1)^{\wedge} \mathrm{H} 210,0$

ACCEL (2) 100, 100

DRIVE 1, 5, 5

BREAK

FORCE.MODE (-1)

END

WAIT.EVENT , 0.2

IF LATCHED(1) THEN

TYPE /C1

TYPE " GUARDED TRIGGER! Forces zeroed in x direction:"

TYPE " Fx Fy $\mathrm{Fz}$ Mx $\mathrm{My}$ Mz"

FORCE.READ (1) f[]

TYPE /F8.2, f[0], f[1], f[2], f[3], f[4], f[5]

TYPE " "

END

; $\quad$ FORCE.MODE $(-1)^{\wedge} \mathrm{H} 10,0$

WAIT.EVENT, 0.5

GOTO 2

TYPE " " 
6 TYPE /C50,/U42

TYPE /C5

TYPE /X20, "****TEST SELECTION****"

TYPE /C2

TYPE " What test would you like to perform?"

10 PROMPT " For Flexion, hit f; for Extension, hit e; or to Sweep from Ext to Flex, hit s.", \$test.ans

IF (\$test.ans $<>$ "f") AND (\$test.ans $<>$ "e") AND (\$test.ans $<>$ "s") GOTO 10

IF $\$$ test.ans $==$ "f" GOTO 20

IF $\$$ test.ans $==$ "e" GOTO 30

IF \$test.ans == "s" GOTO 40

; ****************************************************************************

;FLEXION MOVE

20 FLIP

CPON

TYPE /C5

22 PROMPT "How many degrees in FLEXION would you like to push your specimen?", flexdeg

IF (flexdeg > 10) THEN

TYPE "Wow: ", flexdeg, "degrees in flexion."

PROMPT "Are you sure?(y/n):", \$ans

IF \$ans == "n" GOTO 22

END

IF (flexdeg < 0) THEN

TYPE "Wow: ", flexdeg, "degrees in flexion."

PROMPT "Are you sure?(y/n):", \$ans

IF \$ans $==$ "n" GOTO 22

END

; $\quad$ MOVES TRANS(,,zvalue,180,180,0)

; establish flip quadrant

MOVE TRANS(sp[0]+xvalue,,sp[2]+zvalue,180,179.9,0)

;initial move to get in flip quad

BREAK

CALL spine_preload23() 
;should I incorporate the change in position due to the preload?

HERE start.point.2

DECOMPOSE sp2[] = start.point. 2

$\mathrm{xstart}=\mathrm{sp} 2[0]$

zstart $=\mathrm{sp} 2[2]$

; MOVE TRANS(sp[0]+xvalue,,sp[2]+zvalue,180,179.9,0) ;initial move to get in flip quad

; BREAK

FORCE.MODE (-1) ; ;isable any guarded moves within

spine.preload

FORCE.MODE (-1)

FORCE.MODE (1) ${ }^{\wedge} \mathrm{H} 430$, for.trip, ${ }^{\wedge} \mathrm{H} 530$, mom.trip $\quad$;guarded for 3D resultant foces and $\mathrm{x}-\mathrm{z}$ plane moments

SELECT FORCE $=2$

CALL fs.buf.config(-1, $-1,60,50$, stt)

IF stt $<0$ GOTO 100

$\mathrm{i}=1$

radius $=\operatorname{SQRT}(($ xvalue*xvalue $)+($ zvalue*zvalue $))$

beta $=$ ATAN2(xvalue,ABS(zvalue)

WAIT.EVENT , 1

SELECT FORCE $=2$

CALL fs.buf.enable()

SPEED 20

TIMER $(1)=0$

SELECT FORCE $=1$

MOVES TRANS(sp2[0],,sp2[2],180,180-flexdeg,0) ;move flexdeg degrees in flexion

WHILE STATE(2) $==1$ DO

HERE loc.start1

DECOMPOSE $\mathrm{x} 1[]=$ loc.start 1

$\operatorname{pos}[2, \mathrm{i}]=(180-\mathrm{x} 1[4])$

$\operatorname{pos}[0, \mathrm{i}]=\mathrm{x} 1[0]$

$\operatorname{pos}[1, \mathrm{i}]=\mathrm{x} 1[2]$

$; \quad \operatorname{pos}[3, \mathrm{i}]=-(\operatorname{radius} *(\operatorname{SIN}($ beta-pos$[2, \mathrm{i}])))$ 
$; \quad \operatorname{pos}[4, \mathrm{i}]=(\operatorname{ABS}($ zvalue $))+($ zvalue $+($ radius $*(\operatorname{COS}($ beta-pos $[2, \mathrm{i}]))))$

$\operatorname{pos}[3, \mathrm{i}]=\mathrm{x} 1[5]$

FORCE.READ (0) f[]

$\mathrm{f}[4, \mathrm{i}]=\mathrm{f}[4]$

shearerr[i] $=\left(-1 \mathrm{E}-05^{*} \operatorname{pos}[2, \mathrm{i}] * \mathrm{SQR}(\operatorname{pos}[2, \mathrm{i}])-4 \mathrm{E}-\right.$

$05 * \operatorname{SQR}(\operatorname{pos}[2, \mathrm{i}])+0.1746 * \operatorname{pos}[2, \mathrm{i}])$

sinpitch $=\operatorname{SIN}(\operatorname{pos}[2, \mathrm{i}])$

$\operatorname{cospitch}=\operatorname{COS}(\operatorname{pos}[2, \mathrm{i}])$

term1 $[\mathrm{i}]=$ shearerr $[\mathrm{i}]^{*}(1) *\left(0.02652+(\mathrm{ABS}(\mathrm{zvalue}))^{*} 1 \mathrm{E}-03\right)$

term2[i] $=$ pot. $w t^{*}$ sinpitch $*(\mathrm{ABS}($ zvalue $) * 1 \mathrm{E}-03)$

term3 $[\mathrm{i}]=$ pot.wt* ${ }^{*}$ ospitch*xvalue*1E-03

$\mathrm{fs}[0, \mathrm{i}]=\mathrm{f}[0]-\operatorname{shearerr}[\mathrm{i}] *(1)-\left(\right.$ pot.wt)${ }^{*} \operatorname{sinpitch}^{*}(1) \quad ; *(1)$ refers to the cosax

$\mathrm{fs}[1, \mathrm{i}]=\mathrm{f}[1]$

$\mathrm{fs}[2, \mathrm{i}]=\mathrm{f}[2]-(-1.6 \mathrm{E}-03 * \mathrm{SQR}(\operatorname{pos}[2, \mathrm{i}])+3.2 \mathrm{E}-03 * \operatorname{pos}[2, \mathrm{i}])-($ pot.wt) $*$ cospitch

$\mathrm{fs}[3, \mathrm{i}]=\mathrm{f}[3]$

$\mathrm{fs}[4, \mathrm{i}]=\mathrm{f}[4]$-shearerr[i] $*(1) *\left(0.02652+(\mathrm{ABS}(\mathrm{zvalue}))^{*} 1 \mathrm{E}-03\right)-$

pot.wt*sinpitch*(ABS(zvalue)*1E-03)+pot.wt*cospitch*xvalue*1E-03

$\mathrm{fs}[5, \mathrm{i}]=\mathrm{f}[5]$

IF LATCHED(1) GOTO 25

$\operatorname{tm} 1[\mathrm{i}]=\operatorname{TIMER}(1)$

$\mathrm{i}=\mathrm{i}+1$

WAIT.EVENT , 5E-03

END

flex.total $=\mathrm{i}$

TYPE "Final outbound (flexed) $i=$ ", i

; $\quad$ FOR $\mathrm{i}=1$ TO flex.total-1

; TYPE /F9.5, pos[2,i], shearerr[i], term1[i], term2[i], term3[i], f[4,i], fs[4,i]

; END

25 HERE loc.trip

DECOMPOSE $\mathrm{tp}[]=$ loc.trip

$\operatorname{pos}[0]=\operatorname{tp}[0]$

$\operatorname{pos}[1]=\operatorname{tp}[2]$

$\operatorname{pos}[2]=(180-\operatorname{tp}[4])$

$\operatorname{pos}[3]=\operatorname{tp}[5]$

shearerr $=(-1 \mathrm{E}-05 * \operatorname{pos}[2] * \mathrm{SQR}(\operatorname{pos}[2])-4 \mathrm{E}-05 * \mathrm{SQR}(\operatorname{pos}[2])+0.1746 * \operatorname{pos}[2])$

sinpitch $=\operatorname{SIN}(\operatorname{pos}[2])$

cospitch $=\operatorname{COS}(\operatorname{pos}[2])$

FORCE.READ (0) f[] 


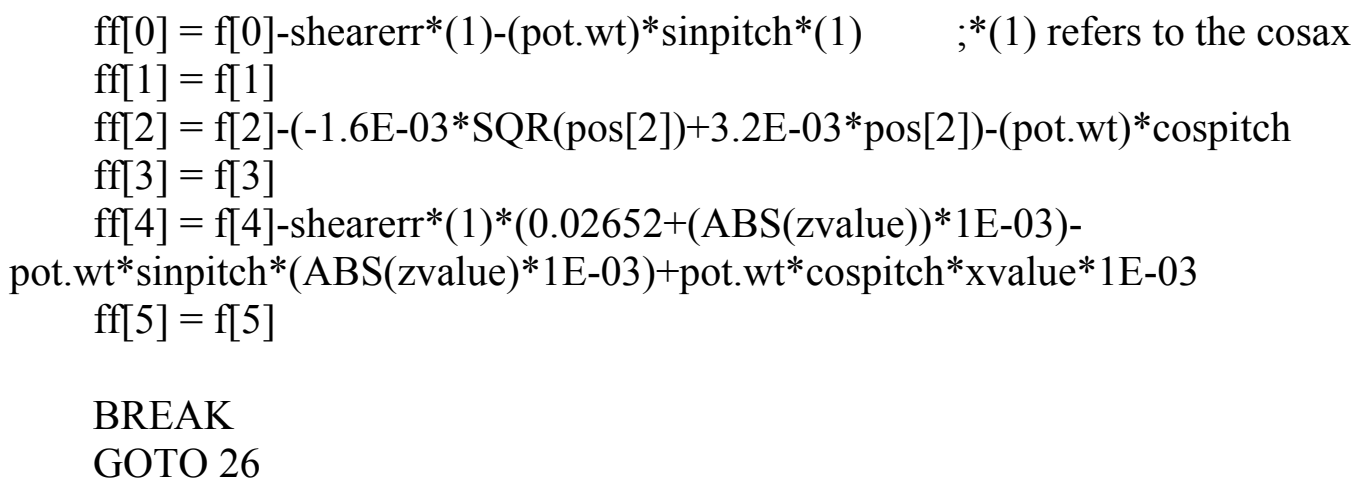




\section{END}

; $\quad$ MOVES TRANS(,,zvalue, $0,180,180)$

MOVE TRANS(sp[0]+xvalue,,sp[2]+zvalue, $0,179.9,180)$

BREAK

CALL spine_preload23()

;should I incorporate the change in position due to the preload?

HERE start.point.2

DECOMPOSE sp2[] = start.point. 2

xstart $=\mathrm{sp} 2[0]$

$\mathrm{zstart}=\mathrm{sp} 2[2]$

FORCE.MODE (-1)

FORCE.MODE (-1)

FORCE.MODE (1) ${ }^{\wedge} \mathrm{H} 430$, for.trip, ${ }^{\wedge} \mathrm{H} 530$, mom.trip $\quad$;guarded for 3D resultant foces and $\mathrm{x}-\mathrm{z}$ plane moments

SELECT FORCE $=2$

CALL fs.buf.config(-1, $-1,60,50$, stt $)$

IF $\mathrm{stt}<0$ GOTO 100

$\mathrm{i}=1$

radius $=\operatorname{SQRT}(($ xvalue $*$ xvalue $)+($ zvalue*zvalue $))$

beta $=$ ATAN2(xvalue,ABS(zvalue)

WAIT.EVENT , 1

SELECT FORCE $=2$

CALL fs.buf.enable()

SPEED 20

SELECT FORCE $=1$

$\operatorname{TIMER}(1)=0$

MOVES TRANS(sp2[0],,sp2[2],0,180-extdeg,180) ;move extdeg degrees in extension

WHILE STATE(2) $==1$ DO

HERE loc.start2

DECOMPOSE x2[] = loc.start2

$$
\begin{aligned}
& \operatorname{pos}[2, \mathrm{i}]=-(180-\mathrm{x} 2[4]) \\
& \operatorname{pos}[0, \mathrm{i}]=\mathrm{x} 2[0] \\
& \operatorname{pos}[1, \mathrm{i}]=\mathrm{x} 2[2]
\end{aligned}
$$




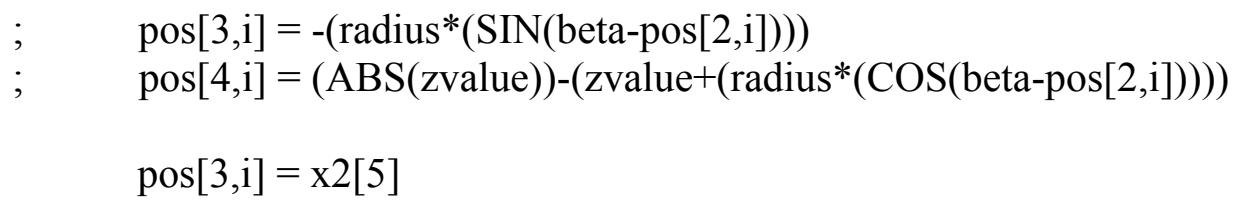

FORCE.READ (0) f[]

$\mathrm{f}[4, \mathrm{i}]=\mathrm{f}[4]$

; TYPE /F9.5, f[4], f[4,i]

shearerr $=\left(-1 \mathrm{E}-05^{*} \operatorname{pos}[2, \mathrm{i}] * \mathrm{SQR}(\operatorname{pos}[2, \mathrm{i}])-4 \mathrm{E}-\right.$

$05 * \operatorname{SQR}(\operatorname{pos}[2, \mathrm{i}])+0.1746 * \operatorname{pos}[2, \mathrm{i}])$

sinpitch $=\operatorname{SIN}(\operatorname{pos}[2, \mathrm{i}])$

cospitch $=\operatorname{COS}(\operatorname{pos}[2, \mathrm{i}])$

term $1[\mathrm{i}]=$ shearerr* $(1) *(0.02652+(\mathrm{ABS}(\mathrm{zvalue})) * 1 \mathrm{E}-03)$

term2[i] $=$ pot.wt $*$ sinpitch $*(\mathrm{ABS}($ zvalue $) * 1 \mathrm{E}-03)$

term3 $3 \mathrm{i}]=$ pot. $\mathrm{wt} *$ cospitch*xvalue*1E-03

$\mathrm{fs}[0, \mathrm{i}]=\mathrm{f}[0]$-shearerr*(1)-(pot.wt)* $\operatorname{sinpitch}^{*}(1) \quad ; *(1)$ refers to the cosax

$\mathrm{fs}[1, \mathrm{i}]=\mathrm{f}[1]$

$\mathrm{fs}[2, \mathrm{i}]=\mathrm{f}[2]-(-1.6 \mathrm{E}-03 * \mathrm{SQR}(\operatorname{pos}[2, \mathrm{i}])+3 \cdot 2 \mathrm{E}-03 * \operatorname{pos}[2, \mathrm{i}])-($ pot.wt $) * \cos p i t c h$

$\mathrm{fs}[3, \mathrm{i}]=\mathrm{f}[3]$

$\mathrm{fs}[4, \mathrm{i}]=\mathrm{f}[4]$-shearerr*(1)*(0.02652+(ABS(zvalue)*1E-03))-

(pot.wt)*sinpitch*(ABS(zvalue)*1E-03)+(pot.wt)*(cospitch*((xvalue)*1E-03)

$\mathrm{fs}[5, \mathrm{i}]=\mathrm{f}[5]$

IF LATCHED(1) GOTO 35

$\operatorname{tml}[i]=\operatorname{TIMER}(1)$

$\mathrm{i}=\mathrm{i}+1$

WAIT.EVENT , 5E-03

\section{END}

35 ext.total $=\mathrm{i}$

TYPE "Final outbound $\mathrm{i}=$ ", $\mathrm{i}$

; $\quad$ FOR $\mathrm{i}=1 \mathrm{TO}$ ext.total-1

; $\quad$ TYPE /F9.5, pos[2,i], shearerr[i], term1[i], term2[i], term3[i], f[4,i], fs[4,i]

\section{; END}

HERE loc.trip

DECOMPOSE tp []$=$ loc.trip

$\operatorname{pos}[0]=\operatorname{tp}[0]$

$\operatorname{pos}[1]=\operatorname{tp}[2]$

$\operatorname{pos}[2]=-(180-\operatorname{tp}[4])$

$\operatorname{pos}[3]=\operatorname{tp}[5]$

shearerr $=(-1 \mathrm{E}-05 * \operatorname{pos}[2] * \mathrm{SQR}(\operatorname{pos}[2])-4 \mathrm{E}-05 * \operatorname{SQR}(\operatorname{pos}[2])+0.1746 * \operatorname{pos}[2])$ 


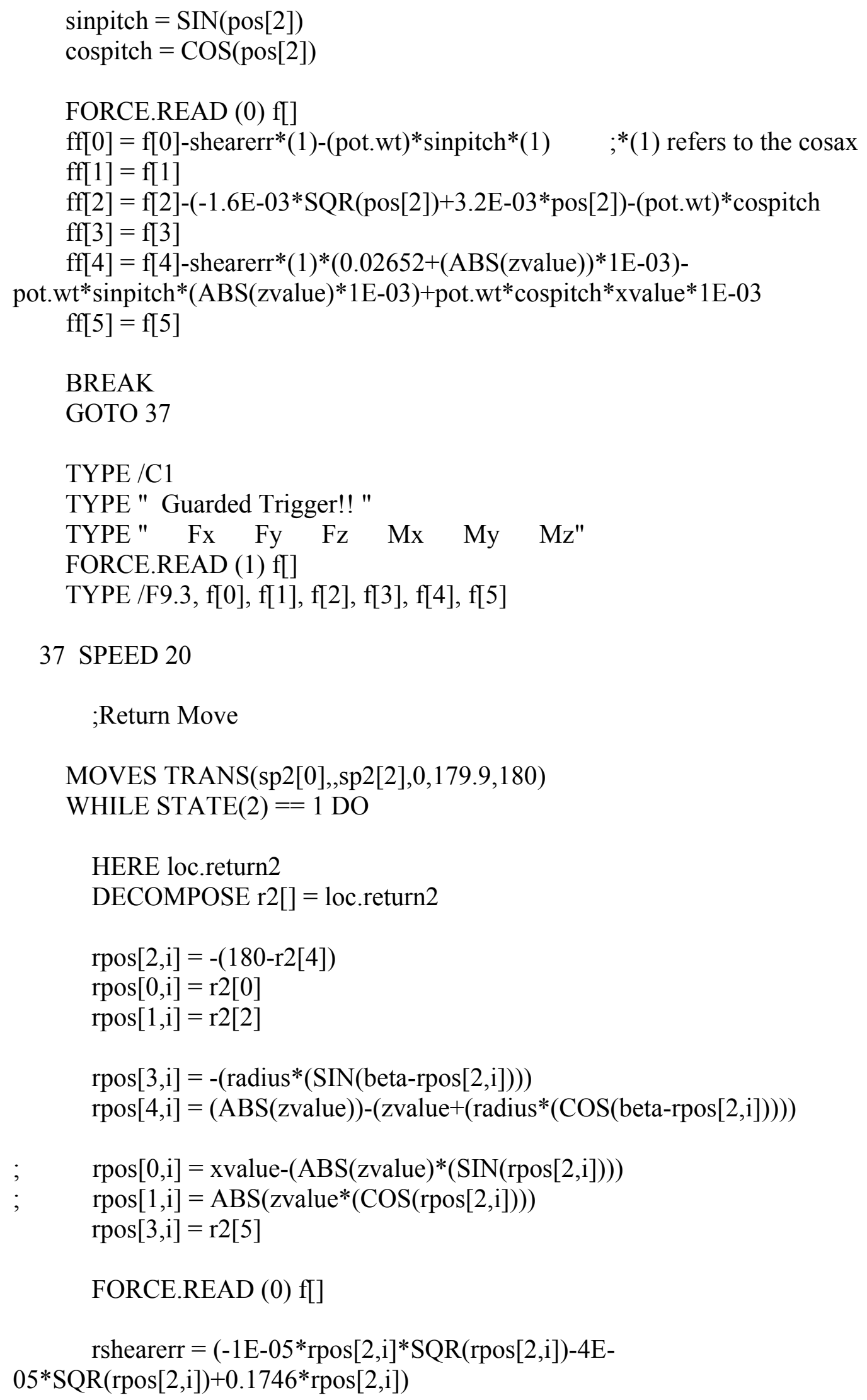


IF (flexdegree $<0$ ) THEN

TYPE "Wow:", flexdegree, "in flexion."

PROMPT "Are you sure?(y/n):", \$ans

IF \$ans $==$ "n" GOTO 42

END

TYPE /C3

43 PROMPT "How many degrees of EXTENSION are in your sweep?", extdegree

IF (extdegree $>10)$ THEN

TYPE "Wow:", extdegree, "in extension."

PROMPT "Are you sure?(y/n):", \$ans

IF \$ans == "n" GOTO 43

END

IF (extdegree < 0) THEN

TYPE "Wow:", extdegree, "in extension."

PROMPT "Are you sure?(y/n):", \$ans

IF \$ans == "n" GOTO 43

END

CALL spine_preload23()

;should I incorporate the change in position due to the preload?

HERE start.point. 1

DECOMPOSE $\mathrm{sp}[]=$ start.point.1

xstart $=\mathrm{sp}[0]$

$\mathrm{zstart}=\mathrm{sp}[2]$

MOVE TRANS(sp[0]+xvalue,,sp[2]+zvalue,0,180,180)

MOVE TRANS(sp[0]+xvalue,,sp[2]+zvalue,0,179.9,180)

BREAK

FORCE.MODE (-1)

FORCE.MODE (-1)

FORCE.MODE $(1)^{\wedge} \mathrm{H} 430$, for.trip, ${ }^{\wedge} \mathrm{H} 530$, mom.trip $\quad$;guarded for 3D

resultant foces and $\mathrm{X}-\mathrm{Z}$ plane moments

CALL fs.buf.config(-1, $-1,30,10$, stt $)$

IF $\mathrm{stt}<0$ GOTO 100

$\mathrm{i}=1$

CALL fs.buf.enable()

TIMER $(1)=0$ 
NOFLIP

MOVES TRANS(sp[0]+xvalue,,sp[2]+zvalue,0,180-extdegree,180)

WHILE STATE(2) $==1$ DO

HERE loc.start3

DECOMPOSE x3[] = loc.start3

$\operatorname{pos}[2, \mathrm{i}]=(180-\mathrm{x} 3[4])$

$\operatorname{pos}[0, \mathrm{i}]=$ xvalue $-(\mathrm{ABS}(\mathrm{zvalue}) *(\operatorname{SIN}(\operatorname{pos}[2, \mathrm{i}])))$

$\operatorname{pos}[1, \mathrm{i}]=\operatorname{ABS}(z v a l u e *(\operatorname{COS}(\operatorname{pos}[2, \mathrm{i}])))$

$; \operatorname{pos}[3, \mathrm{i}]=\mathrm{x} 3[5]$

FORCE.READ (0) f[]

shearerr $=\left(-1 \mathrm{E}-05^{*} \operatorname{pos}[2, \mathrm{i}] * \mathrm{SQR}(\operatorname{pos}[2, \mathrm{i}])-4 \mathrm{E}-\right.$

$05 * \operatorname{SQR}(\operatorname{pos}[2, \mathrm{i}])+0.1746 * \operatorname{pos}[2, \mathrm{i}])$

sinpitch $=\operatorname{SIN}(\operatorname{pos}[2, \mathrm{i}])$

$\operatorname{cospitch}=\operatorname{COS}(\operatorname{pos}[2, \mathrm{i}])$

$\mathrm{fs}[0, \mathrm{i}]=\mathrm{f}[0]$-shearerr*(1)-10* $\operatorname{sinpitch}^{*}(1) \quad ; *(1)$ refers to the cosax

$\mathrm{fs}[2, \mathrm{i}]=\mathrm{f}[2]-(-1.6 \mathrm{E}-03 * \mathrm{SQR}(\operatorname{pos}[2, \mathrm{i}])+3.2 \mathrm{E}-03 * \operatorname{pos}[2, \mathrm{i}])-10 * \operatorname{cospitch}$

$\mathrm{fs}[4, \mathrm{i}]=\mathrm{f}[4]$-shearerr $*(1) * 0.02652$

$\mathrm{fs}[1, \mathrm{i}]=\mathrm{f}[1]$

$\mathrm{fs}[3, \mathrm{i}]=\mathrm{f}[3]$

$\mathrm{fs}[5, \mathrm{i}]=\mathrm{f}[5]$

IF LATCHED(1) GOTO 44

$\mathrm{i}=\mathrm{i}+1 \quad$;highest value of $\mathrm{i}$ may not correspond to last calculated variables

WAIT.EVENT , 0.1

END

out.total $=\mathrm{i}$

TYPE "Final outbound $\mathrm{i}=$ ", $\mathrm{i}$

GOTO 45

44 TYPE /C1

TYPE " Guarded Trigger!! "

TYPE " Fx Fy Fz Mx My Mz"

FORCE.READ (1) f[]

TYPE /F9.3, f[0], f[1], f[2], f[3], f[4], f[5]

45

MOVES TRANS(sp[0]+xvalue,,sp[2]+zvalue,0,178,180);2 degrees on each side of the vertical seems to give a smooth transition response 
WHILE STATE(2)==1 DO ;will this loop run for all three moves? !!!!

HERE loc.start3

DECOMPOSE $\mathrm{x} 3[]=$ loc.start3

$$
\begin{aligned}
\operatorname{pos}[2, \mathrm{i}] & =(180-\mathrm{x} 3[4]) \\
\operatorname{pos}[0, \mathrm{i}] & =\mathrm{xvalue}-(\mathrm{ABS}(\mathrm{zvalue}) *(\operatorname{SIN}(\operatorname{pos}[2, \mathrm{i}]))) \\
\operatorname{pos}[1, \mathrm{i}] & =\mathrm{ABS}(\mathrm{zvalue}(\operatorname{COS}(\operatorname{pos}[2, \mathrm{i}]))) \\
; \operatorname{pos}[3, \mathrm{i}] & =\mathrm{x} 3[5]
\end{aligned}
$$

FORCE.READ (0) f[]

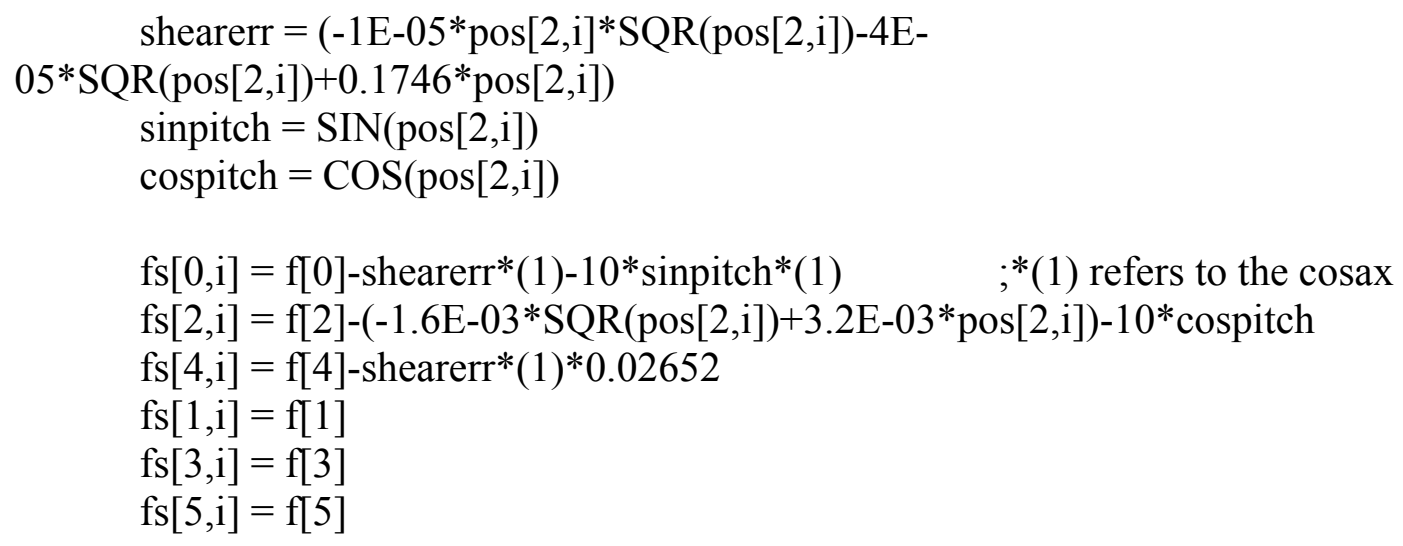

IF LATCHED(1) GOTO 46 variables

$\mathrm{i}=\mathrm{i}+1 \quad$;highest value of $\mathrm{i}$ may not correspond to last calculated

END
swe1 1 total $=i$

FLIP

MOVE TRANS(sp[0]+xvalue,,sp[2]+zvalue, 180,178,0)

MOVES TRANS(sp[0]+xvalue,,sp[2]+zvalue, 180,180-flexdegree,0)

WHILE STATE(2)==1 DO ;(no)will this loop run for all three moves? !!!!

HERE loc.start3

DECOMPOSE x3[] = loc.start3

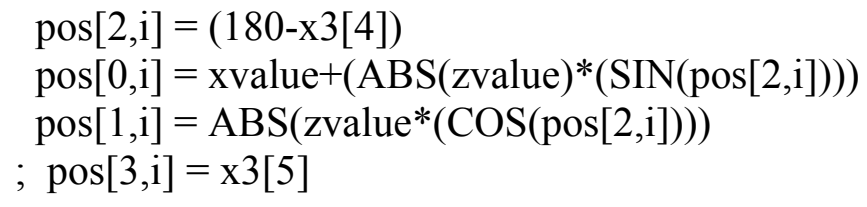

FORCE.READ (0) f[] 


$$
\text { shearerr }=(-1 \mathrm{E}-05 * \operatorname{pos}[2, \mathrm{i}] * \mathrm{SQR}(\operatorname{pos}[2, \mathrm{i}])-4 \mathrm{E}-
$$

$\left.05^{*} \operatorname{SQR}(\operatorname{pos}[2, \mathrm{i}])+0.1746^{*} \operatorname{pos}[2, \mathrm{i}]\right)$

sinpitch $=\operatorname{SIN}(\operatorname{pos}[2, \mathrm{i}])$

cospitch $=\operatorname{COS}(\operatorname{pos}[2, \mathrm{i}])$

$\mathrm{fs}[0, \mathrm{i}]=\mathrm{f}[0]$-shearerr*(1)-10*sinpitch*(1) $\quad ; *(1)$ refers to the cosax

$\mathrm{fs}[2, \mathrm{i}]=\mathrm{f}[2]-(-1.6 \mathrm{E}-03 * \mathrm{SQR}(\operatorname{pos}[2, \mathrm{i}])+3.2 \mathrm{E}-03 * \operatorname{pos}[2, \mathrm{i}])-10 * \operatorname{cospitch}$

$\mathrm{fs}[4, \mathrm{i}]=\mathrm{f}[4]$-shearerr* $(1) * 0.02652$

$\mathrm{fs}[1, \mathrm{i}]=\mathrm{f}[1]$

$\mathrm{fs}[3, \mathrm{i}]=\mathrm{f}[3]$

$\mathrm{fs}[5, \mathrm{i}]=\mathrm{f}[5]$

IF LATCHED(1) GOTO 46

$\mathrm{i}=\mathrm{i}+1 \quad$;highest value of $\mathrm{i}$ may not correspond to last calculated variables

WAIT.EVENT, 0.1

\section{END}

swe2.total $=\mathrm{i}$

TYPE "Final sweep $\mathrm{i}=$ =", $\mathrm{i}$

GOTO 48

46 TYPE /C1

TYPE " Guarded Trigger!! "

TYPE " Fx Fy $\mathrm{Fz}$ Mx $\mathrm{My}$ Mz"

FORCE.READ (1) f[]

TYPE /F9.3, f[0], f[1], f[2], f[3], f[4], f[5]

48

;Return Move

MOVES TRANS(sp[0]+xvalue,,sp[2]+zvalue,180,179.9,0)

WHILE STATE(2) $==1$ DO

HERE loc.return3

DECOMPOSE r3[] = loc.return3

$\operatorname{rpos}[2, \mathrm{i}]=(180-\mathrm{r} 3[4])$

$\operatorname{rpos}[0, i]=x v a l u e+($ ABS $(z v a l u e) *(\operatorname{SIN}(\operatorname{rpos}[2, \mathrm{i}])))$

$\operatorname{rpos}[1, \mathrm{i}]=\mathrm{ABS}\left(\right.$ zvalue $\left.^{*}(\operatorname{COS}(\operatorname{rpos}[2, \mathrm{i}]))\right)$

$; \operatorname{rpos}[3, \mathrm{i}]=\mathrm{r} 3[5]$

FORCE.READ (0) f[] 
rshearerr $=(-1 \mathrm{E}-05 * \operatorname{rpos}[2, \mathrm{i}] * \mathrm{SQR}(\mathrm{rpos}[2, \mathrm{i}])-4 \mathrm{E}-$

$05 * \operatorname{SQR}(\operatorname{rpos}[2, \mathrm{i}])+0.1746 * \operatorname{rpos}[2, \mathrm{i}])$

rsinpitch $=\operatorname{SIN}(\operatorname{rpos}[2, \mathrm{i}])$

rcospitch $=\operatorname{COS}(\operatorname{rpos}[2, \mathrm{i}])$

$\operatorname{rfs}[0, \mathrm{i}]=\mathrm{f}[0]$-rshearerr* $(1)-10 *$ rsinpitch $*(1) \quad ; *(1)$ refers to the cosax $\operatorname{rfs}[2, \mathrm{i}]=\mathrm{f}[2]-(-1.6 \mathrm{E}-03 * \operatorname{SQR}(\operatorname{rpos}[2, \mathrm{i}])+3.2 \mathrm{E}-03 * \operatorname{rpos}[2, \mathrm{i}])-10 *$ rcospitch $\operatorname{rfs}[4, \mathrm{i}]=\mathrm{f}[4]$-rshearerr* $(1) * 0.02652$

IF LATCHED(1) GOTO 55

$\mathrm{i}=\mathrm{i}+1$

WAIT.EVENT , 0.1

END

BREAK

GOTO 56

55 TYPE /C1

TYPE " Guarded Trigger!! "

TYPE " Fx Fy $\mathrm{Fz}$ Mx $\mathrm{My}$ Mz"

FORCE.READ (1) f[]

TYPE /F9.3, f[0], f[1], f[2], f[3], f[4], f[5]

NOFLIP

MOVES TRANS(sp[0]+xvalue,,sp[2]+zvalue, $0,179.9,180)$

56

70

SELECT FORCE $=2$

CALL fs.buf.read $\left(2,{ }^{\wedge} \mathrm{H} 3 \mathrm{~F},,,, \mathrm{p}[]\right.$, stt $)$

IF $\mathrm{stt}<0$ GOTO 100

CALL fs.buf.read( $0,{ }^{\wedge} \mathrm{H} 3 \mathrm{~F},,,, \mathrm{f}[]$, stt $)$

IF $\mathrm{stt}<0$ GOTO 100

CALL fs.buf.read $\left(1,{ }^{\wedge} \mathrm{H} 3 \mathrm{~F},,,,, \mathrm{j}[]\right.$, stt $)$

IF stt $<0$ GOTO 100

BREAK

SELECT FORCE $=1$

CALL write_to_file23() 
100 IF stt $<0$ THEN

TYPE " Error occured : ", \$ERROR(stt)

PAUSE

END

110 PROMPT "Would you like to perform another test?(y/n): ", \$ans

IF (\$ans $<>$ "y") AND (\$ans $<>$ "n") GOTO 110

IF \$ans $==$ "y" GOTO 3

IF \$ans == "n" GOTO 115

115 TYPE /C50, /U42

TYPE "----------------Ya'll come back now, ya her!-------------------"

TYPE /C5

\section{B.2 Spine_Mount23}

; ABSTRACT: Spine_mount2 is a modified version of Brian's spine.mount that will be used in the single

;segment axis of rotation study. The purpose of this program is to prepare the specimen for a $10 \mathrm{~N}$ preload

;application, resulting in the specimen carrying total pot weight.

; INPUT PARMS:

;

; OUTPUT PARMS:

;

; SIDE EFFECTS:

;

; DATA STRUCT:

;

; MISC:

;* Copyright (c) 1998 by ${ }^{34}$

AUTO REAL f[6]

AUTO \$ans, first.loc

\$ans $="$ "

SELECT FORCE $=1$

TYPE /C50, /U42 
TYPE "

TYPE "

FORCE.READ (0) f[]

TYPE /C2

TYPE " The current load cell readings are:"

TYPE " Fx Fy $\mathrm{Fz}$ Mx My Mz"

TYPE /F8.2, f[0], f[1], f[2], f[3], f[4], f[5]

TYPE /C2

10 PROMPT "Prepare specimen and when ready to continue, press'y': ", \$ans

IF \$ans $<>$ "y" THEN

GOTO 10

END

DETACH (0)

TYPE /C50,/U42

TYPE "

$* * * * * * * * * * * * * * * * * * * * * * * * * * * * * * * * * * * * * * * * * * * * * * * * * * * * * * * * * * * * * * * * * * * * * * * 11$

TYPE "

SAFETY WARNING!"

TYPE"

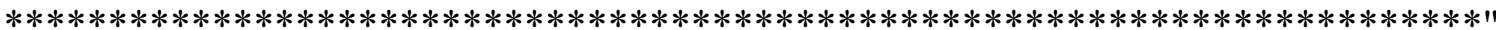

TYPE " "

TYPE " BE SURE TO DISABLE HIGH POWER WHEN WORKING

UNDER TEST FRAME!"

TYPE " "

TYPE "

$* * * * * * * * * * * * * * * * * * * * * * * * * * * * * * * * * * * * * * * * * * * * * * * * * * * * * * * * * * * * * * * * * * * * * * * * 1$

TYPE /C2

20 TYPE " Turn off high power and Insert spine."

PROMPT " Then restore high power and press 'y' when ready: ", \$ans

IF \$ans $<$ "y" GOTO 20

IF STATE(1) $==2$ THEN

TYPE " "

TYPE "You have not restored robot high power!!"

GOTO 20

END

ATTACH (0)

HERE first.loc

DECOMPOSE pos[] = first.loc

FLIP

MOVE TRANS(pos[0],pos[1],pos[2],180,179.9,0) ～;get rotary axes correct 


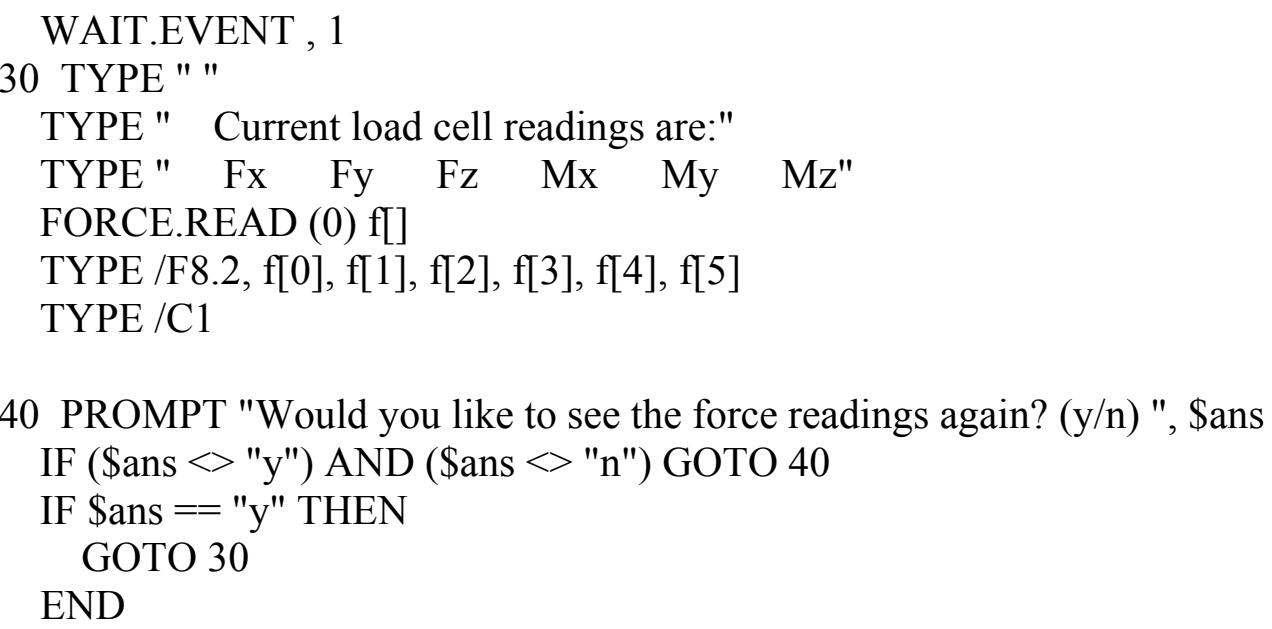

50 PROMPT "Would you like to zero in the x direction? (y/n)", \$ans

IF (\$ans <> "y") AND (\$ans <> "n") GOTO 50

IF $\$$ ans $==$ "y" GOTO 80

60 PROMPT "Would you like to apply pot weight preload plus 10 N? (y/n)", \$ans

IF (\$ans <> "y") AND (\$ans <> "n") GOTO 60

IF \$ans == "y" GOTO 70

IF \$ans == "n" GOTO 100

; Apply correct pot weight preload in $\mathrm{z}$ direction

70 ACCEL (3) 20, 20

FORCE.READ (0) f[]

IF (f[2] > -10) THEN ;spine is in tension

FORCE.MODE (1) ${ }^{\wedge} \mathrm{H} 212,-11$

DRIVE 3, 5, 3 ;slowly move jt3 5mm down until trip condition from above BREAK

END

$$
\text { ; FORCE.MODE (-1) }
$$

IF ( $\mathrm{f}[2]<-12)$ THEN ;spine is in compression

FORCE.MODE (1) ${ }^{\wedge} \mathrm{H} 12,-11$

DRIVE 3, $-5,3 \quad$;slowly move jt3 up until tensile force of $-0.5 \mathrm{~N}$ is met (TRIPPED)

BREAK

; FORCE.MODE (-1)

END ;if

IF LATCHED(1) THEN

TYPE /C1

TYPE " GUARDED TRIGGER!! Pot Weight preload reached" 
$\begin{array}{lllllll}\text { TYPE " Fx } & \text { Fy } & \mathrm{Fz} & \mathrm{Mx} & \mathrm{My} & \mathrm{Mz}\end{array}$

FORCE.READ (1) f[]

TYPE /F8.2, f[0], f[1], f[2], f[3], f[4], f[5]

TYPE " "

END ;if

FORCE.MODE (-1)

GOTO 30

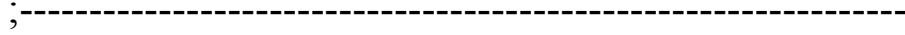 \\ ; Code to zero in $\mathrm{x}$ direction \\ 80 FORCE.READ (0) f[] \\ IF $(\mathrm{f}[0]<0)$ THEN \\ FORCE.MODE $(1)^{\wedge} \mathrm{H} 10,0$ \\ ACCEL (2) 100, 100 \\ DRIVE $1,-5,5$ \\ BREAK \\ FORCE.MODE (-1) \\ ELSE \\ FORCE.MODE (1)^ $\mathrm{H} 210,0$ \\ ACCEL (2) 100, 100 \\ DRIVE $1,5,5$ \\ BREAK \\ FORCE.MODE (-1) \\ END \\ IF LATCHED(1) THEN \\ TYPE /C1 \\ TYPE " GUARDED TRIGGER! Forces zeroed in x direction:" \\ TYPE " Fx Fy $\mathrm{Fz}$ Mx $\mathrm{My}$ Mz" \\ FORCE.READ (1) f[] \\ TYPE /F8.2, f[0], f[1], f[2], f[3], f[4], f[5] \\ TYPE " " \\ END \\ FORCE.MODE (-1)^H10, 0 \\ WAIT.EVENT, 0.5 \\ GOTO 30 \\ 100 TYPE " "
}

\title{
B.3 Spine_Preload23
}

; ABSTRACT: This program applies a $10 \mathrm{~N}$ compressive preload 


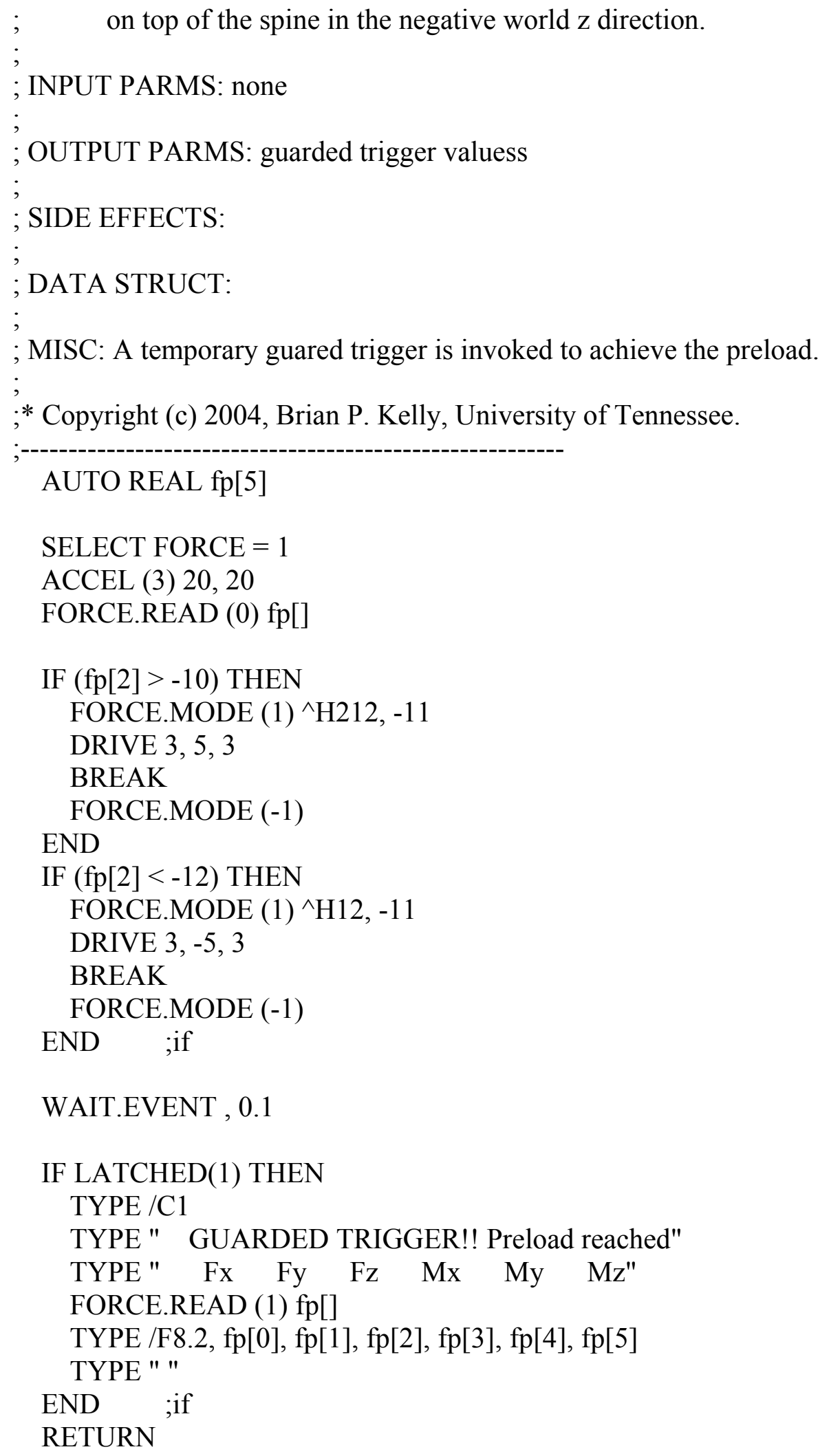




\section{B.4 Write to_File23}

; ABSTRACT: This program opens a file in the nfs directory ; of biomech8, writes user specified data to the file, closes ; the file and checks for errors through this process. The ; parent of this file resides in E: $\backslash$ Adept $\backslash$ Disks $\backslash$ Disk_C $\backslash$

; This program usually called to store data from force buffer.

; October 2003, by Brian P. Kelly, University of Tennessee.

;modified September 2004, by Henry Bonin, UTHSC

; INPUT PARMS: value of 'stt', user specified array variables.

; OUTPUT PARMS: Values of variable in .txt file.

; GLOBAL flex.total, ext.total, out.total, swe.total

\footnotetext{
ATTACH (dlun, 4) "NFS"

IF IOSTAT(dlun) $<0$ THEN

TYPE "Error attaching nfs"

GOTO 100

END ;if

\$file.name1 = "testresult.fl"

\$file.name2 = "testresult.ext"

\$file.name3 = "testresult.sweep"

\$file.name4 = "BaseJR3Data"

; $\quad$ flexion.total $=$ flex

; $\quad$ extension.total $=\mathrm{ext}$

; $\quad$ outbound.total $=$ out

; $\quad$ sweep.total $=$ sweep

IF \$test.ans == "f" GOTO 10

IF \$test.ans == "e" GOTO 20

IF \$test.ans == "s" GOTO 30

$; * * * * * * * * * * * * * * * * * * * * * * * *$ File 1 Test Result $* * * * * * * * * * * * * * * * * * * * * * * * * * *$
$;$ Flexion
$\quad 10$

TYPE "flex.total = ", flex.total

FOPENW (dlun) \$file.name1

IF IOSTAT(dlun) $<0$ THEN
} 
TYPE "Error opening file in biomech 8."

GOTO 100

\section{END}

; TYPE /F9.3, fs[0,2], fs[2,2], fs[4,2], /S

WRITE (dlun) "junk,", "t,", "Fx,", "Fy,", "Fz,", "Mx,", "My,", "Mz,", "x,", "z,", "pitch,", "roll,"

FOR $\mathrm{j}=1$ TO flex.total-1

WRITE (dlun) /F9.3, tm1[j], fs[0,j], fs[1,j], fs[2,j], fs[3,j], fs[4,j], fs[5,j], /S

WRITE (dlun) /F12.6, pos[0,j], pos[1,j], pos[2,j], pos[3,j]

; WRITE (dlun) /F9.3, p[0,j], p[1,j], p[3,j], p[5,j] ; base load cell data is read out in File 2

IF IOSTAT(dlun) $<0$ THEN

TYPE "Error writing to Biomech8."

GOTO 100

END

\section{END}

WRITE (dlun) /F9.3, 0, ff[0], ff[1], ff[2], ff[3], ff[4], ff[5], /S

WRITE (dlun) /F12.6, pos[0], pos[1], pos[2], pos[3]

IF IOSTAT(dlun) $<0$ THEN

TYPE "Error writing to Biomech8."

GOTO 100

END

FOR $\mathrm{j}=$ flex.total TO $\mathrm{i}-1$

WRITE (dlun) /F9.3, tm1[j], rfs[0,j], rfs[1,j], rfs[2,j], rfs[3,j], rfs[4,j], rfs[5,j], /S

WRITE (dlun) /F12.6, rpos[0,j], rpos[1,j], rpos[2,j], $\operatorname{rpos}[3, \mathrm{j}]$

; WRITE (dlun) /F9.3, p[0,j], p[1,j], p[3,j], p[5,j]

IF IOSTAT(dlun) $<0$ THEN

TYPE "Error writing to Biomech8."

GOTO 100

END

END

FCLOSE (dlun)

IF IOSTAT(dlun) $<0$ THEN

TYPE "Error closing file1 "

GOTO 100

END

GOTO 110

;Extension

20

TYPE "ext.total = ", ext.total

FOPENW (dlun) \$file.name2 
IF IOSTAT(dlun) $<0$ THEN

TYPE "Error opening file in biomech 8."

GOTO 100

END

WRITE (dlun) "junk,", "t,", "Fx,", "Fy,", "Fz,", "Mx,", "My,", "Mz,", "x,", "z,", "pitch,", "roll,"

FOR $\mathrm{j}=1$ TO ext.total-1

WRITE (dlun) /F9.3, tml[j], fs[0,j], fs[1,j], fs[2,j], fs[3,j], fs[4,j], fs[5,j], /S

WRITE (dlun) /F12.6, pos[0,j], pos[1,j], pos[2,j], pos[3,j]

; WRITE (dlun) /F9.3, p[0,j], p[1,j], p[3,j], p[5,j]

IF IOSTAT(dlun) $<0$ THEN

TYPE "Error writing to Biomech8."

GOTO 100

END

END

WRITE (dlun) /F9.3, 0, ff[0], ff[1], ff[2], ff[3], ff[4], ff[5], /S

WRITE (dlun) /F12.6, pos[0], pos[1], pos[2], pos[3]

IF IOSTAT(dlun) $<0$ THEN

TYPE "Error writing to Biomech8."

GOTO 100

END

FOR $\mathrm{j}=$ ext.total TO i-1

WRITE (dlun) /F9.3, tml[j], rfs[0,j], rfs[1,j], rfs[2,j], rfs[3,j], rfs[4,j], rfs[5,j], /S

WRITE (dlun) /F12.6, rpos[0,j], rpos[1,j], rpos[2,j], rpos[3,j]

; WRITE (dlun) /F9.3, p[0,j], p[1,j], p[3,j], p[5,j]

IF IOSTAT(dlun) $<0$ THEN

TYPE "Error writing to Biomech8."

GOTO 100

END

END

FCLOSE (dlun)

IF IOSTAT(dlun) $<0$ THEN

TYPE "Error closing file1 "

GOTO 100

END

GOTO 110

;Sweep

30

TYPE "out.total = ", out.total

TYPE "swe1.total = ", swe1.total

TYPE "swe2.total = ", swe2.total 
FOPENW (dlun) \$file.name3

IF IOSTAT(dlun) $<0$ THEN

TYPE "Error opening file in biomech 8."

GOTO 100

END

FOR $\mathrm{j}=1$ TO out.total-1

WRITE (dlun) /F9.3, fs[0,j], fs[1,j], fs[2,j], fs[3,j], fs[4,j], fs[5,j], /S

WRITE (dlun) /F12.6, pos[0,j], pos[1,j], pos[2,j]

IF IOSTAT(dlun) $<0$ THEN

TYPE "Error writing to Biomech8."

GOTO 100

END

END

FOR $\mathrm{j}=$ out.total TO swe 1. total -1

WRITE (dlun) /F9.3, fs[0,j], fs[1,j], fs[2,j], fs[3,j], fs[4,j], fs[5,j], /S

WRITE (dlun)/F12.6, pos[0,j], pos[1,j], pos[2,j]

IF IOSTAT(dlun) $<0$ THEN

TYPE "Error writing to Biomech8."

GOTO 100

END

END

FOR $\mathrm{j}=$ swe1.total TO swe 2.total-1

WRITE (dlun) /F9.3, fs[0,j], fs[1,j], fs[2,j], fs[3,j], fs[4,j], fs[5,j], /S

WRITE (dlun)/F12.6, pos[0,j], pos[1,j], pos[2,j]

IF IOSTAT(dlun) $<0$ THEN

TYPE "Error writing to Biomech8."

GOTO 100

END

END

FOR $\mathrm{j}=$ swe 2.total TO $\mathrm{i}-1$

WRITE (dlun) /F9.3, rfs[0,j], rfs[1,j], rfs[2,j], rfs[3,j], rfs[4,j], rfs[5,j], /S

WRITE (dlun) /F12.6, rpos[0,j], $\operatorname{rpos}[1, \mathrm{j}], \operatorname{rpos}[2, \mathrm{j}]$

IF IOSTAT(dlun) $<0$ THEN

TYPE "Error writing to Biomech8."

GOTO 100

END

END

FCLOSE (dlun)

IF IOSTAT(dlun) $<0$ THEN

TYPE "Error closing file1 "

GOTO 100

END 
FOPENW (dlun) \$file.name4

IF IOSTAT(dlun) $<0$ THEN

TYPE "Error opening file in biomech 8."

GOTO 100

END $\quad$;if

FOR $n=0$ TO stt-1

WRITE (dlun) /F9.3, p[0,n], p[1,n], p[2,n], p[3,n], p[4,n], p[5,n], p[6,n], /S

WRITE (dlun) /F9.3, f[1,n], f[2,n], f[3,n], f[4,n], f[5,n], f[6,n], /S

WRITE (dlun) /F9.3, j[1,n], j[2,n], j[3,n], j[4,n], j[5,n], j[6,n]

IF IOSTAT(dlun) $<0$ THEN

TYPE "Error writing to Biomech8."

GOTO 100

END

END

FCLOSE (dlun)

IF IOSTAT(dlun) $<0$ THEN

TYPE "Error closing file2 "

GOTO 100

END

100 TYPE \$ERROR(IOSTAT(dlun))

RETURN 
Appendix C: Tabulated Data 
Table C.1 Harvested Spine Condition Flexion Data.

\begin{tabular}{|c|c|c|c|c|c|c|c|c|c|c|}
\hline \multicolumn{11}{|c|}{ Points Along Disc Midline } \\
\hline \multicolumn{11}{|c|}{ A1 } \\
\hline Spine & $\mathbf{F x}$ & $\mathbf{F y}$ & $\overline{\mathbf{F z}}$ & $\mathbf{M x}$ & My & $\mathbf{M z}$ & $\bar{x}$ & $\bar{z}$ & pitch & roll \\
\hline 0105416 L3 -L4 & 12.6 & 20.6 & 245.5 & -1.9 & -7.5 & 0.9 & 24.4 & -38.2 & 3.3 & 0.0 \\
\hline UJ00D35 L3-L4 & 44.9 & -21.1 & 204.9 & -0.2 & -7.6 & -0.6 & 28.7 & -63.2 & 4.1 & 0.0 \\
\hline ADS0612051 L5-S1 & 18.7 & 1.4 & 123.1 & 0.0 & -7.8 & 0.1 & -5.3 & -43.6 & 4.7 & 0.0 \\
\hline ADS0612051 L3-L4 & 22.8 & 3.7 & 202.9 & 1.0 & -7.6 & 0.1 & -24.7 & -68.5 & 3.9 & 0.0 \\
\hline DRT060069 L3-L4 & 44.4 & 1.7 & 217.1 & 0.1 & -7.7 & 0.0 & -25.2 & -73.6 & 3.5 & 0.0 \\
\hline DRT050786 L3-L4 & -3.1 & -2.9 & 249.4 & 0.9 & -7.7 & 0.0 & 13.4 & -41.0 & 4.0 & 0.0 \\
\hline UF01A030 L3-L4 & -15.6 & -44.3 & 259.8 & -0.2 & -7.6 & -0.9 & -34.8 & -76.9 & 3.8 & 0.0 \\
\hline Mean & 17.8 & -5.8 & 214.7 & 0.0 & -7.6 & -0.1 & -3.4 & -57.9 & 3.9 & $\overline{0.0}$ \\
\hline Standard Deviation & 22.5 & 20.9 & 46.2 & 1.0 & 0.1 & 0.6 & 25.8 & 16.4 & 0.4 & 0.0 \\
\hline \multicolumn{11}{|c|}{ C1 } \\
\hline Spine & $\mathbf{F x}$ & $\mathbf{F y}$ & $\mathbf{F z}$ & $\mathbf{M x}$ & My & $\mathbf{M z}$ & $\mathbf{x}$ & $\begin{array}{l}\mathbf{z} \\
-\end{array}$ & pitch & roll \\
\hline \multicolumn{11}{|l|}{0105416 L3 -L4 } \\
\hline UJ00D35 L3-L4 & 9.0 & -35.5 & 143.5 & 0.4 & -7.9 & -0.6 & 18.1 & -68.0 & 5.5 & 0.0 \\
\hline ADS0612051 L5-S1 & 26.8 & 1.4 & 159.4 & -0.1 & -8.0 & 0.3 & 6.4 & -48.5 & 6.9 & 0.0 \\
\hline ADS0612051 L3-L4 & 25.2 & -33.6 & 156.9 & 1.0 & -7.7 & -0.7 & -35.2 & -65.4 & 5.4 & 0.0 \\
\hline DRT060069 L3-L4 & 62.2 & -0.3 & 214.3 & 0.1 & -7.8 & 0.0 & -34.1 & -72.0 & 5.1 & 0.0 \\
\hline DRT050786 L3-L4 & -37.3 & -2.7 & 68.8 & 0.7 & -8.0 & -0.2 & 5.9 & -38.3 & 5.1 & 0.0 \\
\hline UF01 A030 L3-L4 & -39.9 & -47.1 & 205.7 & 0.8 & -7.9 & -0.5 & -45.1 & -74.7 & 6.1 & 0.0 \\
\hline Mean & 7.7 & -19 & 158.1 & 0.5 & -7.9 & -0.3 & -14.0 & -61.2 & 5.7 & $\overline{0.0}$ \\
\hline Standard Deviation & 39.8 & 21.5 & 52.2 & 0.4 & 0.1 & 0.4 & 27.1 & 14.5 & 0.7 & 0.0 \\
\hline \multicolumn{11}{|c|}{ P1 } \\
\hline Spine & $\mathbf{F x}$ & Fy & $\overline{F z}$ & Mx & $\mathbf{M y}$ & $\mathbf{M z}$ & $\bar{x}$ & $\overline{\mathbf{z}}$ & pitch & roll \\
\hline 0105416 L3 -L4 & -22.4 & 20.4 & -148.7 & -0.9 & -7.8 & 1.6 & 6.0 & -33.2 & 6.2 & $\overline{0.0}$ \\
\hline UJ00D35 L3-L4 & -57.0 & -44.2 & -214.2 & 1.4 & -7.8 & 0.7 & 8.8 & -64.0 & 5.9 & 0.0 \\
\hline ADS0612051 L5-S1 & -93.2 & -5.2 & -33.9 & 0.6 & -8.0 & -0.2 & -18.9 & -32.8 & 8.8 & 0.0 \\
\hline ADS0612051 L3-L4 & -26.8 & -102.0 & -140.0 & 0.6 & -7.9 & -0.3 & -45.4 & -63.1 & 5.8 & 0.0 \\
\hline DRT060069 L3-L4 & -53.4 & 1.0 & -453.5 & 0.0 & -7.9 & 0.0 & -43.0 & -70.9 & 4.6 & 0.0 \\
\hline DRT050786 L3-L4 & -118.8 & 12.8 & -403.6 & 0.0 & -8.1 & -0.4 & -2.4 & -35.7 & 4.4 & 0.0 \\
\hline UF01A030 L3-L4 & -86.5 & -63.0 & -367.1 & 1.8 & -7.8 & 0.3 & -54.7 & -72.9 & 5.9 & 0.0 \\
\hline Mean & -65.4 & -25.7 & -251.6 & 0.5 & -7.9 & 0.2 & -21.4 & -53.2 & 6.0 & $\overline{0.0}$ \\
\hline Standard Deviation & 35.6 & 45.3 & 157.6 & 0.9 & 0.1 & 0.7 & 26.4 & 18.4 & 1.5 & 0.0 \\
\hline
\end{tabular}

\begin{tabular}{|c|c|c|c|c|c|c|c|c|c|c|}
\hline \multicolumn{11}{|c|}{ Points in Subjacent Body } \\
\hline \multicolumn{11}{|c|}{$\overline{A 2}$} \\
\hline Spine & $\mathbf{F x}$ & Fy & $\mathbf{F z}$ & $\mathbf{M x}$ & My & $\mathbf{M z}$ & $\mathbf{x}$ & $\bar{z}$ & pitch & roll \\
\hline 0105416 L3 -L4 & -12.1 & 20.2 & 231.5 & -2.1 & -7.4 & 0.7 & 20.3 & -48.9 & 3.5 & 0.0 \\
\hline UJ00D35 L3-L4 & -20.9 & -20.6 & 145.2 & 0.5 & -7.7 & -0.5 & 23.3 & -85.9 & 4.1 & 0.0 \\
\hline ADS0612051 L5-S1 & -54.7 & 0.9 & 68.3 & 0.5 & -8.0 & -0.3 & -16.4 & -49.3 & 5.8 & 0.0 \\
\hline ADS0612051 L3-L4 & -10.2 & -6.2 & 185.5 & 1.1 & -7.5 & -0.1 & -25.7 & -78.4 & 3.8 & 0.0 \\
\hline DRT060069 L3-L4 & -15.4 & -4.0 & 207.4 & 0.3 & -7.7 & -0.1 & -26.0 & -83.1 & 3.6 & 0.0 \\
\hline DRT050786 L3-L4 & -41.4 & -5.0 & 204.1 & 0.8 & -7.8 & 0.1 & 12.9 & -50.0 & 3.6 & 0.0 \\
\hline UF01A030 L3-L4 & -41.4 & -42.9 & 224.9 & 0.5 & -7.7 & -0.9 & -35.6 & -88.4 & 3.4 & 0.0 \\
\hline Mean & -28.0 & -8.2 & 181.0 & 0.2 & -7.7 & -0.2 & -6.7 & -69.2 & 4.0 & 0.0 \\
\hline Standard Deviation & 17.6 & 19.5 & 57.3 & 1.1 & 0.2 & 0.5 & 24.8 & 18.7 & 0.8 & 0.0 \\
\hline \multicolumn{11}{|c|}{$\mathbf{C 2}$} \\
\hline Spine & $\mathbf{F x}$ & Fy & $\mathbf{F z}$ & $\mathbf{M x}$ & My & $\mathbf{M z}$ & $\mathbf{x}$ & $\mathbf{z}$ & pitch & roll \\
\hline 0105416 L3 -L4 & -40.6 & 21.3 & 199.9 & -2.0 & -7.6 & 0.9 & 11.2 & -46.4 & 4.9 & 0.0 \\
\hline UJ00D35 L3-L4 & -91.5 & -28.8 & 1.8 & 1.6 & -7.8 & 0.2 & 13.4 & -82.2 & 5.3 & 0.0 \\
\hline ADS0612051 L5-S1 & -100.2 & 2.4 & -19.0 & 1.0 & -8.0 & -0.8 & -20.0 & -44.2 & 6.9 & 0.0 \\
\hline ADS0612051 L3-L4 & -58.7 & -31.0 & 86.5 & 1.7 & -7.7 & -0.5 & -36.1 & -76.0 & 5.5 & 0.0 \\
\hline DRT060069 L3-L4 & -48.3 & -6.2 & 139.5 & 0.3 & -7.9 & -0.1 & -35.7 & -81.6 & 5.5 & 0.0 \\
\hline DRT050786 L3-L4 & -110.9 & -2.1 & 10.5 & 0.7 & -8.0 & 0.1 & 4.6 & -47.5 & 4.6 & 0.0 \\
\hline UF01A030 L3-L4 & -100.2 & -45.5 & 130.2 & 1.5 & -7.8 & -0.4 & -45.7 & -85.6 & 5.5 & 0.0 \\
\hline Mean & -78.6 & -12.9 & 78.5 & 0.7 & -7.8 & -0.1 & -15.5 & -66.2 & 5.5 & $\overline{0.0}$ \\
\hline Standard Deviation & 28.6 & 23.1 & 82.9 & 1.3 & 0.1 & 0.6 & 24.9 & 19.1 & 0.7 & 0.0 \\
\hline \multicolumn{11}{|c|}{$\overline{\mathbf{P 2}}$} \\
\hline Spine & $\overline{F x}$ & Fy & $\overline{F z}$ & Mx & My & $\mathbf{M z}$ & $\bar{x}$ & $\bar{z}$ & pitch & roll \\
\hline 0105416 L3 -L4 & -111.6 & 22.3 & -241.4 & -0.8 & -7.8 & 1.6 & 3.8 & -43.8 & 5.4 & 0.0 \\
\hline UJ00D35 L3-L4 & -127.2 & -39.8 & -308.2 & 1.2 & -7.7 & 1.7 & 3.5 & -75.8 & 4.7 & 0.0 \\
\hline ADS0612051 L5-S1 & -201.6 & -2.0 & -276.5 & 1.9 & -7.5 & -2.1 & -27.6 & -39.5 & 6.2 & 0.0 \\
\hline ADS0612051 L3-L4 & -85.5 & -91.5 & -176.6 & 1.6 & -7.8 & -0.3 & -45.1 & -72.5 & 5.5 & 0.0 \\
\hline DRT060069 L3-L4 & -145.9 & -1.2 & -513.9 & 0.0 & -7.7 & 0.0 & -44.5 & -80.6 & 3.5 & 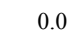 \\
\hline DRT050786 L3-L4 & -171.7 & 8.6 & -361.7 & 0.1 & -8.1 & -0.2 & -3.4 & -44.7 & 3.9 & 0.0 \\
\hline UF01A030 L3-L4 & -146.9 & -63.5 & -312.9 & 2.6 & -7.6 & 0.0 & -55.2 & -83.4 & 5.2 & 0.0 \\
\hline Mean & -141.5 & -23.9 & -313.0 & 0.9 & -7.8 & 0.1 & -24.1 & -62.9 & 4.9 & 0. \\
\hline Standard Deviation & 38.3 & 42.0 & 106.3 & 1.2 & 0.2 & 1.3 & 25.2 & 19.3 & 0.9 & 0.0 \\
\hline
\end{tabular}


Table C.2 Harvested Spine Condition Extension Data.

\begin{tabular}{|c|c|c|c|c|c|c|c|c|c|c|}
\hline \multicolumn{11}{|c|}{ Points Along Disc Midline } \\
\hline \multicolumn{11}{|c|}{ A1 } \\
\hline Spine & $\mathbf{F x}$ & $\mathbf{F y}$ & $\mathbf{F z}$ & Mx & My & $\mathbf{M z}$ & $\mathbf{x}$ & $\mathbf{z}$ & pitch & roll \\
\hline 0105416 L3 -L4 & -56.7 & 5.8 & -270.5 & 0.3 & 7.8 & 1.7 & 24.6 & -38.0 & -1.9 & 180.0 \\
\hline UJ00D35 L3-L4 & -118.9 & -28.9 & -276.6 & -2.6 & 7.7 & -0.6 & 29.0 & -63.3 & -1.8 & 180.0 \\
\hline ADS0612051 L5-S1 & -170.5 & -14.7 & -232.9 & 0.3 & 8.0 & -0.1 & -4.9 & -43.6 & -2.5 & 180.0 \\
\hline ADS0612051 L3-L4 & -16.9 & -89.9 & -183.3 & 0.9 & 7.9 & -1.5 & -24.4 & -68.6 & -2.5 & 180.0 \\
\hline DRT060069 L3-L4 & -117.7 & -2.8 & -371.8 & -0.5 & 8.1 & 0.0 & -24.9 & -73.5 & -1.5 & 180.0 \\
\hline DRT050786 L3-L4 & -51.7 & 2.7 & -255.7 & -0.3 & 7.5 & -2.9 & 13.7 & -40.9 & -2.9 & 180.0 \\
\hline UF01A030 L3-L4 & -54.1 & -59.1 & -253.6 & 1.6 & 7.8 & -1.2 & -34.5 & -76.9 & -2.1 & 180.0 \\
\hline Mean & -83.8 & -26.7 & -263.5 & $\overline{0.0}$ & 7.8 & -0.7 & -3.0 & -57.8 & -2.2 & 180.0 \\
\hline Standard Deviation & 53.3 & 35.8 & 57.0 & 1.3 & 0.2 & 1.4 & 25.8 & 16.5 & 0.5 & 0.0 \\
\hline \multicolumn{11}{|c|}{ C1 } \\
\hline Spine & $\mathbf{F x}$ & $\mathbf{F y}$ & $\mathbf{F z}$ & $\mathbf{M x}$ & My & $\mathbf{M z}$ & $\mathbf{x}$ & $\mathrm{z}$ & pitch & roll \\
\hline 0105416 L3 -L4 & -32.8 & 10.3 & -205.0 & 0.4 & 7.8 & 1.6 & 13.6 & -37.5 & $\begin{array}{l}-3.3 \\
\end{array}$ & 180.0 \\
\hline UJ00D35 L3-L4 & -122.5 & -20.7 & -316.1 & -3.2 & 7.3 & -0.4 & 18.6 & -68.0 & -3.3 & 180.0 \\
\hline ADS0612051 L5-S1 & -201.0 & -21.3 & -246.9 & -0.4 & 8.0 & 0.6 & -11.9 & -38.7 & -3.8 & 180.0 \\
\hline ADS0612051 L3-L4 & 1.5 & -66.0 & -121.7 & 0.8 & 8.0 & -0.3 & -34.7 & -65.4 & -3.3 & 180.0 \\
\hline DRT060069 L3-L4 & -109.7 & -1.9 & -398.8 & -0.9 & 7.7 & 0.0 & -33.7 & -71.9 & -3.0 & 180.0 \\
\hline DRT050786 L3-L4 & -3.2 & -5.4 & -79.9 & -0.4 & 7.4 & -3.0 & 6.2 & -38.2 & -3.6 & 180.0 \\
\hline UF01A030 L3-L4 & -29.4 & -61.1 & -103.4 & 1.3 & 7.9 & -0.5 & -44.6 & -74.8 & -2.9 & 180.0 \\
\hline Mean & -71.0 & -23.7 & -210.3 & -0.3 & 7.8 & -0.3 & -12.3 & -56.4 & -3.3 & 180.0 \\
\hline Standard Deviation & 75.4 & 29.4 & 118.6 & 1.4 & 0.3 & 1.4 & 25.7 & 17.3 & 0.3 & 0.0 \\
\hline \multicolumn{11}{|c|}{ P1 } \\
\hline Spine & $\mathbf{F x}$ & $\mathbf{F y}$ & $\mathbf{F z}$ & $\mathbf{M x}$ & My & $\mathbf{M z}$ & $\mathbf{x}$ & $\mathbf{z}$ & pitch & roll \\
\hline 0105416 L3 -L4 & -15.5 & 12.8 & -32.1 & -0.1 & 7.9 & 1.4 & 6.3 & -33.1 & -4.0 & 180.0 \\
\hline UJ00D35 L3-L4 & -105.0 & -12.4 & -224.6 & -2.5 & 7.6 & -0.2 & 9.0 & -64.3 & -4.8 & 180.0 \\
\hline ADS0612051 L5-S1 & -160.5 & -18.7 & -173.8 & -0.8 & 7.9 & 1.3 & -18.4 & -33.0 & -5.2 & 180.0 \\
\hline ADS0612051 L3-L4 & 37.5 & -35.0 & 62.1 & 0.7 & 8.0 & 0.8 & -45.2 & -63.3 & -3.4 & 180.0 \\
\hline DRT060069 L3-L4 & -31.7 & -3.7 & -83.9 & -1.2 & 8.0 & 0.0 & -42.7 & -70.9 & -4.1 & 180.0 \\
\hline DRT050786 L3-L4 & 58.0 & -7.9 & 137.1 & -0.3 & 7.7 & -2.1 & -2.4 & -35.8 & -3.8 & 180.0 \\
\hline UF01A030 L3-L4 & 13.4 & -58.7 & 103.0 & 1.0 & 7.9 & 0.4 & -54.6 & -73.1 & -3.0 & 180.0 \\
\hline Mean & -29.1 & -17.6 & -30.3 & -0.4 & 7.9 & 0.2 & -21.1 & -53.4 & -4.0 & 180.0 \\
\hline Standard Deviation & 78.6 & 23.2 & 138.8 & 1.2 & 0.1 & 1.2 & 26.4 & 18.5 & 0.8 & 0.0 \\
\hline
\end{tabular}

\begin{tabular}{|c|c|c|c|c|c|c|c|c|c|c|}
\hline \multicolumn{11}{|c|}{ Points in Subjacent Body } \\
\hline \multicolumn{11}{|c|}{$\overline{\mathrm{A2}}$} \\
\hline Spine & $\mathbf{F x}$ & Fy & $\mathbf{F z}$ & $\mathbf{M x}$ & My & $\mathbf{M z}$ & $\mathbf{x}$ & $\mathbf{z}$ & pitch & roll \\
\hline 0105416 L3 -L4 & -26.5 & 6.3 & -243.5 & 0.2 & 7.9 & 1.5 & 20.3 & -48.9 & -2.1 & 180.0 \\
\hline UJ00D35 L3-L4 & -74.2 & -22.8 & -280.7 & -2.8 & 7.6 & -0.6 & 23.4 & -86.0 & -2.4 & 180.0 \\
\hline ADS0612051 L5-S1 & -103.7 & 1.7 & -148.2 & 0.1 & 8.0 & 0.9 & -16.5 & -49.4 & -4.5 & 180.0 \\
\hline ADS0612051 L3-L4 & -10.7 & -86.5 & -166.3 & 1.8 & 7.7 & -1.4 & -25.8 & -78.5 & -2.6 & 180.0 \\
\hline DRT060069 L3-L4 & -61.9 & -2.0 & -339.2 & -0.6 & 8.1 & 0.0 & -26.1 & -83.1 & -1.7 & 180.0 \\
\hline DRT050786 L3-L4 & 9.5 & 4.1 & -170.0 & -0.4 & 7.6 & -2.7 & 12.8 & -49.8 & -2.8 & 180.0 \\
\hline UF01A030 L3-L4 & 6.8 & -49.2 & -197.1 & 2.0 & 7.7 & -0.7 & -35.7 & -88.4 & -2.0 & 180.0 \\
\hline Mean & -37.2 & -21.2 & -220.7 & $\overline{0.0}$ & 7.8 & -0.4 & -6.8 & -69.2 & -2.6 & 180.0 \\
\hline Standard Deviation & 43.5 & 35.0 & 70.1 & 1.6 & 0.2 & 1.4 & 24.8 & 18.7 & 0.9 & 0.0 \\
\hline \multicolumn{11}{|c|}{$\overline{\mathrm{C} 2}$} \\
\hline Spine & $\mathbf{F x}$ & Fy & $\overline{F z}$ & $\begin{array}{l}\mathbf{M x} \\
\end{array}$ & My & $\mathbf{M z}$ & $\bar{x}$ & $\bar{z}$ & pitch & roll \\
\hline 0105416 L3 -L4 & 26.8 & 9.9 & -178.0 & 0.1 & 7.9 & 1.5 & 11.0 & -46.4 & -3.4 & 180.0 \\
\hline UJ00D35 L3-L4 & -53.0 & -10.3 & -235.2 & -2.8 & 7.5 & -0.2 & 13.3 & -82.5 & -3.7 & 180.0 \\
\hline ADS0612051 L5- & -80.1 & 5.8 & -103.5 & -0.2 & 8.0 & 0.9 & -19.9 & -44.4 & -5.3 & 180.0 \\
\hline ADS0612051 L3-L4 & 19.1 & -59.1 & -109.3 & 1.4 & 7.9 & -0.2 & -36.1 & -76.1 & -3.2 & 180.0 \\
\hline DRT060069 L3-L4 & -21.9 & -2.3 & -271.7 & -1.0 & 8.0 & 0.0 & -35.6 & -81.5 & -3.1 & 180.0 \\
\hline DRT050786 L3-L4 & 54.9 & 5.6 & -63.9 & -0.3 & 7.6 & -2.6 & 4.6 & -47.4 & -3.5 & 180.0 \\
\hline UF01A030 L3-L4 & 29.7 & -49.2 & -121.1 & 2.0 & 7.8 & 0.1 & -45.6 & -85.6 & -2.8 & 180.0 \\
\hline Mean & -3.5 & -14.2 & -154.7 & -0.1 & 7.8 & -0.1 & -15.5 & -66.3 & -3.6 & 180.0 \\
\hline Standard Deviation & 49.3 & 28.2 & 76.1 & 1.6 & 0.2 & 1.3 & 24.8 & 19.1 & 0.8 & 0.0 \\
\hline \multicolumn{11}{|c|}{$\mathbf{P 2}$} \\
\hline Spine & $\mathbf{F x}$ & $\mathbf{F y}$ & $\mathbf{F z}$ & $\mathbf{M x}$ & My & $\mathbf{M z}$ & $\bar{x}$ & $\bar{z}$ & pitch & roll \\
\hline 0105416 L3 -L4 & 54. & 15.2 & 10.0 & -0.5 & 7.9 & 1.2 & 3.4 & -44.0 & -3.9 & 180. \\
\hline UJ00D35 L3-L4 & 30.4 & -4.2 & -28.1 & -1.0 & 8.0 & -0.1 & 3.2 & -76.1 & -5.4 & 180.0 \\
\hline ADS0612051 L5- & -5.9 & 9.4 & -5.3 & -0.3 & 7.9 & 0.8 & -28.0 & -39.7 & -6.2 & 180.0 \\
\hline ADS0612051 L3-L & 57.4 & -28.1 & 109.4 & 0.9 & 8.0 & 0.6 & -45.5 & -72.6 & -3.2 & 180. \\
\hline DRT060069 L3-L4 & 75.8 & 0.7 & 19.0 & -1.2 & 7.9 & -0.1 & -44.5 & -80.6 & -3.9 & 180.0 \\
\hline DRT050786 L3-L4 & 103.1 & -0.4 & 135.8 & -0.2 & 7.7 & -2.2 & -3.8 & -44.8 & -3.5 & 180.0 \\
\hline UF01A030 L3-L4 & 79.5 & -50.3 & 108.0 & 1.5 & 7.9 & 0.8 & -55.5 & -83.7 & -2.9 & 180.0 \\
\hline Mean & 56.3 & -8.3 & 49.8 & -0.1 & 7.9 & 0.1 & -24.4 & -63.1 & -4.1 & 180.0 \\
\hline Standard Deviation & 35.7 & 23.0 & 65.8 & 1.0 & 0.1 & 1.1 & 25.2 & 19.3 & 1.2 & 0.0 \\
\hline
\end{tabular}




\section{Vita}

Nephi Alan Zufelt was born in Moab, Utah, on April 14, 1980. He graduated from Grand County High School in 1998 and then went on to Southern Utah University (SUU) to study Computer Science. After one year he volunteered to serve a full time mission for his church in Sao Paulo, Brazil. He spent the next 2 years learning to appreciate the Brazilian culture and to love the people of Brazil. Upon returning, he realized that computer science was not a field that he had an interest in pursuing, which led him to transfer to Utah State University (USU) in Logan, Utah, to study Mechanical Engineering. Between transferring from SUU to USU, Nephi traveled to Atlanta, Georgia, for a job where he met his future wife Elizabeth Michelle Thomas.

Nephi graduated in May, 2006, with a Bachelor of Science degree in mechanical engineering. The following semester Nephi moved his family to Memphis, Tennessee, to pursue a Master of Science degree with a major in Biomedical Engineering. Under the guidance of Dr. Denis DiAngelo, Nephi studied spine biomechanics and the effects of implanting spinal devices on the human spine.

Nephi recently accepted a position as a Product Development Engineer with Choice Spine in Knoxville, Tennessee. Nephi spends most of his spare time playing with his daughter Zoe, but when he gets a chance he enjoys going on dates with his wife, playing sports (basketball, volleyball, soccer, etc.), hiking and camping. 